Aus der Klinik für Unfall-, Hand- und Wiederherstellungschirurgie

(Chefarzt: Dr. med. H.-J. Walde)

des Nordwest-Krankenhauses in Sanderbusch

\title{
Optimierung der Rotationsausrichtung der femoralen Implantatkomponente in der Kniegelenksendoprothetik mit bandspannungsbasierter Navigation
}

\author{
INAUGURAL-DISSERTATION \\ zur Erlangung des Doktorgrades \\ der Medizinischen Fakultät \\ der Georg-August-Universität zu Göttingen
}

vorgelegt von

Jens Joachim Bussert

aus Köln

Göttingen 2013 
Dekan: Prof. Dr. med. H. K. Kroemer

I. Berichterstatter: Priv.-Doz. Dr. med. T. A. Walde

II. Berichterstatter: Prof. Dr. med. H.-M. Klinger

III. Berichterstatter/in:

Tag der mündlichen Prüfung: 01.10.2013 


\section{Abkürzungsverzeichnis}

Abb.

a.p.

ARO

$B Q S$

bzgl.

bzw.

ca.

$\mathrm{Cl}$

$\mathrm{cm}$

DGOOC

d. h.

DIMDI

FIP

FRC

ICD-10

IRO

ISO

$M$

MAD

$\max$.

$\min$.

OP

$\mathrm{n}$

PE

PKA

ROM

SD

Tab.

TEA

TFG

TIP

u. a.

Val

Var

VRML
Abbildung

anterior-posterior

Außenrotation

Bundesgeschäftsstelle Qualitätssicherung $\mathrm{GmbH}$

bezüglich

beziehungsweise

circa

95\%-Konfidenzintervall

Zentimeter

Deutsche Gesellschaft für Orthopädie und Orthopädische Chirurgie das heißt

Deutsches Institut für Medizinische Dokumentation und Information

Femurdurchstoßpunkt

Hüftrotationszentrum

International Statistical Classification of Diseases and Related Health

Problems (Internationale statistische Klassifikation der Krankheiten und verwandter Gesundheitsprobleme), aktuell gültige Version ICD-10 Innenrotation

International Organization for Standardization (Internationale

Organisation für Normung)

Mittelwert

Mechanische Achsdeviation

maximal

minimal

Operation

Anzahl

Polyethylen

posteriore Kondylenachse

Range of Motion (Bewegungsumfang)

Standard Deviation (Standardabweichung)

Tabelle

transepikondyläre Achse

Tibiofemoralgelenk

Tibiadurchstoßpunkt

unter anderem

Valgus

Varus

Virtual Reality Modeling Language 


\section{Inhaltsverzeichnis}

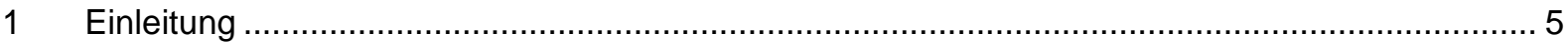

1.1 Aufbau und Biomechanik des gesunden Kniegelenks................................................... 5

1.1.1 Definition der menschlichen Beinachsen ........................................................... 10

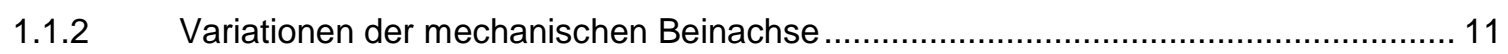

1.2 Gonarthrose - Die Arthrose im Kniegelenk ........................................................... 12

1.3 Hilfslinien zur Positionierung der Femurkomponente (knöcherne Landmarken) ................. 14

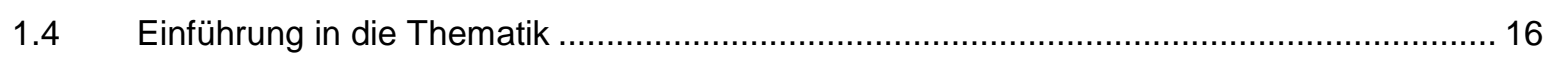

1.5 Die Bedeutung des Weichteilmanagements in der Kniegelenksendoprothetik ................... 18

1.6 Computergestützte Navigation in der modernen Kniegelenksendoprothetik....................... 19

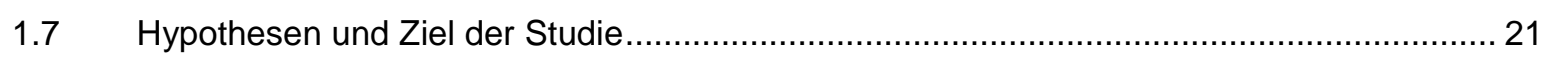

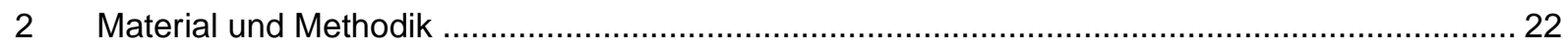

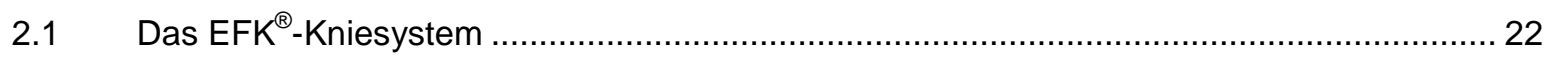

2.2 Die Operationstechnik mit dem Navigationssystem PiGalileo TKR Light ${ }^{\circledR}$, Version 1.3 ...... 23

2.2.1 Messungen am Femur zur Bestimmung der knöchernen Landmarken ...................... 26

2.2.2 Navigationsbasierte Festlegung der tibialen Resektion und Messung des

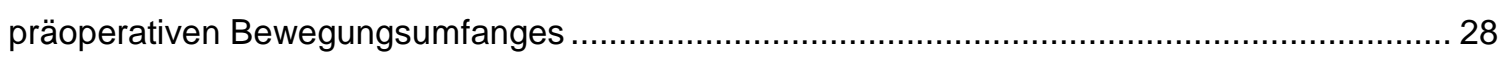

2.2.3 Bestimmung der Rotationsausrichtung der femoralen Implantatkomponente mit bandspannungsbasierter Navigation .............................................................................. 30

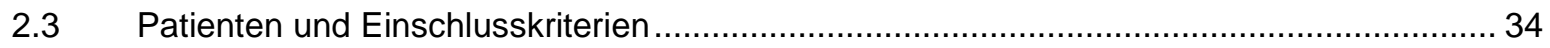

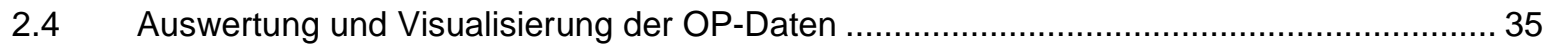

2.4.1 Erstellung von Funktionsdiagrammen in Abhängigkeit der knöchernen Landmarken 37

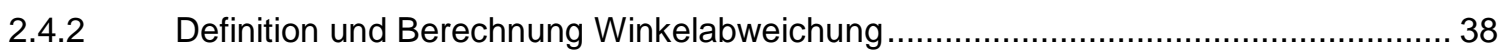

2.4.3 Bestimmung der femoralen Rotationsausrichtungen zur tibialen Resektionsfläche

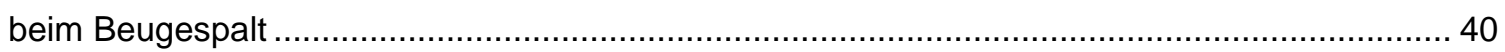

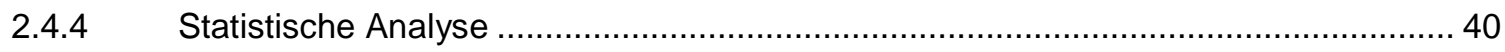

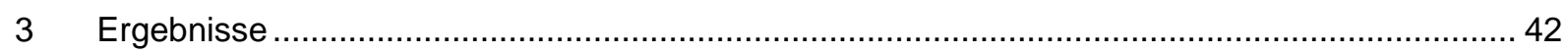

3.1 Winkelabweichung und Achsdeviation bei Auswertung der Funktionsdiagramme in

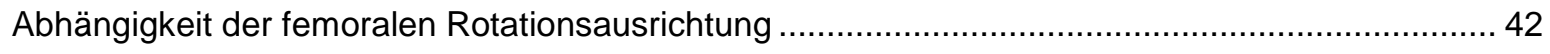

3.2 Femorale Rotationsausrichtung bei Analyse des Beugespaltes in Abhängigkeit der knöchernen Landmarken und mittels bandspannungsbasierter Navigation ................................... 52

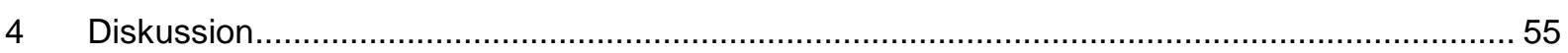

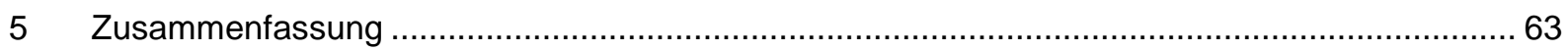

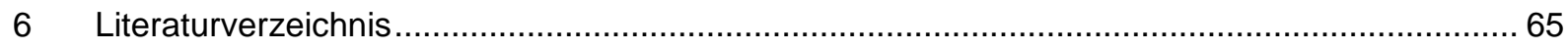

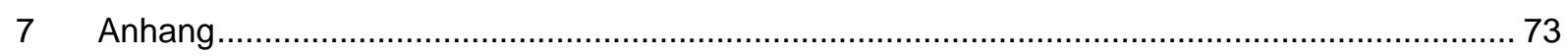

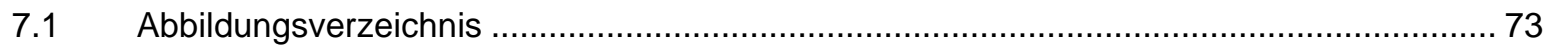

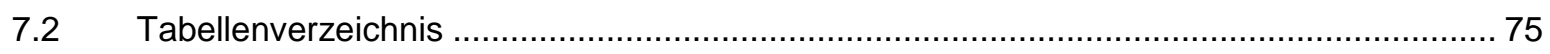




\section{$1 \quad$ Einleitung}

\subsection{Aufbau und Biomechanik des gesunden Kniegelenks}

Das Kniegelenk (Articulatio genus) stellt die bewegliche Verbindung zwischen Oberschenkelknochen (Femur), Schienbein (Tibia) und Kniescheibe (Patella) dar. Es ist das größte Gelenk des Menschen und gehört zu den am häufigsten verletzten Gelenken (Wirth et al. 2005).

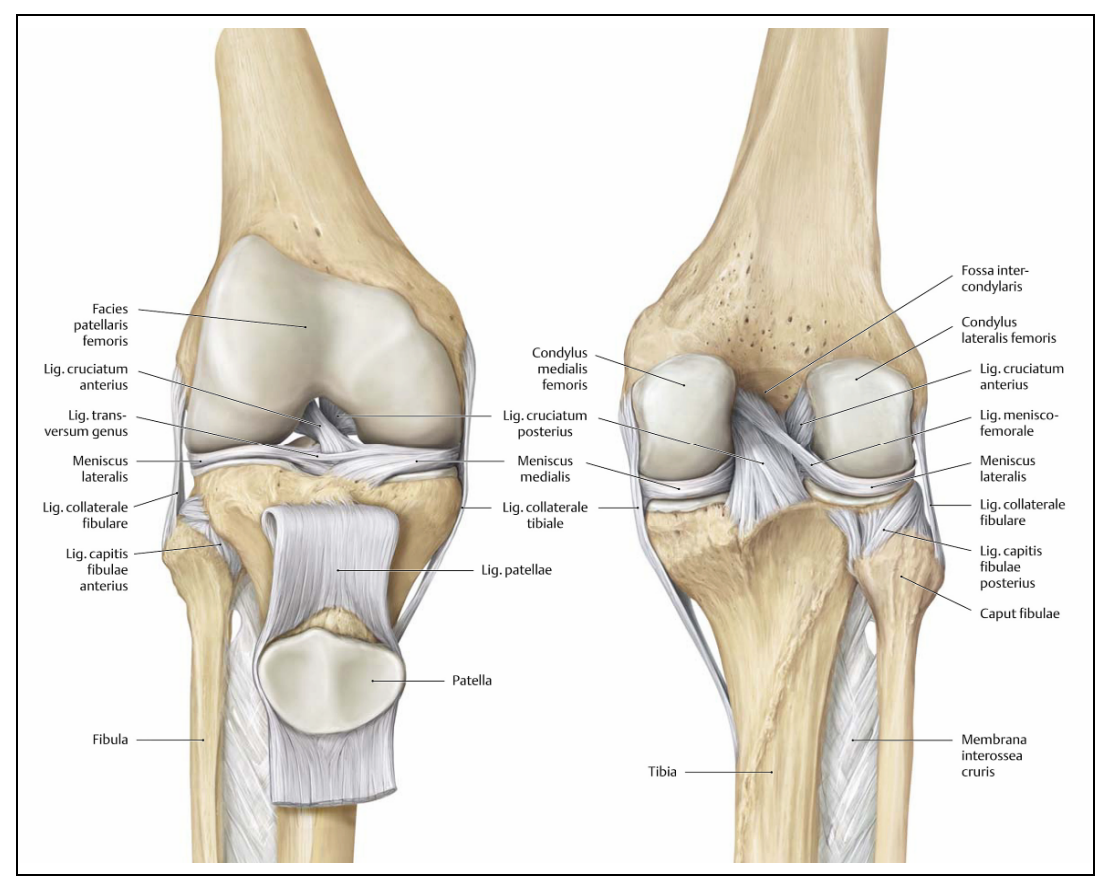

Abbildung 1: Anatomie des gesunden menschlichen Kniegelenks (aus Schünke et al. 2007, Seite 438, mit freundlicher Genehmigung des Georg-Thieme-Verlags)

Der Bewegungsablauf des Kniegelenks ist aufgrund seiner Anatomie (siehe Abb. 1) ein komplexer Vorgang. Das Kniegelenk ist ein zusammengesetztes Gelenk, das sich in zwei separate Gelenkanteile, das Tibiofemoralgelenk (bikondyläres Gelenk) und das Femoropatellargelenk, gliedern lässt (Benninghoff 1994). Im Femoropatellargelenk steht eine Rinne im distalen Oberschenkelknochen, dem so genannten Patellagleitlager, mit der in die Quadrizepssehne eingelagerten Patella in Verbindung. Die Patella ist das größte Sesambein des menschlichen Körpers und das Femoropatellargelenk der am stärksten belastete Teil des Kniegelenks in Beugung (Wirth et al. 2005). Zusätzlich lässt sich das Tibiofemoralgelenk (TFG) noch in ein mediales und laterales Kniegelenkskompartiment einteilen. 
Die Gelenkanteile von Femur und Tibia passen geometrisch nur unzureichend zusammen. In der Sagittalebene weisen physiologisch die Konturen der Femurkondylen eine mediolaterale Asymmetrie auf. Das Tibiaplateau, das um ca. $7^{\circ}-9^{\circ}$ nach dorsal abfällt, ist medial konkav und lateral konvex ausgebildet (Wirth et al. 2005). Insgesamt sind das mediale und das laterale Kompartiment des natürlichen Kniegelenks in der sagittalen Schnittebene zueinander versetzt, wobei das mediale weiter ventral und das laterale wenige Millimeter weiter dorsal lokalisiert ist. Die Inkongruenz der artikulierenden Gelenkflächen im TFG wird im gesunden Zustand durch die beiden C-förmig gebogenen Faserknorpelscheiben mit keilförmigem Querschnitt, den Menisci, sowohl lateral als auch medial vermindert (Wirth et al. 2005). Die Menisci beteiligen sich somit an der Gelenkstabilisation und sorgen für eine gleichmäßige Druckverteilung. Neben dem knöchernen Gerüst wird der komplexe Kapsel-Bandapparat als passiver Stabilisator bezeichnet. Zu den aktiven Stabilisatoren zählen die umgebenden Muskelgruppen mit ihren Sehnen (Wirth et al. 2005). Die entscheidende Stabilität im Kniegelenk wird erst durch die Gesamtheit aller aktiven und passiven Stabilisatoren erreicht. Das mediale und das laterale Seitenband (Ligamentum collaterale) haben ihren Ursprung am jeweiligen Epicondylus femoris und inserieren an der Tibia und am Fibulaköpfchen. Die Kollateralligamente stabilisieren das Knie gegenüber einem Varus- sowie Valgusstress und verhindern durch Anspannung in Streckung (Extension) eine Rotation der Tibia gegenüber dem Femur (Wagner und Schabus 1982). Bei zunehmender Kniebeugung erschlaffen diese Bänder und lassen dadurch eine definierte Außen- und Innenrotation zu. Ein Teil des medialen Kollateralbandsystems (Ligamenta meniscotibiale et meniscofemorale mediale) ist mit dem medialen Meniscus verwachsen (Benninghoff 1994). Das zusätzlich vorhandene Ligamentum collaterale mediale posterius zieht direkt durch einen Teil des medialen Meniscus und fixiert dadurch den Meniscus stärker zum Tibiakopf. Der laterale Meniscus hat keine strukturelle Verbindung zum Ligamentum collaterale laterale (Benninghoff 1994). Dieses zieht, von der Gelenkkapsel abgehoben, zum Caput fibulae. Ventral und dorsal strahlen Fasern des lateralen Meniscus in das vordere bzw. hintere Kreuzband ein (Ligamenta meniscofemoralia anterius et posterius). Im Zusammenspiel mit den Kreuzbändern bestimmen die Kollateralbänder die Beweglichkeit im Gelenk und sind hinsichtlich ihrer Bandspannung als Stabilitätskriterium nicht zu vernachlässigen (Wirth et al. 2005). 
Die beiden Kreuzbänder, das Ligamentum cruciatum anterius sowie das Ligamentum cruciatum posterius, ziehen innerhalb der interkondylären Notch (die zentrale Aussparung der Femurtrochlea) durch das Zentrum im Gelenkspalt zwischen Femur und Tibia (Wirth et al. 2005). Beide Bänder bestehen aus verschiedenen Bündeln und garantieren insbesondere bei der Beugung durch Limitierung der Translationsund Rotationsbewegungen eine wesentliche Stabilisierung des Kniegelenks (Benninghoff 1994). Unter Translation wird die Verschiebung des Femur gegenüber der Tibia nach anterior respektive posterior verstanden. Einer der wichtigsten passiven Kniegelenksstabilisatoren ist das vordere Kreuzband (Hollis et al. 1991; Noyes et al. 1992). Das Ligamentum cruciatum anterius verläuft in der Gelenkhöhle von superior, posterior und lateral nach inferior, anterior und medial. Dabei entspringt es an der inneren Fläche des lateralen Condylus femoris und befestigt sich medial im anterioren Bereich der Area intercondylaris der Tibia (Benninghoff 1994). Es besteht aus einem anteromedialen sowie posterolateralen und intermediären Bündel, die je nach Stellung des Kniegelenks einen unterschiedlichen Spannungszustand aufweisen. In Extension spannt sich das posterolaterale Bündel im Zusammenspiel mit dem hinteren Anteil des Ligamentum cruciatum posterius. Das anteromediale Bündel befindet sich hingegen in Beugung unter größerer Spannung. In fast allen Gelenkpositionen sind die verschiedenen Bündel der Kreuzbänder unterschiedlich zueinander angespannt (Benninghoff 1994). Bei der Flexion verhindert insbesondere das vordere Kreuzband, dass die Femurkondylen dorsal aus der Pfanne gleiten. Zudem nimmt es auch eine limitierende Rolle bei der axialen Rotation des Kniegelenks ein (Andersen und Dyhre-Poulsen 1997). Der Verlauf des Ligamentum cruciatum posterius ist entsprechend von medial nach lateral. Es hat seinen Ursprung an der Innenfläche des medialen Femurkondylus und zieht zur Insertion in die Area intercondylaris posterior tibiae. Das hintere Kreuzband stellt sich kräftiger dar und verhindert primär die posteriore Translation der Tibia (Whiteside 2004).

Der aktive physiologische Bewegungsumfang des gesunden menschlichen Kniegelenks, die range of motion (ROM), wird mit Hilfe der Neutral-Null-Methode ermittelt und kann bei gestrecktem Hüftgelenk von $0^{\circ}$ in Streckstellung bis ca. $125^{\circ}$ in Flexion betragen. Bei zusätzlicher Flexion im Hüftgelenk kann eine Kniebeugung von ungefähr $140^{\circ}$ erreicht werden (Wirth et al. 2005). 
Die passive Beugung wird durch die dorsalen Muskelgruppen des Ober- und Unterschenkels begrenzt und ist bis ca. 160 möglich (Benninghoff 1994). Die Extension endet in der Regel in der Neutral-Null-Stellung, jedoch kann das Kniegelenk passiv um $5^{\circ}-10^{\circ}$ überstreckt werden. Eine Rotation im TFG ist nur in gebeugtem Zustand unter geringster Spannung der Kreuzbänder möglich. Da sich die Kreuzbänder bei der Innenrotation umeinander wickeln, kann der Unterschenkel bis ca. 10 nach innen gegen den Oberschenkel rotiert werden. Während der Außenrotation ist aufgrund der fehlenden Hemmung durch die Kreuzbänder eine Rotation bis ungefähr 30을 durchführbar (Benninghoff 1994). Das Drehzentrum des Knies ist dadurch in die Kavität der medialen Tibiagelenkfläche verlagert. Eine mediale und laterale Aufklappbarkeit im Sinne einer Abduktion bzw. Adduktion ist beim gesunden Kniegelenk aufgrund des straffen Kollateralbandapparats und der gespannten hinteren Gelenkkapsel in Streckstellung fast gar nicht und in Beugung ab $20^{\circ}$ nur in sehr geringem Maße möglich.

Eine Beugung im Kniegelenk ist aus biomechanischer Perspektive ein komplizierter Prozess. Im Wesentlichen stellt die Knieflexion durch die geometrische Form der Femurkondylen und des Tibiaplateaus eine kombinierte Roll-Gleitbewegung dar (Müller 1982). Initial rollen die Femurkondylen bei einer Beugebewegung ausgehend von der Streckstellung (siehe Abb. 2). Erst ab einem Flexionswinkel von ca. $20^{\circ}-30^{\circ}$ gleiten die Gelenkflächen (Nägerl et al. 1993).

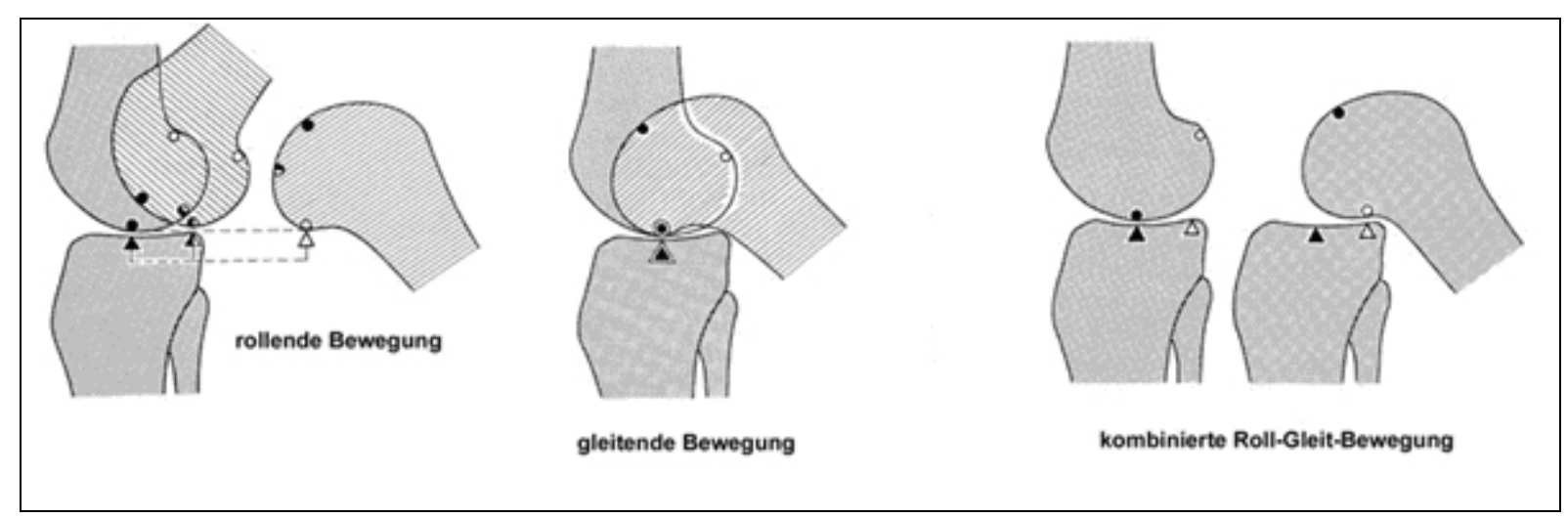

Abbildung 2: Roll-Gleitbewegung (modifiziert aus Müller 1982, Seite 7 und 8, mit freundlicher Genehmigung des Springer-Verlags) 
Durch die unterschiedliche anatomische Krümmung des medialen und lateralen Tibiaplateaus und die Position der Gelenkkompartimente wird die physiologische Rollbewegung (femoral roll back) mit anschließender Gleitbewegung bei zunehmender Flexion notwendig (Nägerl et al. 1993).

Aufgrund des Roll-Gleitmechanismus verkleinert sich auch die femorotibiale Kontaktfläche, wobei sich die femoralen Kontaktpunkte aus maximaler Extensionsposition heraus in unterschiedlichen Abständen (medial: $17 \mathrm{~mm}$ und lateral: $22 \mathrm{~mm}$ ) in die maximale Flexion nach dorsal bewegen (Pinskerova et al. 2004). Deswegen findet die Kniebeugung nicht um eine fixierte Drehachse statt. Bei Flexionswinkeln von $5^{\circ}-25^{\circ}$ liegt die momentane Drehachse in der Nähe des Gelenkspalts und wandert durch verstärkte Beugung in Richtung des Zentrums der Femurkondylen (Nägerl et al. 1993). Betrachtet man weiterhin das mediale und laterale Gelenkkompartiment aufgrund der unterschiedlichen Krümmungsradien ihrer Funktionsflächen sowie deren Verbindung als eine jeweilige Gelenkkette, so lassen sich diese so genannten dimeren Ketten miteinander koppeln und als ein Viergelenkgetriebe verstehen (Kubein-Meesenburg et al. 1991; Kubein-Meesenburg et al. 1993). Dadurch entsteht letztlich die natürliche Kinematik des Kniegelenks. Die ligamentären Strukturen nehmen hierbei eine untergeordnete Stellung ein (Nägerl et al. 1993). Beim Übergang von der Beugung in die Streckung wird während der Endphase der Extension ab ca. 10ำ zumeist eine Außenrotation der Tibia um $5^{\circ}-10^{\circ}$ hervorgerufen. Die Streckstellung verleiht dem Knie durch Anspannung der dorsalen Kapsel wieder den höchsten Stabilitätszustand.

Das Tibiofemoralgelenk und das Femoropatellargelenk sind von einer bindegewebigen Kapsel (Capsula articularis) umgeben. Diese besteht aus zwei Schichten: der stabilisierenden äußeren Faserschicht (Membrana fibrosa) und der innen liegenden Membrana synovialis (Wirth et al. 2005). Die Membrana synovialis produziert die wichtige Synovialflüssigkeit, die ihrerseits die Versorgung des hyalinen Gelenkknorpels gewährleistet. Zusätzliche Bedeutung hat sie in ihrer Funktion als Gleitmittel, weswegen sie auch "Gelenkschmiere" genannt wird. Eine stetige Be- und Entlastung und Bewegung des Kniegelenks ist für einen konstanten Austausch der Synovialflüssigkeit notwendig. 


\subsubsection{Definition der menschlichen Beinachsen}

Die Stellung und somit auch die Form des menschlichen Beins ist einerseits vom Collum-Corpus-Winkel (Collodiaphysenwinkel) des Femur und andererseits von der physiologischen Entwicklung des Kniegelenks abhängig. Die Längsachsen der Knochenschäfte von Femur und Tibia verlaufen in der Frontalebene nicht in einer Geraden, so dass sich die beiden Geraden in einem bestimmten Winkel schneiden. Die Femurschaftachse bildet mit der Horizontalen des Kniegelenks oder Kniebasislinie einen Winkel von ca. $81^{\circ} \pm 2^{\circ}$ und stellt die anatomische Achse des Oberschenkels dar. Die anatomische Tibiaschaftachse verläuft von der Mitte des Kniegelenks durch die Mitte des oberen Sprunggelenks und ist nahezu identisch mit der mechanischen Achse. Sie kreuzt die Kniebasislinie in einem Winkel von durchschnittlich $93^{\circ}$. Daraus resultiert ein nach lateral offener Abduktionswinkel von durchschnittlich $174^{\circ}$, der die anatomische Beinachse von $6^{\circ}$ Valgusstellung impliziert (siehe Abb. 3).

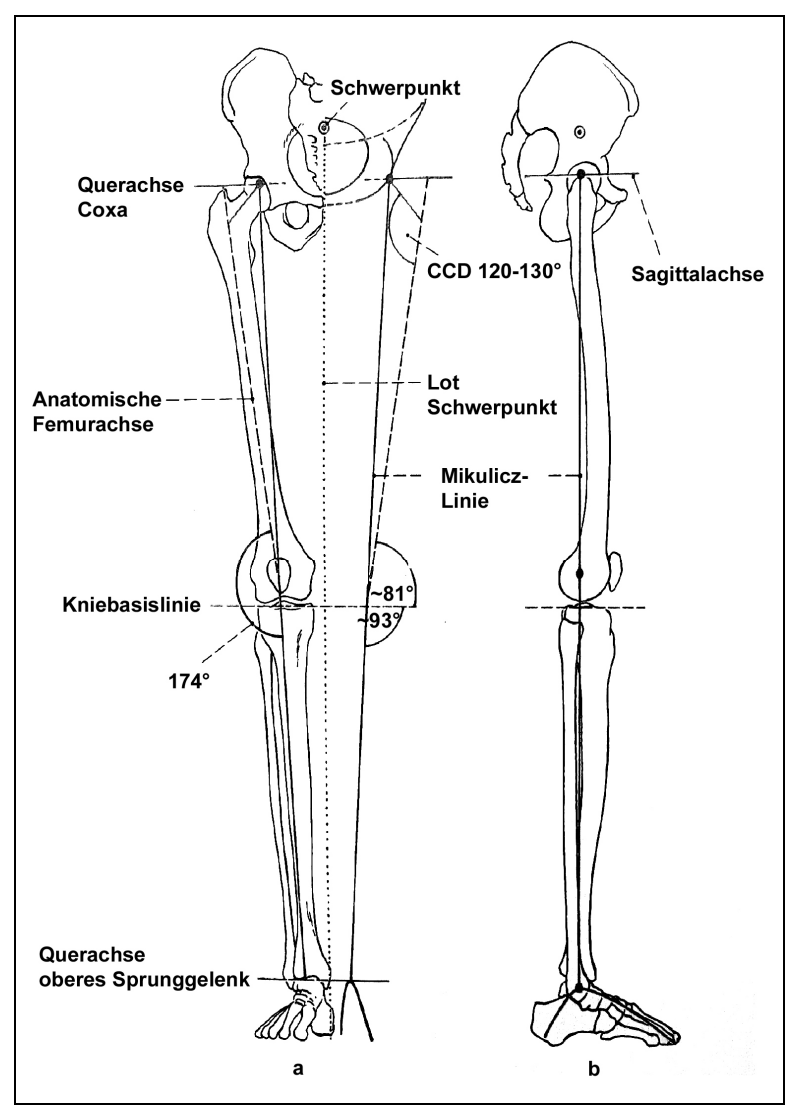

Abbildung 3: Schematische Darstellung der wichtigsten Beinachsen und Winkel in Extension, a: ventrale Ansicht; b: laterale Ansicht (aus Benninghoff 1994, Seite 370, mit freundlicher Genehmigung des Elsevier-Verlags) 
Die mechanische Achse des Femur verläuft physiologisch von der Mitte des Caput femoris durch das Zentrum des Kniegelenks bzw. nach Paley und Pfeil im Durchschnitt $4 \mathrm{~mm}( \pm 2 \mathrm{~mm})$ medial des Kniegelenkszentrums und bildet mit der anatomischen Achse der Tibia (die der mechanischen Tibiaachse entspricht) die Traglinie des Beins (Benninghoff 1994; Paley und Pfeil 2000). Klinisch wird diese Verbindungslinie als Mikulicz-Linie bezeichnet. Bei einem gesunden, normgerechten Kniegelenk stellt sie sich als Gerade aus den beiden mechanischen Achsen dar.

\subsubsection{Variationen der mechanischen Beinachse}

Durch den Verlauf der Traglinie in der Frontalebene werden beim Menschen drei unterschiedliche Beinstellungen festgelegt. Im Idealfall verläuft die Traglinie (mechanische Beinachse) wie oben beschrieben und äußert sich in einem geraden Bein, das so genannte Genu rectum (siehe Abb. 4, a). Als Beinachsenfehler werden Abweichungen von der physiologischen Norm bezeichnet. Diese können mit dem malalignement-Test analysiert werden (Paley und Pfeil 2000). Ein malalignement ist das Resultat einer mechanischen Achsdeviation (MAD). Bei einer Achsdeviation der mechanischen Tragachse vom Hüftkopfzentrum zum Mittelpunkt des oberen Sprunggelenks nach lateral vom 4-mm-Punkt des Kniegelenkzentrums nach Paley und Pfeil liegt ein so genanntes X-Bein, das Genu valgum (siehe Abb. 4, b) vor (Paley und Pfeil 2000). Besteht eine MAD der Mikulicz-Linie nach medial des Kniegelenkzentrums spricht man von einem O-Bein, Genu varum (siehe Abb. 4, c). Eine signifikante MAD besteht bei einer Verschiebung um $>10 \mathrm{~mm}$ nach lateral (Valgusfehlstellung) und bei $>15 \mathrm{~mm}$ nach medial (Varusfehlstellung) vom Kniegelenkszentrum (Paley und Pfeil 2000).

Bei pathologischen Achsabweichungen erfolgt dementsprechend die Lastübertragung nicht mehr gleichmäßig auf das mediale und das laterale Kniegelenkkompartiment. Die mechanische Überbelastung des jeweiligen Kompartimentes korreliert bei unphysiologischer Lastverteilung mit der Knorpelabnutzung (Cicuttini et al. 2004). Sharma et al. konnten in einer Studie den Zusammenhang zwischen malalignement und der Progression einer Gonarthrose zeigen (Sharma et al. 2001). 
Beinachsenfehlstellungen gelten daher in der Literatur als präarthrotische Deformitäten (Weseloh und Swoboda 2005). Aber nicht jedes X- oder O-Bein führt letztendlich zu einer Gonarthrose.

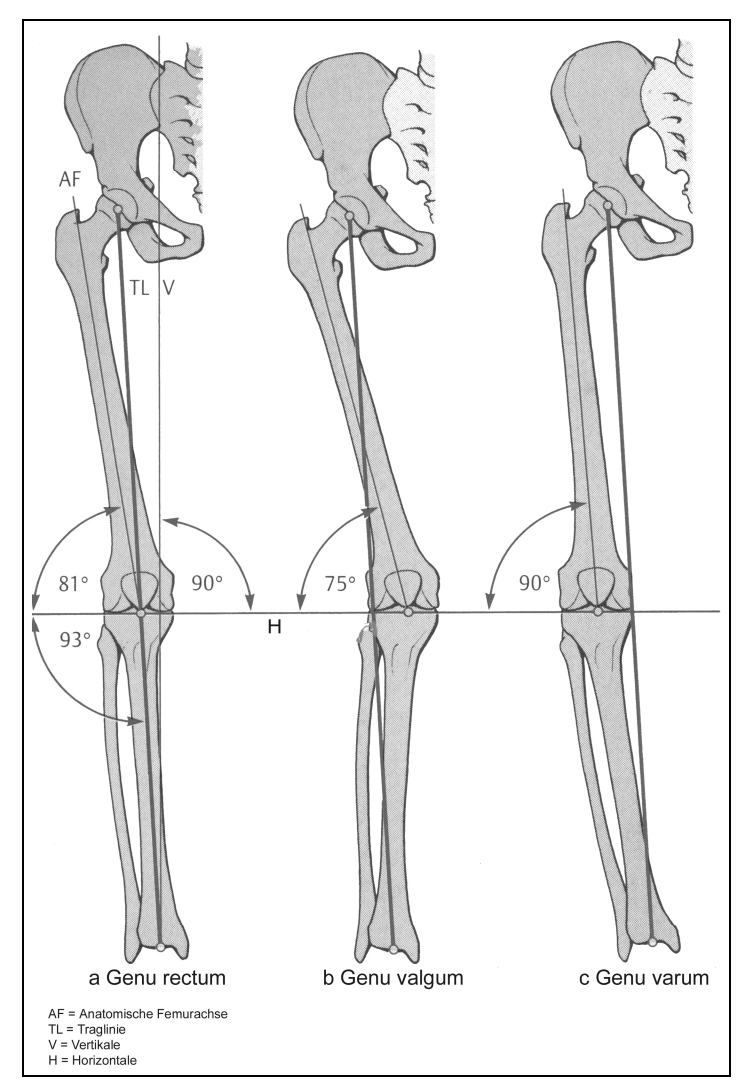

Abbildung 4: Beinachse des Menschen (modifiziert aus Niethard und Pfeil 1989, Seite 166, mit freundlicher Genehmigung des Hippokrates-Verlags)

\subsection{Gonarthrose - Die Arthrose im Kniegelenk}

Unter Arthrose (nach dem griechischen Wort „arthron“ für Gelenk) wird allgemein der zunehmende Knorpelverschleiß eines Gelenkes unter Beteiligung des Knochens verstanden, der altersabhängig chronisch progredient sowie bei stärkerer Gelenkveränderung zum Teil schmerzhaft verläuft. Das am häufigsten von degenerativen Veränderungen betroffene Gelenk ist das Kniegelenk (Debrunner 2005). Alle degenerativen Erkrankungen des Kniegelenks, sowohl femorotibial als auch femoropatellar, werden unter dem Terminus Gonarthrose zusammengefasst und gehen per definitionem mit einer progressiven Zerstörung des Gelenkknorpels unter Beteiligung der Gelenkstrukturen wie Knochen, synovialer und fibröser Gelenkkapsel sowie periartikulärer Muskulatur einher (DGOOC 2002). 
Die Gonarthrose und genauer differenzierte Untergruppen sind in der ICD-10Klassifikation der Weltgesundheitsorganisation unter dem Code M17 zu finden (DIMDI 2012). Zunächst kann die Gonarthrose isoliert das mediale bzw. laterale Kompartiment oder das Femoropatellargelenk betreffen, im fortgeschrittenen Stadium breitet sie sich meist auf alle drei Kompartimente aus (Pangonarthrose). Unterschieden werden die primäre (idiopathische) Gonarthrose, deren Ätiologie und Pathogenese unbekannt sind, sowie die sekundäre Gonarthrose (DGOOC 2002). Als Ursachen einer solchen sekundären Gonarthrose kommen mechanische, traumatische, entzündliche, metabolische oder auch neurologische Ursachen in Betracht (DGOOC 2002; Kwiatkowski 2004). Dadurch wird die Knorpelsubstanz geschädigt und die destruierende Kaskade einer Arthrose eingeleitet. Sie mündet in einer irreversiblen Knorpeldegeneration mit den röntgenologisch typischen Zeichen einer Gonarthrose. Zu diesen gehören die Gelenkspaltverschmälerung durch den Knorpeluntergang und die Deformierung des gelenkbildenden Knochens sowie die Knochenverdichtung im Gelenkflächenbereich (Sklerose). Ebenso kennzeichnend für das Bild einer Gonarthrose sind osteophytäre Anlagerungen (Exophyten) und Knochenhohlräume, so genannte Knochenzysten. Eine entsprechende radiologische Einteilung der Arthrose in vier Stadien (siehe Tab. 1) wurde von Kellgren und Lawrence vorgenommen (Kellgren und Lawrence 1957).

\begin{tabular}{|l|l|}
\hline Stadium & Röntgenkriterien \\
\hline $\mathbf{1}$ & $\begin{array}{l}\text { Es besteht eine geringe subchondrale Sklerosierung ohne Osteophytenbildung. Der } \\
\text { Gelenkspalt ist nicht verschmälert. }\end{array}$ \\
\hline $\mathbf{2}$ & $\begin{array}{l}\text { Hier liegt eine geringe Gelenkspaltverschmälerung vor. Zudem sind beginnende } \\
\text { osteophytäre Anbauten sowie eine angedeutete Konturunregelmäßigkeit der } \\
\text { Gelenkfläche zu finden. }\end{array}$ \\
\hline $\mathbf{3}$ & $\begin{array}{l}\text { Es zeigt sich eine ausgeprägte Osteophytenbildung mit Gelenkspaltverschmälerung. Die } \\
\text { Gelenkfläche weist eine deutliche Unregelmäßigkeit auf. }\end{array}$ \\
\hline $\mathbf{4}$ & $\begin{array}{l}\text { Der Gelenkspalt ist ausgeprägt verschmälert oder aufgehoben. Das Gelenk ist } \\
\text { vollständig destruiert. Es besteht eine Deformierung der Gelenkanteile. }\end{array}$ \\
\hline
\end{tabular}

Tabelle 1: $\quad$ Radiologische Einteilung der Arthrose nach Kellgren und Lawrence

Im Verlauf der arthrotischen Veränderungen treten zusätzlich phasenweise reaktive Entzündungen der Synovialis auf. 
Je nach Ausprägung der Gonarthrose wird die Varus- von der Valgusgonarthrose unterschieden. Von einer Varusgonarthrose spricht man, wenn der Substanzverlust überwiegend medial lokalisiert ist, woraus eine Varusstellung im Kniegelenk resultiert. Ergo ist die Valgusgonarthrose über einen lateralen Knorpeluntergang definiert. Als häufigste Form der Kniegelenksarthrose ist die Varusgonarthrose der älteren Frau zu beobachten (Debrunner 2005). Zur Behandlung der fortgeschrittenen Gonarthrose ist - bei entsprechender Indikation (Bewegungseinschränkung, Instabilität sowie Ruhe- und Belastungsschmerz) - die Implantation einer Kniegelenkstotalendoprothese seit drei Jahrzehnten eine etablierte Behandlungsmethode (Peterson et al. 1979).

\subsection{Hilfslinien zur Positionierung der Femurkomponente (knöcherne Landmarken)}

Um ein malalignement der Patella und eine Rotationsabweichung der Femurkomponente zu vermeiden, werden vorwiegend 3 knöcherne Landmarken (Hilfslinien) zur Rotationspositionierung der femoralen Implantatkomponente genutzt (Fehring 2000). Dabei werden folgende Hilfslinien unterschieden: 1.) die WhitesideLinie, 2.) die transepikondyläre Achse (TEA), 3.) die posteriore Kondylentangente bzw. Kondylenachse (PKA) (siehe Abb. 5).

Die Whiteside-Linie wurde von Leo A. Whiteside definiert als Linie, die senkrecht zur a.p.-Achse des distalen Femurs verläuft, wobei die a.p.-Achse die Verbindungslinie zwischen dem Zentrum der interkondylären Notch und dem tiefsten Punkt der Patellagrube darstellt (Whiteside und Arima 1995). Diese Linie soll der transepikondylären Achse entsprechen, jedoch zur Bestimmung besser sein (Whiteside und Arima 1995).

Die transepikondyläre Achse wurde 2000 von Kohn und Rupp nach Tastkontrolle und Augenmaß als Linie zwischen dem Zentrum des lateralen Epikondylus und dem sulkusartigen Zentrum des medialen Epikondylus beschrieben. Häufig ist sie weder eindeutig noch präzise festzulegen (Kohn und Rupp 2000). Anatomisch entsprechen die Endpunkte dieser Strecke den Ursprüngen des medialen und lateralen Seitenbandes (Berger et al. 1993). 
Hinsichtlich der Rotation der Femurkomponente wird diese Linie im Gegensatz zu den anderen Referenzachsen als zuverlässiger angesehen (Luo 2004). Dies impliziert eine annähernd normale Patellaführung und somit eine Minimierung der patellofemoralen Scherkräfte sowie eine Reduktion des tibiofemoralen Abriebs (Luo 2004).

In der Kniegelenksendoprothetik findet darüber hinaus die posteriore Kondylenachse häufig Verwendung als Referenzachse (Luo 2004). Diese Linie entspricht der Tangente der posterioren Femurkondylenoberfläche. Verschiedene Studien zeigen jedoch, dass es sich hierbei im Vergleich zur transepikondylären und zur a.p.-Achse, im klinischen Alltag auch als Whiteside-Linie bezeichnet, um eine weniger vertrauenswürdige Achse zur Einstellung der Femurrotation handelt (Poilvache et al. 1996; Miller et al. 2001).

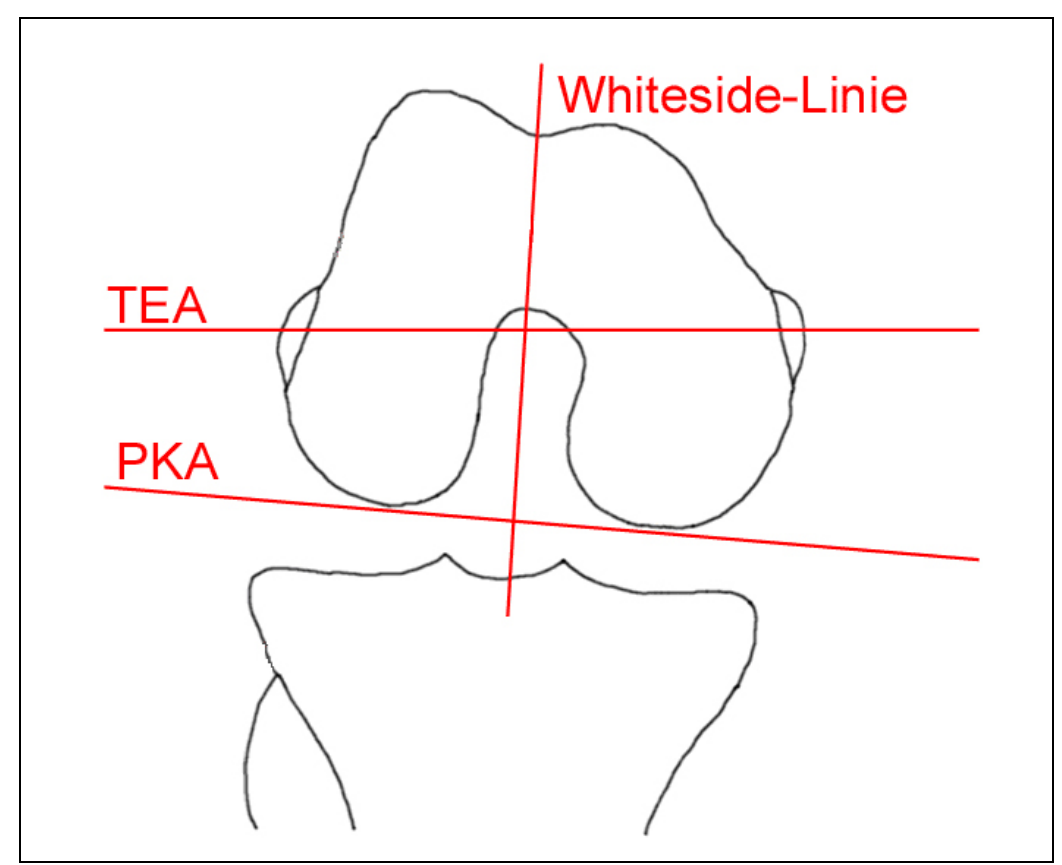

\section{Abbildung 5: Knöcherne Landmarken}

Das Tibiaplateau fällt zwar beim gesunden, „normalen“ Kniegelenk um $3^{\circ}$ nach medial ab, wird jedoch klassischerweise im Winkel von $90^{\circ}$ zur Tragachse der Tibia reseziert. Daher muss in Flexionsstellung des Kniegelenks an der medialen Femurkondyle verstärkt reseziert werden. Dies hat eine Außenrotation von $3^{\circ}$ in der Resektionsebene zur Folge. 
Nach Lüring et al. stellt die Methode der transepikondylären Achsenbestimmung und die damit einhergehende parallele Ausrichtung der Femurkomponente - eine signifikante Verbesserung des patellar trackings dar (Lüring et al. 2007). Allerdings ist keine der genannten Hilfslinien zur einwandfreien Rotationsausrichtung unproblematisch, da beträchtliche Messungenauigkeiten aufgrund anatomischer Verhältnisse bestehen (Kohn und Rupp 2000; Siston et al. 2005). Auch der Einsatz von Navigationsgeräten in der Kniegelenksendoprothetik konnte bisher keine verlässliche, reproduzierbare femorale Rotation bewirken (Siston et al. 2005).

Eine hohe Genauigkeit bei der Implantation ist aber unverzichtbare Voraussetzung für die Langlebigkeit von Kniegelenkstotalendoprothesen (Perlick et al. 2005). In verschiedenen Studien wird beschrieben, dass bereits geringe Abweichungen im alignement von mehr als $5^{\circ}$ Fehlrotation der Femurkomponente zu den knöchernen Landmarken mit einer erhöhten Versagensrate der Prothese verbunden sind und eine entsprechende Revisionsoperation erforderlich machen (Fehring und Valadie 1994; Hofmann et al. 2003; Restrepo et al. 2008).

\subsection{Einführung in die Thematik}

Innerhalb der letzten Jahre ist eine stetige Zunahme der diagnostizierten degenerativen Gelenkerkrankungen zu beobachten. Die jährlich veröffentlichten Statistiken der Bundesgeschäftsstelle Qualitätssicherung GmbH (BQS) spiegeln diese Tendenz anhand der Fallzahlen zur Erstimplantation von Totalendoprothesen wider. Mit mehr als 120.000 Primärimplantationen im Jahr 2006 stellt die Kniegelenksendoprothetik eine der häufigsten orthopädischen Operationen in Deutschland dar und gewinnt aufgrund der demographischen Entwicklung zunehmend an Bedeutung (BQS 2007). Der Lebensstil und die erhöhte Lebenserwartung, nicht nur in Deutschland, sondern in den westlichen Industrienationen insgesamt, gehen mit einer ansteigenden Zahl von Patienten mit Verschleißerscheinungen am Kniegelenk und einem Zuwachs der Implantationszahlen des künstlichen Kniegelenksersatzes einher (BQS 2007). Zudem besteht ein erhöhtes Risiko infolge früherer Traumata im Kniegelenksbereich, beruflicher Exposition und sportlicher Belastung auf Hochleistungsniveau. 
Die fortgeschrittene, schmerzhafte Gonarthrose ist der häufigste Grund für die Implantation einer Kniegelenkstotalendoprothese und gilt derzeit als "Goldstandard“ bei der Behandlung (Kowalski und Gorecki 2004). Analog den Leitlinien der Deutschen Gesellschaft für Unfallchirurgie ist bei Vorliegen einer signifikanten Einschränkung der Gehstrecke oder eines Dauerschmerzes die Indikation zur definitiven operativen Therapie gegeben (Stürmer 2001). Auch der sozialmedizinische Aspekt darf bei einer Prävalenz mit bis zu $90 \%$ bei den über 60 Jährigen nicht vernachlässigt werden (Sun et al. 1997; DGOOC 2002). Die Ziele der Therapie resultieren aus dem Beschwerdebild der degenerativen Kniegelenkserkrankung. Die Symptomatik mit schmerzhafter, eingeschränkter Beweglichkeit und Belastbarkeit führt letztlich zu einer erheblich verminderten Lebensqualität. Durch den Einsatz künstlicher Kniegelenke wird sowohl eine wesentliche Verbesserung der Mobilität als auch die angestrebte Schmerzfreiheit erreicht, was wiederum mit einem signifikanten Anstieg der Lebensqualität einhergeht (Heck et al. 1998; Jerosch und Floren 2000; König et al. 2000; Stevens et al. 2003). Dies verdeutlicht die Notwendigkeit einer kontinuierlichen Weiterentwicklung des Prothesendesigns und der Operationsmethodik, um annähernd anatomisch achsgerechte bzw. mechanische Verhältnisse zu schaffen. Bereits heutzutage werden in Langzeitstudien mit einem minimum follow-up von zehn Jahren hervorragende Prothesenstandzeiten erreicht (Pavone et al. 2001; Rodriguez et al. 2001; Robertsson et al. 2006).

Als Hauptursache für ein vorzeitiges Versagen der Prothese mit anschließender Revision gilt neben Infektion und aseptischer Lockerung die Fehlausrichtung des Implantats und die Bandinstabilität (Peterson et al. 1979; Townley 1985; Callaghan et al. 2004; Vessely et al. 2006; Claus und Scharf 2007; Graichen et al. 2007). Nach Sharkey et al. und Callaghan et al. korreliert insbesondere die Malrotation (Fehlrotation) der femoralen Implantatkomponente mit der Anzahl der Revisionen in der Kniegelenksendoprothetik (Sharkey et al. 2002; Callaghan et al. 2004). Für gute klinische Langzeitergebnisse ist folglich eine korrekte Positionierung der Prothesenkomponente(n) erforderlich. Die mechanisch korrekte Ausrichtung der Prothesenkomponenten hinsichtlich der Tragachse des Beines, das so genannte alignement, stellt weiterhin eine Herausforderung für den Operateur dar, obwohl sich die Operationstechniken stetig weiterentwickelt und verbessert haben. 
Eine durch Fehlrotation verstärkte Scherbelastung am Polyethylen-Inlay bedingt einen vermehrten Abrieb des Polyethylens (PE) (Kohn und Rupp 2000) und beeinflusst dadurch die Prothesenstandzeit. In diesem Zusammenhang kann ein asymmetrischer Beugespalt $\mathrm{zu}$ einer erhöhten einseitigen Bandspannung mit vermehrtem PE-Verschleiß oder zu einer unilateralen Instabilität bei Flexion führen (Kohn und Rupp 2000; Insall et al. 2002; Graichen et al. 2007; Romero et al. 2007). Ferner kann es infolge der Komponentenrotation zu einer mangelhaften Patellaführung, dem patellar maltracking, kommen, was erhebliche Schmerzen am Femoropatellargelenk zur Folge haben kann (Berger et al. 1998; Stöckl et al. 2004). Die exakte Rekonstruktion des Patellalaufs ist insofern von Bedeutung, da die Positionierung der Patella zugleich einen wesentlichen Anteil am Behandlungsergebnis hat (Büttner-Janz und Ekkernkamp 2002). Die Verwendung von intraoperativen Navigationssystemen hat einen besonderen Stellenwert in Bezug auf die Passgenauigkeit und Achsausrichtung in der Kniegelenksendoprothetik eingenommen (Kohn und Rupp 2000). Nach Bertsch et al. werden sie im klinischen Alltag mit zunehmender Häufigkeit eingesetzt (Bertsch et al. 2007). In mehreren Studien konnte ein präziseres alignement der Komponenten dank der Anwendung moderner Computernavigation realisiert werden (Oberst et al. 2003; Sparmann et al. 2003; Bäthis et al. 2004; Chauhan et al. 2004; Walde et al. 2005; Bäthis et al. 2006; Bejek et al. 2007)

\subsection{Die Bedeutung des Weichteilmanagements in der Kniegelenksendoprothetik}

Die Kollateralbänder spielen durch den Einsatz von Oberflächenersatzprothesen bei Primärimplantationen eine entscheidende Rolle, da sie aufgrund der teilweise kompletten Kreuzbandresektion maßgeblich an der Stabilität der Kniegelenkstotalendoprothese beteiligt sind und folglich einer hohen Beanspruchung unterliegen (Graichen et al. 2007). Ein intakter Bandapparat ist somit Voraussetzung für die Implantation einer bikondylären Prothese und beeinflusst zusätzlich die spätere Gelenkfunktion (Briard et al. 2007). Liegt eine zu straffe oder zu laxe Bandführung vor, wird dadurch entweder die Beweglichkeit eingeschränkt, ein vermehrter Polyethylenabrieb bis hin zur aseptischen Lockerung begünstigt oder eine unilaterale Instabilität hervorgerufen. 
Bei zu straffen Kollateralbändern besteht die Möglichkeit einer Korrektur durch Entlastung (release) der betroffenen Seite. Mit Hilfe der Einstellung der Weichteilspannung (ligament balancing) bzw. Weichteilbalancierung kann ein funktionstüchtiger, „balancierter“ Bandapparat wiederhergestellt werden, der für einen künstlichen Oberflächenersatz des Kniegelenks erforderlich ist (Kohn und Rupp 1999). Jedoch fehlen gesicherte Daten über Messwerte der physiologischen Bandspannung. In verschiedenen Kadaverstudien werden ca. 100 Newtonmeter in Extension und 75 Newtonmeter in Flexion angenommen (Jerosch et al. 2007). Der Begriff Weichteilmanagement impliziert das ligament balancing und die bandspannungsbasierte Auswahl der Implantate. Das Weichteilmanagement stellt ein sehr komplexes Gebiet der Kniegelenksendoprothetik dar und ist von entscheidender Bedeutung für die erfolgreiche Implantation respektive den Behandlungserfolg (Kohn und Rupp 2000). Trägt der Kollateralbandapparat nicht ausreichend zur Stabilität bei, muss der Einsatz von teilgekoppelten oder gekoppelten Prothesensystemen in Erwägung gezogen werden.

\subsection{Computergestützte Navigation in der modernen Kniegelenksendoprothetik}

Durch den Einsatz von Navigation in der Kniegelenksendoprothetik stehen dem Operateur hochpräzise intraoperative Informationen über die individuellen Achsverhältnisse, die Bandstabilität und die Kinematik des Patientengelenks zur Verfügung. Unter Fixierung von Lokatoren am Knochen kann der Computer jederzeit alle gespeicherten Landmarken im Raum orten und alle wichtigen Größen ermitteln. Durch Anwendung einer speziellen Software entsteht auf dem Monitor des genutzten Computer-Terminals nach Registrierung der konventionellen knöchernen Landmarken sowie weiterer definierter Messpunkte ein dreidimensionales Modell der Patientenanatomie, aus dem Lage und Winkel der Gelenkkongruenten zueinander ablesbar sind. Bei der Planung am Navigationssystem wird anhand der dynamisch erfassten Informationen wie Gelenkbewegung, Gelenkrotation und Winkel die Wahl der Implantatgrößen frühzeitig miteinbezogen. Zusätzlich kann die Weichteilbalancierung zur Implantatpositionierung berücksichtigt werden. 
Dadurch können zur Festlegung der Knochenresektion hinsichtlich der Ausrichtung der Implantatkomponenten sowohl die knöchernen als auch die ligamentären Strukturen benutzt werden (Oberst et al. 2006; Claus und Scharf 2007). Alle geplanten Schnitte können während der Durchführung in Realzeit auf ihre Genauigkeit überprüft werden und bei Bedarf bis zur optimalen Position der Sägelehren nachgebessert werden (Bäthis et al. 2006). Folglich kann die computergestützte Navigation den Operateur im Gegensatz zur konventionellen Operations-Technik zu jedem Zeitpunkt des Eingriffs über eine Fehlresektion unterrichten und ermöglicht somit eine intraoperative Korrektur, wodurch Schnittfehler minimiert werden können (Bäthis et al. 2005). Mit dieser Technik kann der OP-Verlauf optimal abgebildet und die jeweilige Situation bestmöglich berücksichtigt werden (Stulberg et al. 2006). Ebenso werden bei der Navigation bestimmte Schritte durch einzeitige Planung und Ausführung optimiert und peri-/ postoperative Komplikationen reduziert. Eine vergleichende randomisierte kontrollierte Studie von Kalairajah et al. bestätigt, dass die computergestützte Implantation die Inzidenz von (Fett-) Embolien in der Kniegelenksendoprothetik durch den Verzicht auf intramedulläre Ausrichtsysteme verkleinert (Kalairajah et al. 2006). Mit einer Häufigkeit von über 50\% sind Instabilitäten und eine Fehlausrichtung der Prothesenkomponenten die wesentlichen Ursachen der Revisionsfälle innerhalb der ersten zwei Jahre nach Primärimplantation (Sharkey et al. 2002). Zur Vermeidung von Prothesenfehlpositionierungen hat sich der computerassistierte Kniegelenkersatz bewährt (Nabeyama et al. 2004; Sikorski 2004; Pitto et al. 2006; Stiehl 2007). Eine korrekte Implantatpositionierung mit der Rekonstruktion einer postoperativen mechanischen Beinachse in einem Korridor von $3^{\circ}$ Varus und $3^{\circ}$ Valgus ist im Unterschied zur konventionellen OP-Technik hoch signifikant (Bäthis et al. 2006). Durch Prozessoptimierung wird die Operationsdauer bei der navigierten Implantation von Kniegelenkstotalendoprothesen im Vergleich zum konventionellen Verfahren nicht verlängert (Walde et al. 2005). Trotzdem macht die Navigation einen sehr geringen Anteil an den jährlich durchgeführten Primärimplantationen aus. Unter anderem liegen die Gründe hierfür sicherlich in der Höhe der Investitionskosten und dem enormen Kostendruck, dem sich die Krankenhäuser ausgesetzt sehen. 


\subsection{Hypothesen und Ziel der Studie}

Seit 01.01.2003 wird im Nordwest-Krankenhaus Sanderbusch GmbH die computergestützte Navigation in der Kniegelenksendoprothetik eingesetzt. Seit 01.05.2006 wird die Navigation unter Berücksichtigung der Bandspannung des Kniegelenks verwendet. Dabei erfolgt ein verändertes operatives Vorgehen mit einer bandspannungsbasierten femoralen Rotationsausrichtung in der so genannten tibia first-Technik unter konsekutiver Verwendung eines Bandspanners. Zusätzlich werden die 3 konventionellen knöchernen Landmarken zur femoralen Rotationsausrichtung während der Operation digital erfasst und mit der bandspannungsbasierten navigierten Rotationsausrichtung verglichen.

\section{Hypothese 1:}

Bei der Primärimplantation wird mit der bandspannungsbasierten femoralen Rotationsausrichtung unter Navigationskontrolle eine Fehlrotation der Femurkomponente mit einer negativen Beeinflussung der Funktion der Kniegelenkstotalendoprothese verhindert und eine funktionell korrekte Positionierung erreicht.

\section{Hypothese 2:}

Mit Hilfe der Studiennavigationstechnik können die bisherigen knöchernen Landmarken des Femurs mit der bandspannungsbasierten Rotationsausrichtung simultan verglichen werden.

\section{Ziel der Studie:}

Inwiefern sind die knöchernen Landmarken und die bandspannungsbasierte navigierte OP-Technik zur Rotationsausrichtung der femoralen Implantatkomponente hinsichtlich ihres funktionellen Ergebnisses bei synchroner Überprüfung verlässlich. Direkte Empfehlungen für den klinischen Alltag sollen durch die Studienergebnisse erfolgen. 


\section{Material und Methodik}

\subsection{Das EFK ${ }^{\circledR}$-Kniesystem}

Das in der vorliegenden Studie verwendete Totalendoprothesensytem EFK ${ }^{\circledR}$ der Smith \& Nephew GmbH ist ein ungekoppelter bikondylärer Oberflächenersatz für die primäre Implantation (siehe Abb. 6).

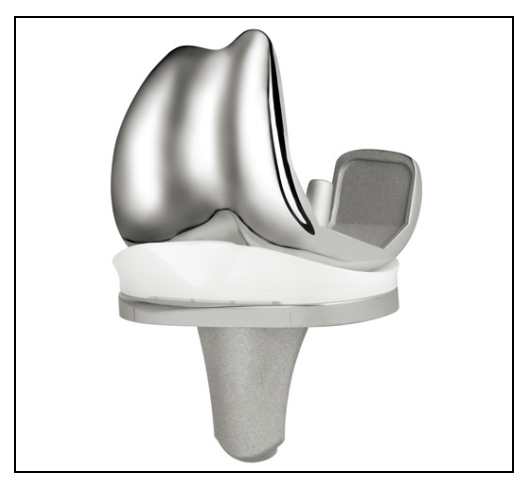

Abbildung 6: Prothese EFK ${ }^{\circledR}$ (aus Operationsanleitung EFK ${ }^{\circledR}$-Kniesystem 2008, Seite 5, mit freundlicher Genehmigung der Smith \& Nephew GmbH)

Sowohl die femorale als auch die tibiale Komponente werden aus einer CobaltChrom-Molybdän (CoCrMo)-Gusslegierung nach ISO 5832-4 hergestellt. Die Femurkomponente ist asymmetrisch mit einer tiefen, langgezogenen und schräg verlaufenden Patellagrube sowie einem Patellaschild in $3^{\circ}$-Neigung nach lateral geformt. Dadurch soll eine verbesserte Patellaführung mit Reduktion der femoropatellaren Anpresskräfte zur Vermeidung eines patellar maltracking erzielt werden. An der Rückfläche befinden sich zwei abgewinkelte Zapfen zur Rotationssicherung. Die Krümmungsradien der femoralen Komponente sind physiologisch gestaltet, so dass auch bei Beugegraden über $90^{\circ}$ eine optimale Kongruenz vorliegen soll. Zur Minimierung der Gefahr eines notching (Einkerben der ventralen Kortikalis beim Sägeschnitt) steigt das Patellaschild an der Rückfläche um 6o nach ventral-proximal an. Die tibiale Komponente ist zur vollständigen kortikalen Abstützung auf der Resektionsebene ebenfalls asymmetrisch gestaltet. Die tibiale Basis soll somit bei korrekter Resektion eine optimale Kongruenz zur knöchernen Auflage erzielen. Zur Stabilisierung wird die unzementierte Komponente mit zwei Sicherungsschrauben in der Spongiosa fixiert. 
Das PE-Inlay ist ein ultrahochmolekulares Polyethylen nach ISO 5834-2, das sich laut Hersteller besonders durch seine Lastdämpfungsfähigkeit bei geringer Reibung und äußerst geringem Verschleiß auszeichnet. Kondylenseitig besitzt das modulare PE-Inlay ein symmetrisches Design. Darüber hinaus wurde es tribologisch optimiert, um eine homogene Druckverteilung über eine großflächige, ovale Kontaktzone zu erreichen. Als Retropatellarersatz kann optional eine Komponente aus VollPolyethylen mit symmetrischer, konvexer Oberfläche verwendet werden.

\subsection{Die Operationstechnik mit dem Navigationssystem PiGalileo TKR Light ${ }^{\circledR}$, Version 1.3}

Die präoperative Planung erfolgt anhand einer Ganzbeinröntgenaufnahme der betroffenen Extremität im Zweibeinstand anterior-posterior (Bernau 1995). Es wird die Belastungsachse "C“ eingezeichnet, die beim geraden Bein vom Mittelpunkt des Femurkopfes durch das Zentrum des Kniegelenks bzw. $4 \mathrm{~mm}( \pm 2 \mathrm{~mm})$ medial des Kniegelenkszentrums nach Paley und Pfeil zum Mittelpunkt des oberen Sprunggelenkes führt (Paley und Pfeil 2000). Sie entspricht der femoralen Traglinie „D“ und der tibialen Traglinie „B“, sprich der mechanischen Achse. Außerdem erfolgt die Festlegung der anatomischen Femurachse „A“. Zwischen anatomischer Femurachse und femoraler Traglinie entsteht ein Winkel „a“, der physiologisch $6^{\circ}-7^{\circ}$ beträgt (siehe Abb. 7).

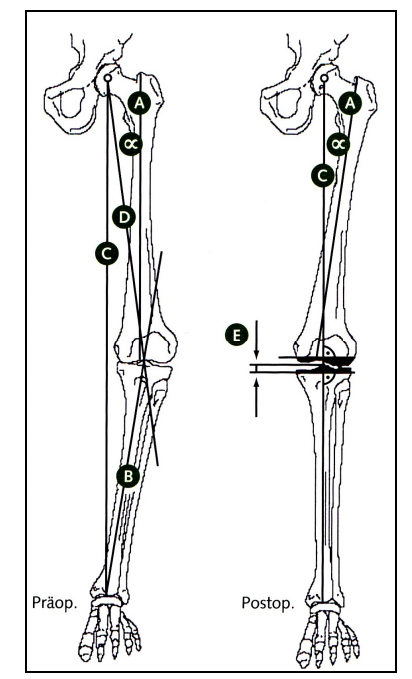

Abbildung 7: Schematische Übersicht der Achs- und Winkelverhältnisse (aus Operationsanleitung $E F K^{\circledR}-$ Kniesystem 2008, Seite 9, mit freundlicher Genehmigung der Smith \& Nephew GmbH) 
Die Implantation der EFK ${ }^{\circledR}$-Kniegelenkstotalendoprothese (Smith \& Nephew GmbH) wird im Rahmen dieser Studie in navigierter Technik mit dem System PiGalileo TKR Light $^{\circledR}$, Version 1.3 der Firma Smith \& Nephew GmbH durchgeführt und dokumentiert. Anhand von Referenzlokatoren an Femur und Tibia werden individuelle Vertrauenspunkte durch eine vorher positionierte Kamera des Navigationssystems digital erfasst und auf dem Monitor angezeigt. Die Messungen erfolgen nach einem genau definierten Schema. Durch Aufzeichnung der Daten werden dem Operateur die korrekt ausgerichteten Resektionslinien präsentiert. Zur Festlegung des proximalen Punktes der mechanischen Achse - dem Mittelpunkt des Femurkopfes - wird zuerst eine Kinematikanalyse mit Beinpositionen in verschiedenen Flexions- und Ab-/Adduktionsstellungen durchgeführt und mit Hilfe von Lokatoren registriert. Während dieser Messung sollte sich das Becken nicht bewegen, damit die Punktbestimmung im Raum eindeutig erfolgt. Die weiteren Landmarken am Femur werden mittels eines mobilen Lokators bestimmt. Danach werden die Messpunkte an der Tibia erfasst. Unter Lokatorenkontrolle werden die Sägeblöcke präzise für die Resektion justiert. Mit dem Navigationssystem besteht die Möglichkeit neben der entsprechend den knöchernen Landmarken referenzierten OP-Technik femur first oder tibia first zudem die Option, tibia first mit gap adjustment zu operieren. In diesem Fall wird im Gegensatz zur tibia first-Variante bei der Wahl des PE-Inlays, der femoralen Implantatgröße und der Rotation der femoralen Implantatkomponente die Bandspannung mitberücksichtigt. Zur Messung wird ein entsprechender Bandspanner benutzt. Dieses Verfahren ist Inhalt der durchgeführten Studie. Alle Operationsergebnisse werden intraoperativ erfasst. Ein Funktionsdiagramm zeigt nach Registrierung der Referenzpunkte und Entfernung von eventuellen Osteophyten den Bewegungsumfang (ROM) hinsichtlich Extension und Flexion mit jeweiliger Varus- bzw. Valgusstellung vor der Resektion sowie nach Implantation der Prothese. Diese Ergebnisse werden vom Navigationscomputer protokolliert und in die Studie einbezogen. Nach dem Starten der Navigationssoftware an dem mobilen Computer-Terminal, das über eine Kamera verfügt (siehe Abb. 8), können durch den Operateur alle Menübefehle mit einem Fußschalter eingegeben werden. Die Kamera, immer relativ zum operierenden Bein ausgerichtet, wird medial zum Operationsfeld positioniert. Die optimale Distanz liegt bei ca. $180-200 \mathrm{~cm}$ und die ideale Höhe bei $180 \mathrm{~cm}$ zum Operationsgebiet. 
Das Ausrichten der Kamera erfolgt durch einen Laserpointer und/oder anhand der Lokatoren.

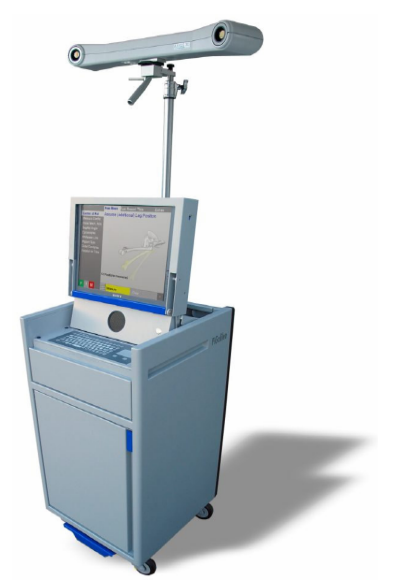

Abbildung 8: Navigationssystem PiGalileo TKR Light $^{\circledR}$, Version 1.3 (aus Operationsanleitung PiGalileo TKR Light $^{\circledR}$ 2006, Seite 2, mit freundlicher Genehmigung der Smith \& Nephew GmbH)

Anschließend wird die OP-Reihenfolge wie femur first, tibia first oder, wie in der Studie angewendet, tibia first unter Berücksichtigung der Bandspannung (gap adjustment) festgelegt. Dadurch wird die Ausführung zusätzlicher Funktionen und Messungen im OP-Verlauf ermöglicht. Eine korrekte Ausrichtung der Kamera sowie die Erfassung des femoralen $(F)$, tibialen $(T)$ sowie mobilen $(M)$ Referenzlokators wird dem Operateur durch ein entsprechendes akustisches Signal mitgeteilt. Die Position des jeweiligen Lokators wird durch einen farblich zugeordneten Ring dargestellt (siehe Abb. 9). Nach Abschluss dieser Aktion findet eine Initialisierung des Systems statt.

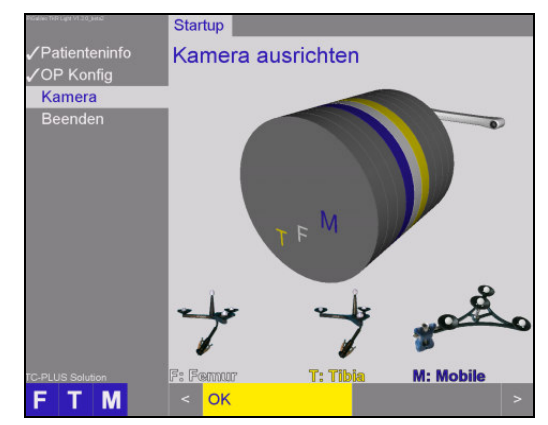

Abbildung 9: Ausrichten der Lokatoren (aus Operationsanleitung PiGalileo TKR Light ${ }^{\circledR}$ 2006, Seite 7, mit freundlicher Genehmigung der Smith \& Nephew GmbH) 
Nach Lagerung des Patienten in Rückenlage und Anlegen bzw. Schließen einer pneumatischen Oberschenkelblutsperre erfolgt der Hautschnitt als midline incision. Die Haut, das Subkutanfettgewebe und die Faszie werden durchtrennt. Daran schließt sich nach Darstellung der Patella eine mediale parapatellare Arthrotomie zur möglichen Luxation der Patella nach lateral mit Inzision nach proximal in die Quadrizepssehne hinein sowie nach distal bis zur Tuberositas tibiae reichend an. Dabei wird der Vastus medialis des Musculus quadriceps femoris geschont. Nach Inspektion wird das Bein in maximaler Flexion aufgestellt und beide Menisci, das vordere Kreuzband und Teile des Hoffa-Fettkörpers werden reseziert. Arthrotische Exophyten werden im Sinne einer Cheilotomie abgetragen, gegebenenfalls erfolgt eine Synovektomie. Das distale Femurende sowie die Fossa intercondylaris werden dargestellt. Bei Freilegung des medialen Tibiaplateaus wird das Ligamentum meniscotibiale zur erleichterten tibialen Resektion tangential zum Knochen mit abgelöst. Dies entspricht einem erstgradigen medialen release. Im weiteren Verlauf werden die Referenzlokatoren am distalen Femur direkt im Zugang und an der medialen Tibiakante mittels einer transkutanen Lokatorplatte (in ca. $30^{\circ}$ Neigung Richtung Kamera) angebracht.

\subsubsection{Messungen am Femur zur Bestimmung der knöchernen}

\section{Landmarken}

Durch Betätigen des Fußschalters werden die jeweiligen Messungen ausgelöst und registriert. Die spezifischen Punktmessungen erfolgen anhand des mobilen Lokators. Eine Kinematikanalyse aus mindestens sechs Beinpositionen in verschiedenen Flexions- und Add- bzw. Abduktionsstellungen bestimmt das Rotationszentrum der Hüfte. Zu Beginn werden zwei Messungen in Extension durchgeführt, anschließend wird jeweils noch zwei bis drei weitere Male bei $45^{\circ}$ und $90^{\circ}$ gemessen, um den proximalen Punkt der mechanischen Achse des Femur zu ermitteln. Laut Hersteller beträgt die Genauigkeit der Bestimmung +/- 2º (Operationsanleitung PiGalileo TKR Light $^{\Theta}$ 2006). Für die distale Definition der mechanischen Femurachse ist der Durchstoßpunkt auf der Knorpelfläche im Zentrum des Kniegelenks mit dem mobilen Lokator aufzusuchen. Der Durchtrittspunkt befindet sich über der interkondylären Notch am dorsalsten Anteil der Trochlea (siehe Abb. 10 und 11). 


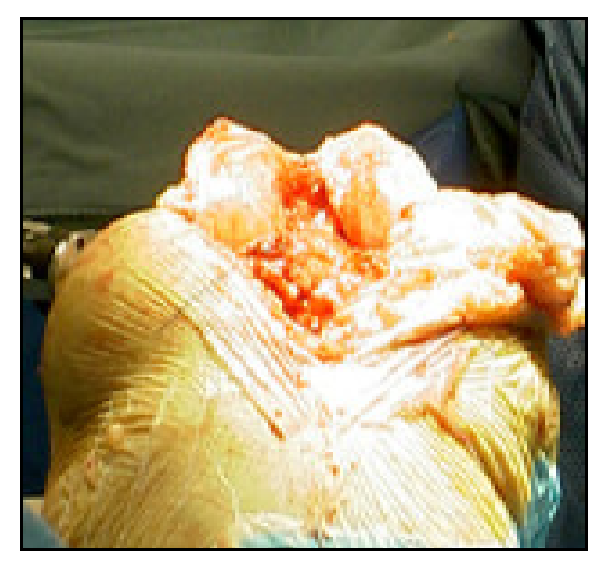

Abbildung 10: In-vivo-Aufnahme bei flektiertem Kniegelenk zur Bestimmung der mechanischen Femurachse (aus Operationsanleitung PiGalileo TKR Light ${ }^{\circledR}$ 2006, Seite 15, mit freundlicher Genehmigung der Smith \& Nephew GmbH)

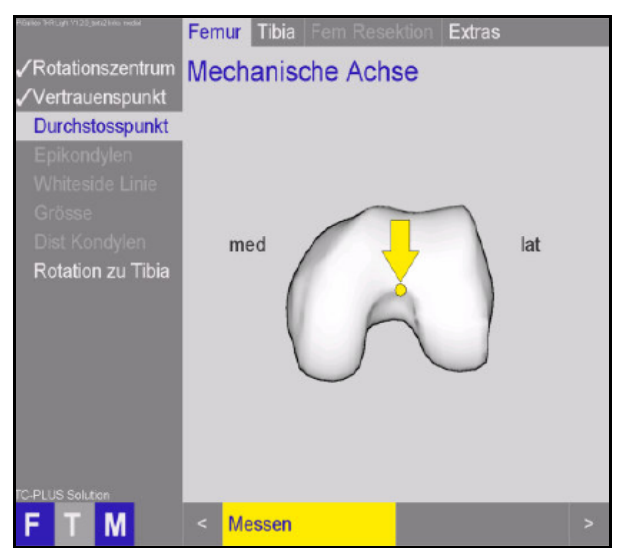

Abbildung 11: Monitorbild Durchstoßpunkt der mechanischen Achse am distalen Femur (aus Operationsanleitung PiGalileo TKR Light ${ }^{\circledR} 2006$, Seite 15 , mit freundlicher Genehmigung der Smith \& Nephew GmbH)

Im Anschluss hieran wird die transepikondyläre Achse (TEA) bestimmt, indem der mediale Epikondylus im Zentrum des Sulkus und die prominenteste Erhebung des lateralen Epikondylus mit dem mobilen Lokator aufgesucht und dann vom Navigationssystem erfasst werden. Die TEA stellt eine der oben bereits beschriebenen 3 knöchernen Landmarken am Femur zur Rotationsausrichtung der femoralen Implantatkomponente dar. Als nächstes erfolgt die Aufzeichnung der Whiteside-Linie durch Messungen von Tastpunkten auf einer Länge von 2,5 cm senkrecht zur TEA zwischen dem ventralsten Punkt (anteriorer Whiteside-Punkt) und dem tiefsten Punkt der Femurtrochlea (siehe Abb. 12 und 13). 


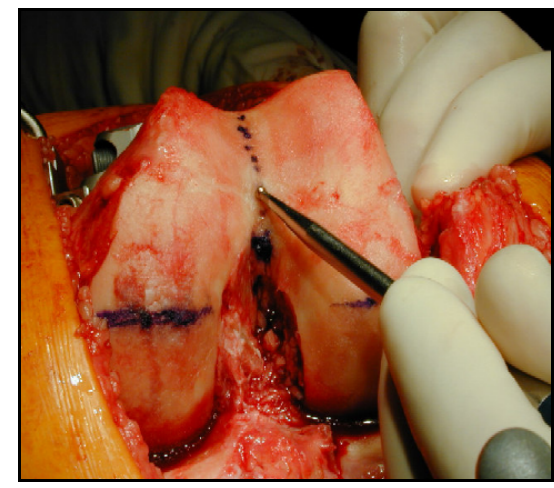

Abbildung 12: In-vivo-

Aufnahme bei Bestimmung der

Whiteside-Linie (aus

Operationsanleitung PiGalileo

TKR Light ${ }^{\circledR}$ 2006, Seite 16, mit

freundlicher Genehmigung der

Smith \& Nephew GmbH)

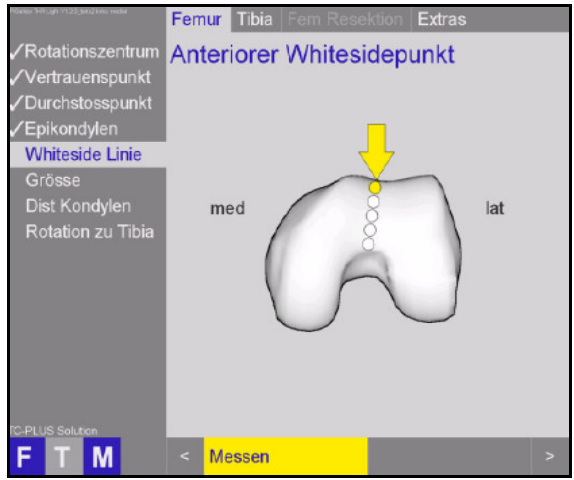

Abbildung 13: Monitorbild Tastpunkte der Whiteside-Linie (aus Operationsanleitung PiGalileo TKR Light ${ }^{\circledR}$ 2006, Seite 16, mit freundlicher Genehmigung der Smith \& Nephew GmbH)

Bevor die letzte knöcherne Landmarke, die posteriore Kondylenachse, gemessen wird, muss ein anteriorer Referenzpunkt für die anteriore Position der Femurkomponente ermittelt werden, der als zusätzliche Landmarke für die Wahl der späteren Implantatgröße dient. Dadurch sollen ventrale Kerbungen am Femur (notching) vermieden werden. Nun werden die Messungen der medialen und lateralen posterioren Kondylen zur Definierung der PKA durchgeführt. Ebenso wird der distalste Punkt der jeweiligen Kondyle mit Hilfe eines Lokators bestimmt, der zur mechanischen Achse ausgerichtet ist. Damit sind die Messungen am Femur abgeschlossen.

\subsubsection{Navigationsbasierte Festlegung der tibialen Resektion und Messung des präoperativen Bewegungsumfanges}

Für die Definition des proximalen Punktes der tibialen mechanischen Achse wird der Lokator zwischen den Spitzen der Eminentia tibiae (dies entspricht dem Durchtrittspunkt der mechanischen Achse) aufgesetzt (siehe Abb. 14). Die tibiale mechanische Achse entspricht der linearen Verlängerung der mechanischen Femurachse. 


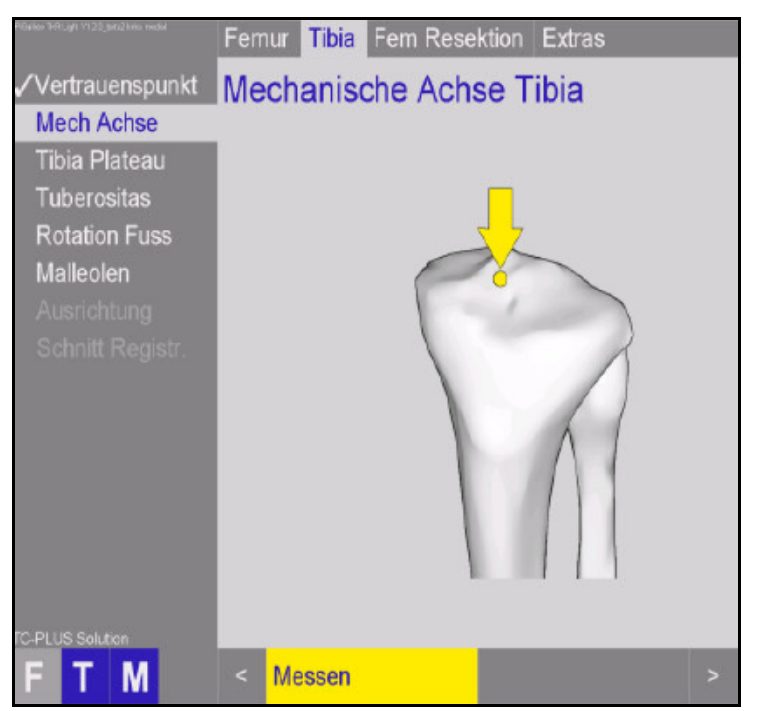

Abbildung 14: Monitorbild - proximale mechanische Achse der Tibia (aus Operationsanleitung PiGalileo TKR Light ${ }^{\circledR}$ 2006, Seite 24, mit freundlicher Genehmigung der Smith \& Nephew GmbH)

Im weiteren Operationsverlauf werden per mobilem Lokator der tiefste Punkt des Gelenkdefekts und die intakte kontralaterale Kondyle als Referenzwert für die Gelenkebene (joint level) zur Bestimmung der tibialen Resektionshöhe definiert. Anschließend erfolgt die Festlegung der tibialen Rotation in Richtung der Tuberositas tibiae, indem der Lokator in paralleler Ausrichtung zum Tibiaplateau auf den Punkt der mechanischen Achse über das mediale Drittel der Tuberositas gelegt wird. Anhand des mobilen Lokators auf der Sehne des Musculus tibialis anterior mit Ausrichtung über den zweiten metatarsalen Strahl nach anterior wird eine zusätzliche tibiale Rotationslandmarke aufgezeichnet. Der distale Punkt der tibialen mechanischen Achse wird durch Messung der prominentesten Stelle des medialen Malleolus und des distalsten Punkts der Fibulaspitze (lateraler Malleolus) eingelesen.

Nach abgeschlossener Registrierung der knöchernen Landmarken wird vor Beginn der Resektion ein Funktionsdiagramm erstellt.

Das Funktionsdiagramm zeigt den Bewegungsumfang (ROM) hinsichtlich Extension und Flexion mit jeweiliger Varus- bzw. Valgusstellung in einem Koordinatensystem. Die gespeicherten Daten dieses Funktionsdiagramms sind Bestandteil der Studie (siehe Abb. 15). 


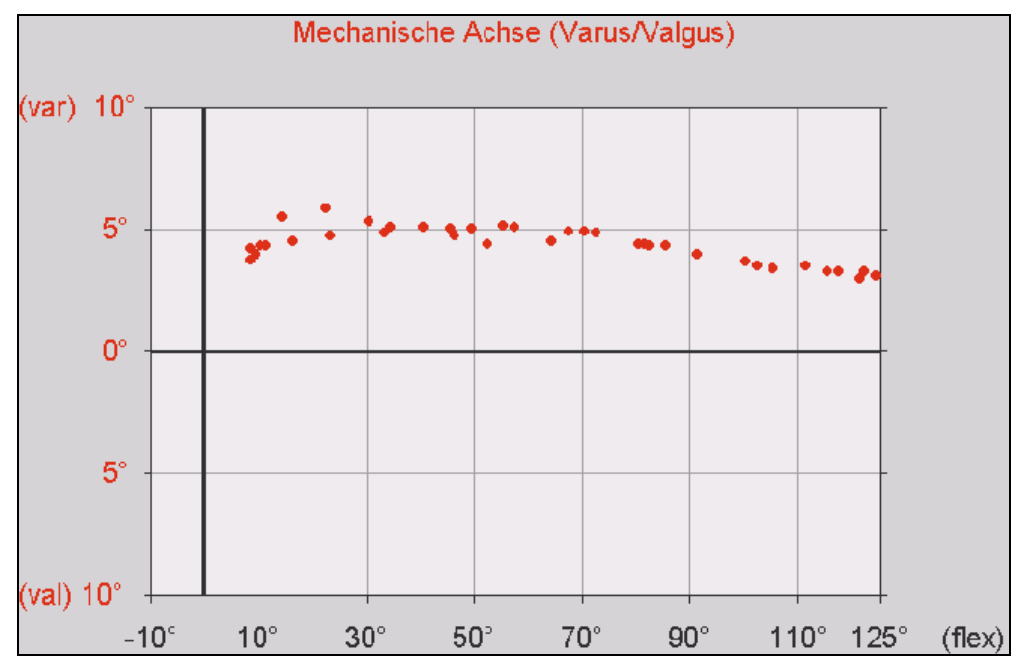

Abbildung 15: Präoperative ROM-Messung (Beispielabbildung eines Studienpatienten)

Unter Navigationskontrolle wird nun der tibiale Schnittblock an der proximalen Tibia fixiert. An diesem Schnittblock kann zusätzlich zu den üblichen Verstellmöglichkeiten eine navigationsbasierte Feinjustierung vorgenommen werden. Bei der Ausrichtung des Schnittblockes werden verschiedene Daten registriert - darunter die Rotation, der tibiale slope, die Resektionshöhe medial und lateral sowie die Varus- und Valgusachse der geplanten Resektion. Die tibiale Resektion wird auf der Datengrundlage dieser Messungen visualisiert und dementsprechend durchgeführt.

\subsubsection{Bestimmung der Rotationsausrichtung der femoralen Implantatkomponente mit bandspannungsbasierter Navigation}

Die Festlegung der Femurresektion beginnt mit dem in Streckstellung und unter Navigation eingebrachten distalen Sägeblock. Nach navigationsunterstützter Feinjustierung senkrecht zur mechanischen Femurachse in $2^{\circ}$-Flexion zur Vermeidung eines ventralen notchings wird der distale Femurschnitt ausgeführt. Durch einen speziellen Bandspanner wird die Parallelität der Resektionsflächen überprüft (siehe Abb. 16). Dieser wird nach vorheriger Vorspannung mit jeweils 90 Newton medial und lateral in Extension eingesetzt. In Abhängigkeit der Bandspannung und des Abstands zwischen Femur und Tibia wird mit dieser Methode die Größe des PE-Inlays und somit die Stabilität in Extension ermittelt. Die aufzuwendende Kraft des Bandspanners ist vor Studienbeginn mit 90 Newton definiert worden. 


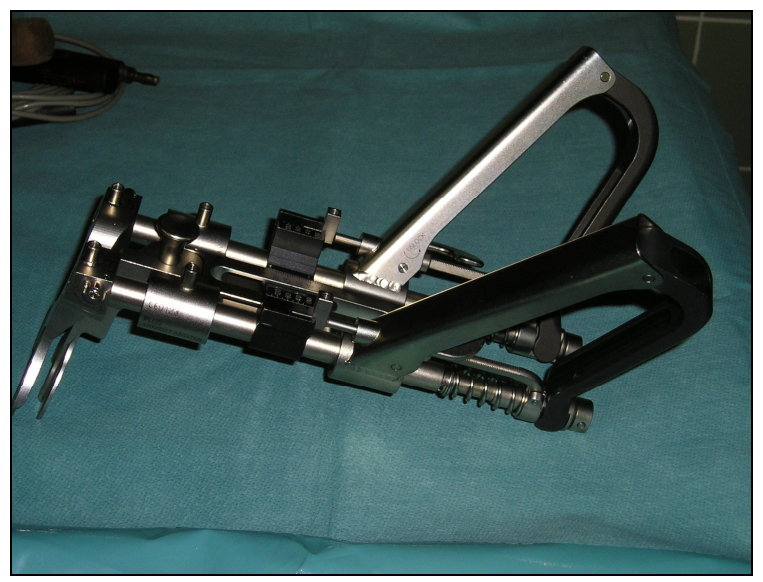

\section{Abbildung 16: Bandspanner}

Zur Optimierung der Rotationsausrichtung der femoralen Implantatkomponente erfolgt nun das veränderte Vorgehen mittels bandspannungsbasierter Navigation. Dazu wird in $90^{\circ}$-Flexion eine mit verschiedenen Stellschrauben versehene Femurgrößenlehre auf der distalen Femurresektionsfläche fixiert. Diese Lehre wird dabei mit Hilfe von zwei Fühlern gegen die dorsalen Kondylen referenziert (siehe Abb. 17).

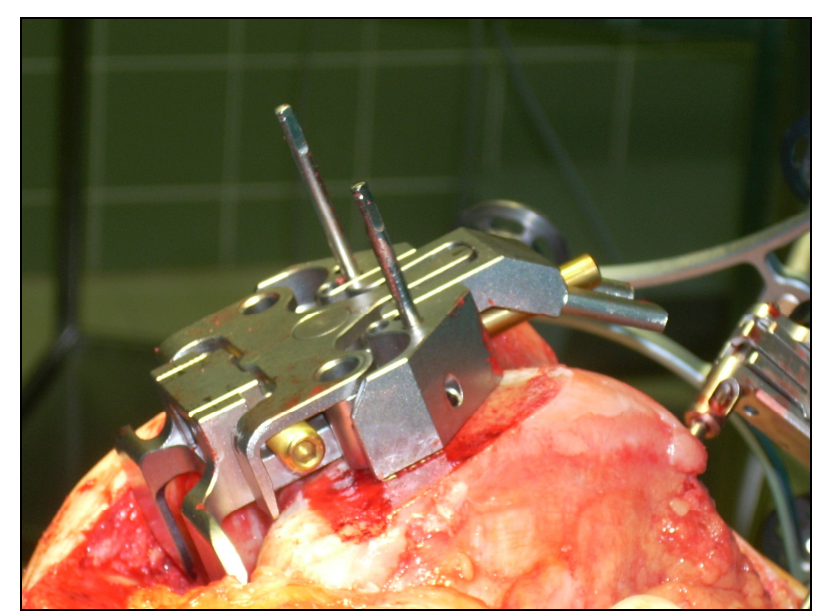

Abbildung 17: Femurgrößenlehre in vivo (aus Walde et al. 2010, Seite 383, mit freundlicher Genehmigung des Elsevier-Verlags)

Anhand eines mit der Größenlehre verbundenen Lokators werden die Femurgrößenlehre durch das Navigationssystem erfasst und alle folgenden Veränderungen registriert. Die Stellschrauben lassen eine Feinjustierung bzgl. der Rotation, Ventral- und Dorsalverschiebung sowie Größenwahl der femoralen Implantatkomponente zu (siehe Abb. 18). Durch den gleichzeitig in den Beugespalt eingesetzten Bandspanner werden die Bänder in Flexion ausbalanciert. 
Mit dem Ziel einen parallelen Beugespalt zu erreichen kann eine Korrektur des Beugespaltes hinsichtlich der Rotation durch die Justierungsmöglichkeit der Lehre bis ca. $6^{\circ}$ erfolgen.

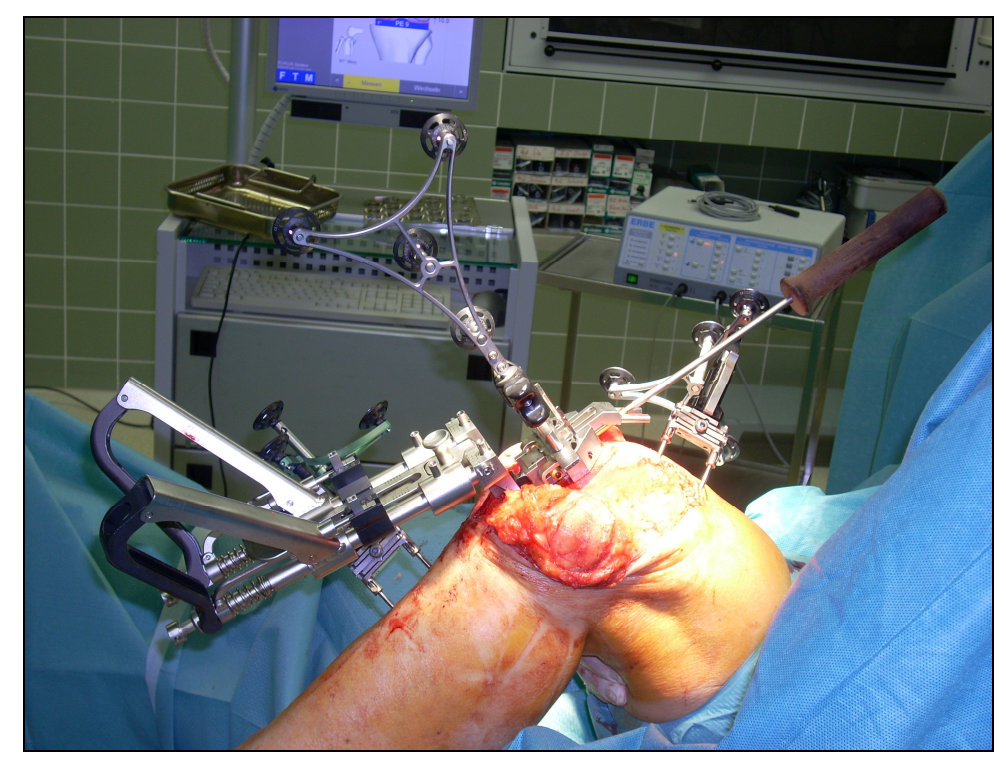

Abbildung 18: Navigationsunterstützte bandspannungsbasierte Festlegung der distalen femoralen Resektionsfläche (aus Walde et al. 2010, Seite 383, mit freundlicher Genehmigung des Elsevier-Verlags)

Nach Bestätigung der Datenregistrierung werden die Bohrlöcher für den 4-in-1Sägeblock (speed block) gefertigt, der Sägeblock aufgesetzt und die vier Resektionen mit der oszillierenden Säge getätigt (siehe Abb. 19). Danach werden weitere Bearbeitungen des Femur wie etwa mittels Trochlea-Meißel vorgenommen.

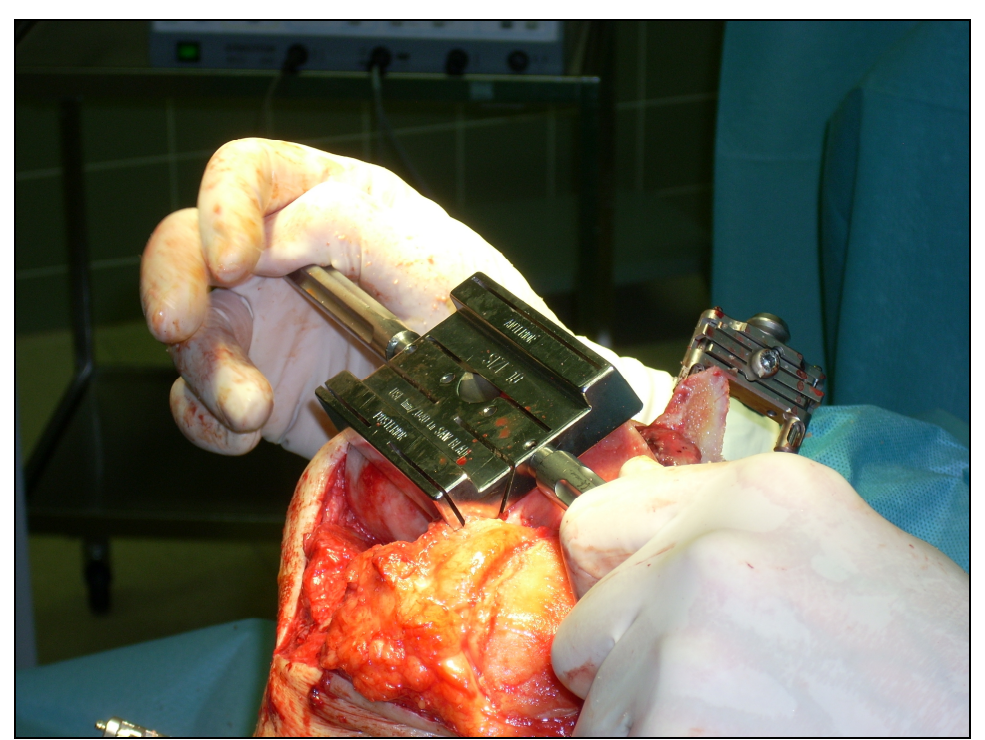

Abbildung 19: Positionierung des 4-in-1-Sägeblocks (speed block) in vivo 
Nach erfolgter Femurresektion wird der idealerweise parallele Resektionsspalt zum Abschluss jeweils in Extension und Flexion unter Berücksichtigung der Bandspannung mit dem Bandspanner zur Bestimmung des PE-Inlays gemessen (siehe Abb. 20 und 21). Die aufzuwendende Kraft wird in Newton pro Kondyle angezeigt und beträgt wieder 90 Newton.

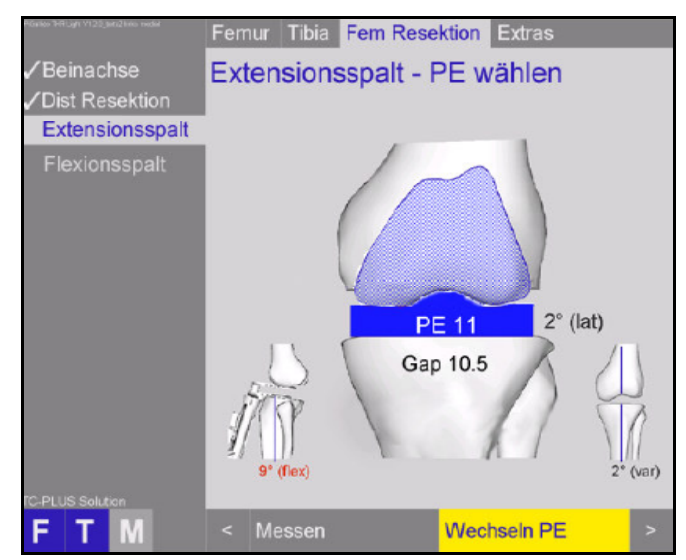

Abbildung 20: Monitorbild - Wahl des PE-Inlays und Messung des Resektionsspalts (aus Operationsanleitung PiGalileo TKR Light ${ }^{\circledR}$ 2006, Seite 37, mit freundlicher Genehmigung der Smith \& Nephew GmbH)

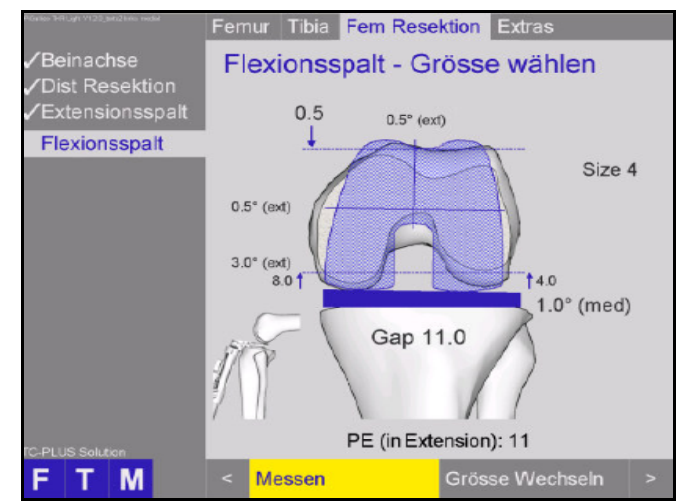

Abbildung 21: Rotations- und Größenbestimmung der Femurkomponente unter Berücksichtigung der Bandspannung in Flexion (aus Operationsanleitung PiGalileo TKR Light ${ }^{\circledR}$ 2006, Seite 38, mit freundlicher Genehmigung der Smith \& Nephew GmbH)

Die angezeigten und protokollierten Werte verhalten sich relativ zu den vorher registrierten konventionellen knöchernen Landmarken und sind Gegenstand der Studie. Abschließend wird eine Funktionsanalyse der Probeimplantate mit Aufzeichnung des postoperativen Bewegungsumfanges durchgeführt. 
Die postoperative ROM-Messung wird zur Datenerhebung gespeichert und enthält Informationen bzgl. Varus-/Valgusstellung, Extensions-/Flexionswinkel und Rotationsabweichung bezogen auf die bandspannungsbasierte OP-Technik (siehe Abb. 22). Die registrierten Datensätze wurden nach Abschluss der Studie aus dem Navigationssystem ausgelesen.

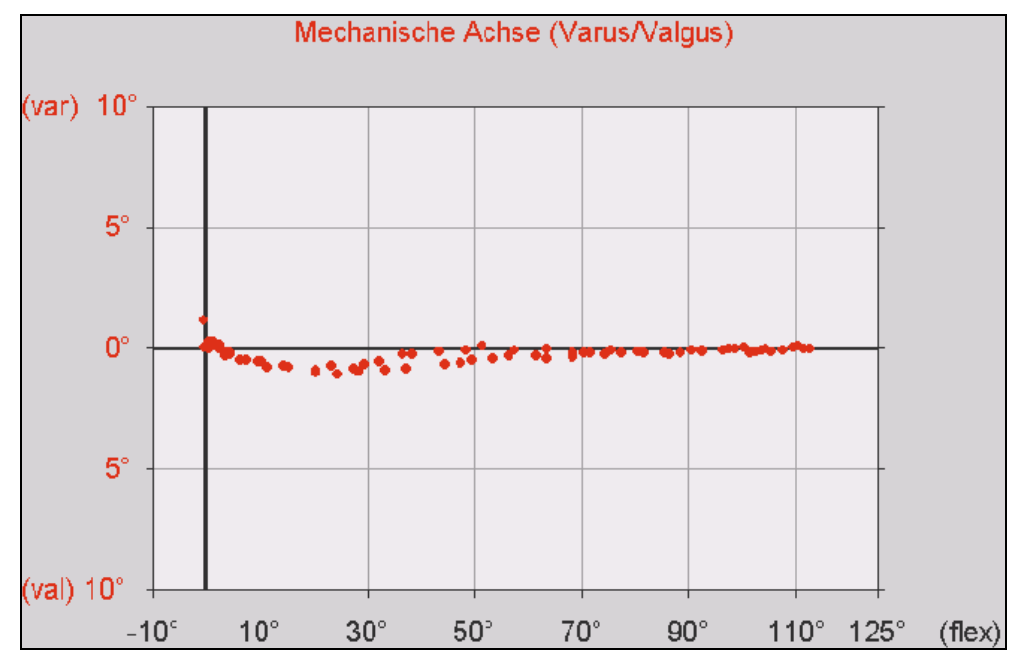

Abbildung 22: Postoperative ROM-Messung (Beispielabbildung eines Studienpatienten)

\subsection{Patienten und Einschlusskriterien}

Ab Juni 2006 bis einschließlich Juli 2007 wurden 93 Patienten geschlechts- und altersunabhängig sowie pseudonymisiert prospektiv in die Studie zur bandspannungsbasierten Rotationsausrichtung der femoralen Implantatkomponente unter Anwendung des Navigationssystems PiGalileo TKR Light ${ }^{\circledR}$, Version 1.3 (Smith \& Nephew $\mathrm{GmbH}$ ) aufgenommen. Bei allen Patienten lag eine klinisch sowie radiologisch diagnostizierte primäre Gonarthrose mit Indikation und Wunsch zum endoprothetischen Ersatz des Kniegelenks vor.

In allen Fällen erfolgte die zementfreie (press fit) oder zementierte Implantation einer bi- bzw. trikompartimentellen Kniegelenksprothese $\left(\mathrm{EFK}^{\circledR}\right.$-Prothese, Smith \& Nephew $\mathrm{GmbH}$ ) mit der Studiennavigation.

Vor Beginn wurde das Studiendesign mit folgenden Einschlusskriterien festgelegt: 
1. Vorliegen einer präoperativ klinisch weitestgehend korrigierbaren intraartikulären Fehlstellung unter Varus-/Valgusstress. Nach Abtragung der Osteophyten und nach Anlage der Lokatoren erfolgte eine intraoperative Kontrolle der Korrigierbarkeit (Walde et al. 2010).

2. Vorliegen einer präoperativen Varus-/Valgusabweichung von maximal $15^{\circ}$ bezogen auf die mechanische Achse in der a.p.-Ganzbeinstandaufnahme (Walde et al. 2010).

Die zur Studienteilnahme berechtigten Patienten gaben vor Studienbeginn eine Einwilligungserklärung ab. Im Rahmen dieser Studie kam es zu keiner zusätzlichen Strahlenbelastung für die Patienten. Die durchgeführten Röntgenbilder entsprachen in ihrer Anzahl und Art derer, die routinemäßig zur präoperativen Diagnostik angefertigt werden. Durch den Beschluss der Ethik-Kommission der Universität zu Göttingen mit der Antragsnummer 2/1/08 wurde die Studie bewilligt.

\subsection{Auswertung und Visualisierung der OP-Daten}

Die Grundlage der Datenauswertung bildeten die intraoperativ registrierten Messungen von 93 Patienten, welche von dem Navigationssystem PiGalileo TKR Light $^{\circledR}$, Version 1.3 (Smith \& Nephew GmbH) gespeichert wurden. Die in der Anwendungssoftware enthaltenen mathematischen Berechnungen und die Ansteuerung des Messsystems wurden von der AXIOS 3D ${ }^{\circledR}$ Services $\mathrm{GmbH}$ in Oldenburg, Deutschland entwickelt. Für alle navigierten Implantationen stand die intern verwendete Berechnungsbibliothek Gena Light 1.5.1 zur Verfügung, in der nach jedem Navigationsschritt während der Operation eine vollständig aktualisierte Datei angelegt wurde. Darin waren sämtliche Messwerte in detaillierter Form enthalten, und zwar die kompletten geometrischen 3D-Daten aller knöchernen Messungen und Ausrichtungen. Die Auswertung der OP-Datensätze erfolgte mit dem Programm "Gena Light Interpreter" (AXIOS 3D ${ }^{\circledR}$ Services GmbH). Das Auswertungsprogramm basiert auf den Berechnungsmodulen, die auch in der Studiennavigationssoftware verwendet wurden, wodurch die Berechnungen identisch waren. 
Nach der Implantation mit der navigierten bandspannungsbasierten OP-Technik wurden die intraoperativ protokollierten Dateien eingelesen und durch den "Gena Light Interpreter" (AXIOS 3D ${ }^{\circledR}$ Services $\mathrm{GmbH}$ ) automatisch weiterverarbeitet. Die errechneten Daten wurden von dem Programm als eine einfache Text-Datei herausgegeben. Die Text-Datei enthielt Informationen bzgl. des Differenzwinkels der 3 knöchernen Landmarken zu der Tibiaresektion und des Differenzwinkels der navigierten ventralen Femurausrichtung zu der mittleren ventralen Richtung der knöchernen Landmarken. Zusätzlich wurden der Beugespalt sowie der Patientencode und das OP-Datum ausgegeben. Zur Visualisierung der ermittelten Daten wurde für jeden OP-Datensatz ein VRML-Modell generiert, in dem die knöchernen Landmarken und die relevanten Messungen bzw. die geometrischen Lagen angezeigt wurden (siehe Abb. 23). Die 3 knöchernen Landmarken waren farblich gekennzeichnet: 1.) TEA in grün, 2.) PKA in blau und 3.) Punkte der Whiteside-Linie in gelb. Zudem wurden hinsichtlich der Achsverhältnisse das Hüftrotationszentrum (FRC), der Femurdurchstoßpunkt (FIP) und der Tibiadurchstoßpunkt (TIP) sowie die Richtungen am Femur optisch reproduziert.

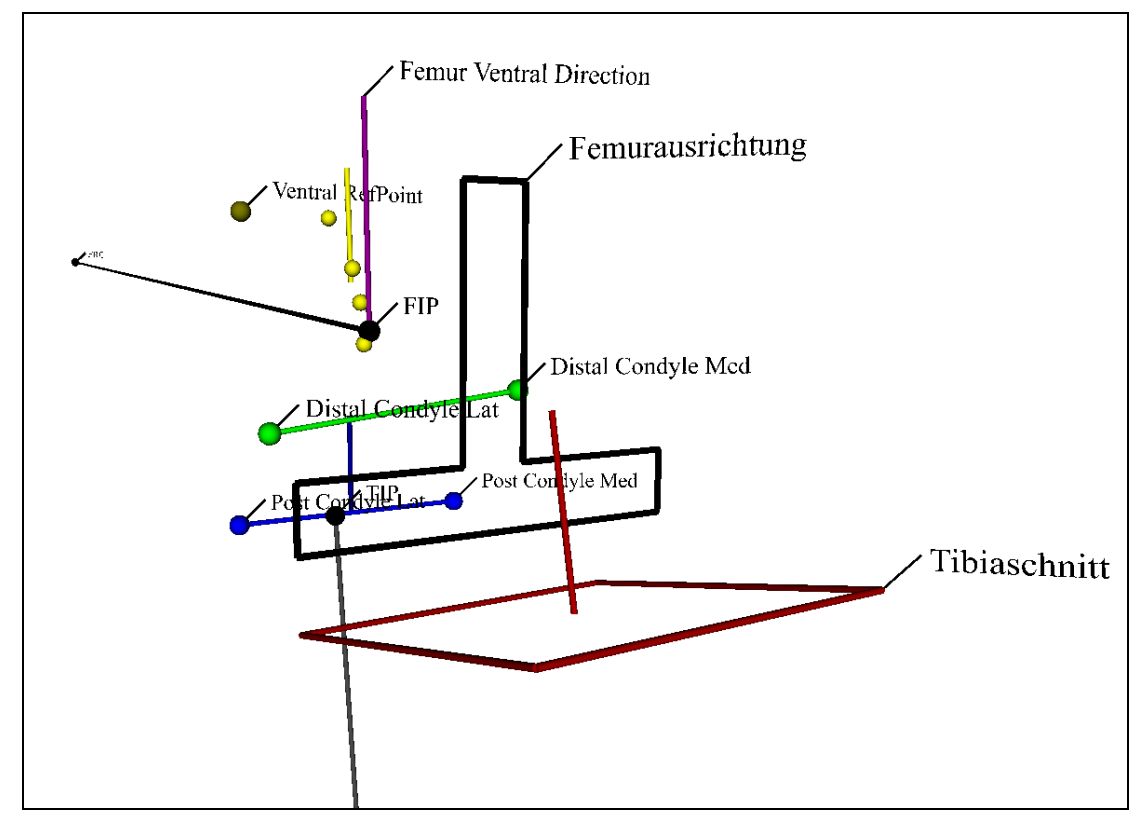

Abbildung 23: Bespielmodell einer VRML-Darstellung, ventrale Richtung der TEA (rot), ventrale Richtung der PKA (blau), ventrale Richtung der Whiteside-Linie (gelb), mittlere ventrale Richtung aus TEA, PKA und Whiteside-Linie (violett), Lage der navigierbaren Femurgrößenlehre (schwarz/gedrehtes " $\mathrm{T}$ “), Lage der registrierten Tibia-Resektionsebene beim Speichern des Beugespalts (rot) 


\subsubsection{Erstellung von Funktionsdiagrammen in Abhängigkeit der knöchernen Landmarken}

Für alle durchgeführten Operationen mit der Studiennavigation wurden die Funktionsdiagramme vor und nach erfolgter Implantation der Probeimplantate protokolliert und präsentierten den Bewegungsumfang des Kniegelenks bzgl. der Extension und Flexion (ROM) mit jeweiliger Varus- bzw. Valgusstellung (siehe Abb. 24).

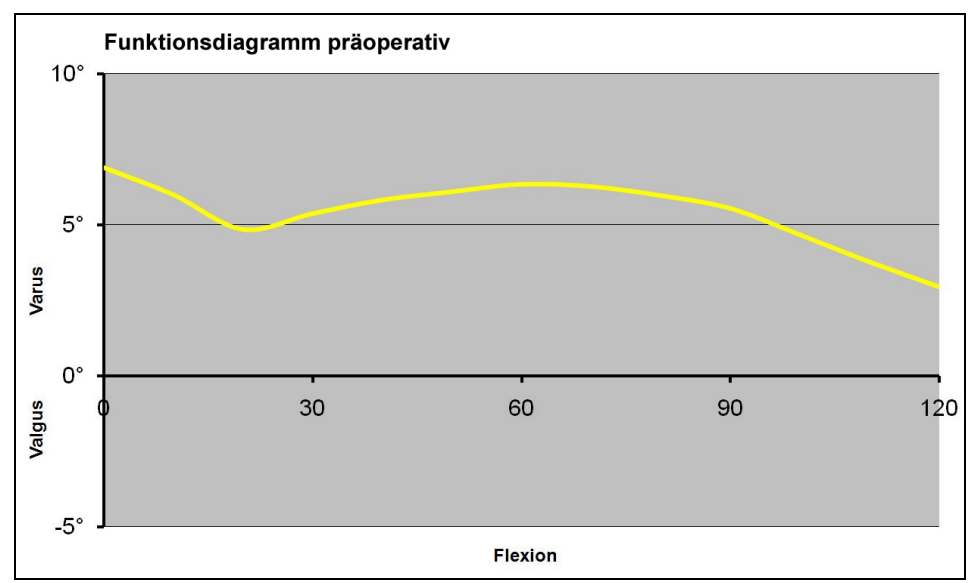

Abbildung 24: Beispiel der kinematischen Achsabweichung der Beinachse, präoperative Messung (aus Walde et al. 2010, Seite 382, mit freundlicher Genehmigung des Elsevier-Verlags)

Unter Anwendung des „Gena Light Interpreters“ (AXIOS 3D ${ }^{\circledR}$ Services $\mathrm{GmbH}$ ) war der Winkel der ventralen Femurausrichtung, d. h. der navigierten bandspannungsbasierten Rotationsausrichtung zu der mittleren ventralen Richtung der 3 knöchernen Landmarken bekannt. Anhand dieser Daten konnten entsprechende Funktionsdiagramme in Abhängigkeit $\mathrm{zu}$ den unterschiedlichen femoralen Rotationsausrichtungen virtuell berechnet werden. Zur Berechnung des jeweiligen Funktionsdiagramms bezogen auf die intraoperativ registrierten knöchernen Landmarken musste $\mathrm{zu}$ den ermittelten ROM-Werten ein Korrekturwert addiert werden, der durch eine Sinusfunktion errechnet wurde.

Korrekturwert $=$ Sinus(Flexionswinkel) ${ }^{*}$ Rotationsunterschied Femurausrichtung zu knöchernen Landmarken 
Dadurch konnten nun postoperative Funktionsdiagramme in Abhängigkeit der verschiedenen knöchernen Rotationsausrichtungen graphisch dargestellt und miteinander verglichen werden. Zusätzlich wurde wie oben beschrieben ein postoperatives Funktionsdiagramm mit Ausrichtung der femoralen Komponente unter bandspannungsbasierter Navigation angefertigt. Somit standen inklusive des präoperativen Funktionsdiagramms insgesamt fünf Funktionsdiagramme von jedem Patienten für die weitere Auswertung zur Verfügung (siehe Abb. 25).

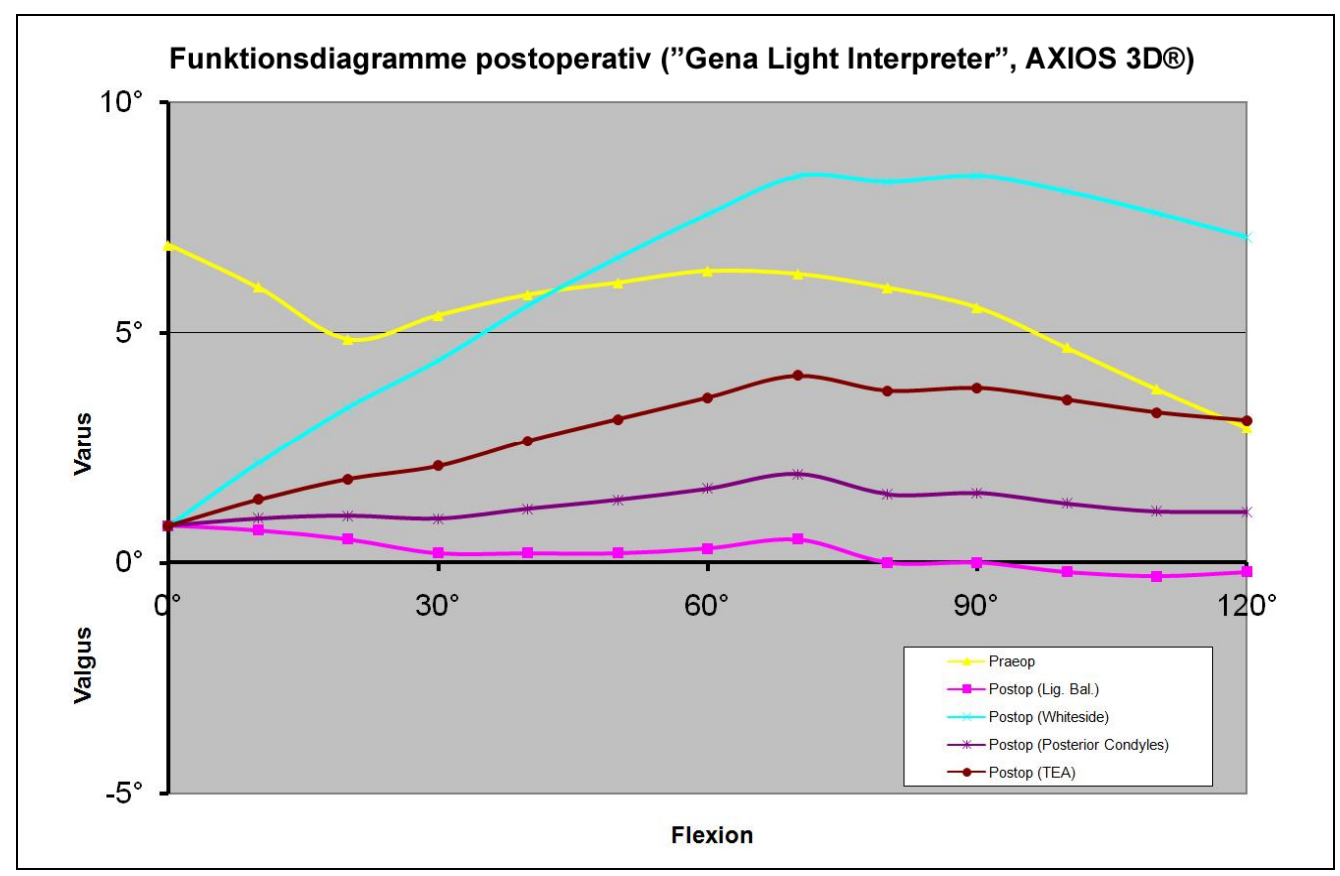

Abbildung 25: ROM-Messung im Vergleich der jeweiligen femoralen Rotationsausrichtung (aus Walde et al. 2010, Seite 383, mit freundlicher Genehmigung des ElsevierVerlags)

\subsubsection{Definition und Berechnung Winkelabweichung}

Aus den Kurven der Funktionsdiagramme wurde die dazugehörige Winkelabweichung von $0^{\circ}$ über die dargestellte Flexionsspanne als Maßzahl der Gelenkfunktion festgelegt und in der Einheit "Grad“ $\left({ }^{\circ}\right)$ angegeben (siehe Abb. 26) (Walde et al. 2010). Dieser Winkel war als Maß der Abweichung in Varus-I Valgusrichtung über die gemessene Flexionsspanne definiert (Walde et al. 2010). Ein Flexionsbereich von zum Beispiel $10^{\circ}$ bis $110^{\circ}$ entspricht einer Flexionsspanne von $100^{\circ}$, wobei positive Werte eine Achsdeviation in Varusrichtung und negative Werte dementsprechend eine Valgusabweichung darstellen. 
Es wurde angenommen, dass die Kurvenfläche, d. h. die Fläche zwischen der Kurve und der Nullinie, in Korrelation zur Winkelabweichung stand. Die Einheit der Kurvenfläche war "Grad zum Quadrat" $\left({ }^{\circ}\right)^{2}$. Die Berechnung der Winkelabweichung erfolgte nach folgender Gleichung:

\section{Winkelabweichung $\left({ }^{\circ}\right)=\quad$ Fläche zwischen der Kurve und der Nulllinie $\left(^{\circ}\right)^{2}$ \\ Flexionsspanne $\left({ }^{\circ}\right)$}

Die errechneten Werte waren Gegenstand der weiteren Evaluation, wobei ein Wert gegen Null, d. h. die geringste Abweichung in Varus-/Valgusrichtung, als optimal anzusehen ist.

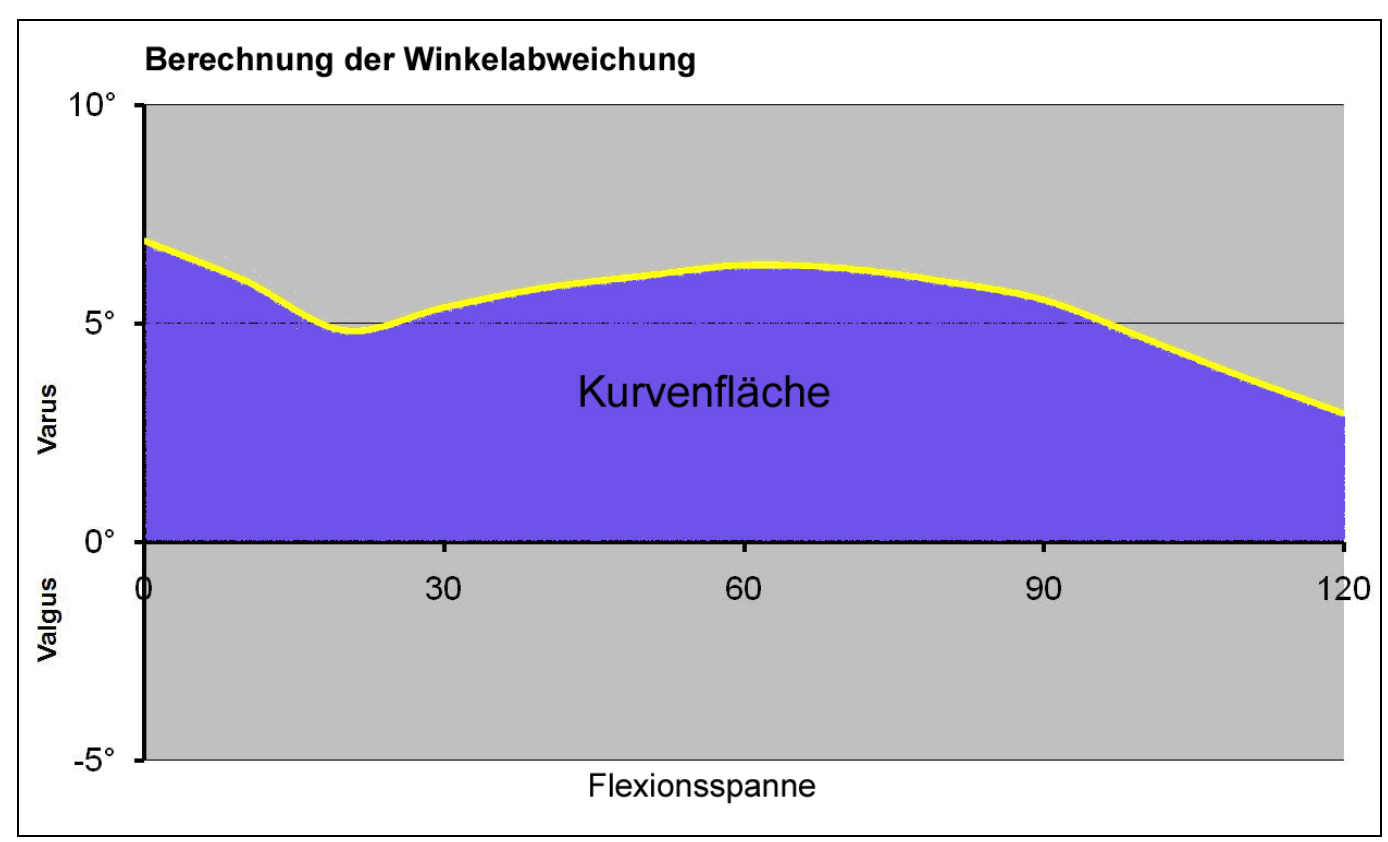

Abbildung 26: Darstellung zur Berechnung der Winkelabweichung (modifiziert aus Walde et al. 2010, Seite 382, mit freundlicher Genehmigung des Elsevier-Verlags)

Des Weiteren wurde, bezogen auf das Funktionsdiagramm der jeweiligen knöchernen Landmarke und der bandspannungsbasierten femoralen Rotationsausrichtung, die jeweilige dynamische Varus- oder Valgusabweichung bei $90^{\circ}$-Flexion zum Vergleich bestimmt. Dabei wurden jeweils die Mittelwerte der absoluten Werte und die maximalen Abweichungen mit dem präoperativen ROMWert bei $90^{\circ}$-Flexion verglichen. 


\subsubsection{Bestimmung der femoralen Rotationsausrichtungen zur tibialen Resektionsfläche beim Beugespalt}

Bei $90^{\circ}$-Flexion wurden die Winkel der Whiteside-Linie, der posterioren Kondylenachse und der transepikondylären Achse in Korrelation zur tibialen Resektionsfläche mit dem Auswertungsprogramm "Gena Light Interpreter" (AXIOS $3 D^{\circledR}$ Services $\mathrm{GmbH}$ ) errechnet (Walde et al. 2010). Dabei wurde auch der Winkel der tibialen Resektionsfläche zur femoralen Rotationsausrichtung mit bandspannungsbasierter Navigation in 90-Flexion ermittelt, um diesen mit den 3 knöchernen Landmarken hinsichtlich eines balancierten Beugespaltes vergleichen zu können. Ein balancierter Beugespalt lag definitionsgemäß bei $0^{\circ}$ vor. Positive Winkelwerte zeigen eine Abweichung in femorale Innenrotation. Demzufolge ist eine Abweichung in femorale Außenrotation durch negative Werte bestimmt. Zum Vergleich wurden die Mittelwerte der absoluten Werte sowie die maximalen Rotationsabweichungen berechnet und in der Einheit „Grad“ $\left(^{\circ}\right)$ dargestellt.

\subsubsection{Statistische Analyse}

Das Tabellenkalkulationsprogramm Microsoft Office Excel 2003 wurde zur Datenerhebung und Dokumentation angewendet. Mit der Statistiksoftware GraphPad PrismTM $^{\circledR}$ (GraphPad Software, San Diego, USA) wurden signifikante Unterschiede mittels univariater (one-way) Varianzanalyse (ANOVA) analysiert. Dabei wurde im Vorfeld des Signifikanztests eine Untersuchung auf Unterschiede der Mittelwerte durchgeführt, um zu ermitteln, ob die Rotationsausrichtungen unterschiedliche Varianzen aufweisen. Dies erfolgte mittels des so genannten Levene-Tests auf Homogenität der Varianzen. Bei anzunehmender Varianzheterogenität musste ein robusteres Testverfahren zur Prüfung der Mittelwertunterschiede angewendet werden (Welch-Test). Im Anschluss wurde ein post-hoc Test durchgeführt. Damit wurde bestimmt, zwischen welchen Rotationsausrichtungen signifikante Unterschiede bzgl. der Mittelwerte bestehen. Als multipler Vergleichstest wurde der Dunnett's multiple comparison post-hoc test (GraphPad Software, San Diego, USA) benutzt. Als signifikant wurden $\mathrm{p}$-Werte $<0.05$ betrachtet. Der Dunnett's test kam zur Anwendung, da von ungleichen Varianzen bei gleicher Stichprobengröße auszugehen war (Janssen und Laatz 2007). Des Weiteren wurde IBM SPSS Statistics (IBM Software, Armonk, USA) zur statistischen Analyse herangezogen. 
Die Illustration der statistischen Auswertungen wurde durch Diagramme und Boxplots vorgenommen. Bei den Boxplots ist der Median als schwarzer, horizontaler Strich in der Fläche der Box eingezeichnet. Die Fläche der Box entspricht dem Bereich, in dem sich die mittleren $50 \%$ der Daten befinden. Durch die $25 \%$ - und 75\%-Perzentile ist die Unter- und Obergrenze, d. h. die Länge der Box dargestellt (Janssen und Laatz 2007). Dieser Bereich, auch Interquartilsabstand genannt, gibt die Ausdehnung der Box an. Durch die dünnen Querstriche unter- und oberhalb der Box, die so genannten Whisker, werden die Werte des Datensatzes dargestellt, die sich noch im Bereich des 1,5-fachen Interquartilsabstands befinden (Janssen und Laatz 2007). Werte darüber werden durch Kreise als Ausreißer markiert. Extreme Ausreißer sind durch Sternchen visualisiert.

Eine Prüfung des Zusammenhangs zwischen Kurvenfläche und Winkelabweichung erfolgte mit dem Korrelationskoeffizienten nach Pearson (Janssen und Laatz 2007). Damit wurde überprüft, ob die Winkelabweichung eine valide Maßzahl für die Kurvenfläche darstellt.

Die Angabe von Mittelwerten (M) bezieht sich auf das arithmetische Mittel. Hierzu wurden alle Werte einer Messreihe addiert und durch die Anzahl der Messwerte dividiert. Das zusätzlich angegebene Konfidenzintervall bezieht sich auf die 95\%Vertrauenswahrscheinlichkeit. Bei dieser so genannten Intervallschätzung wird ein Bereich berechnet, in dessen Grenzen mit einer 95\%-igen Wahrscheinlichkeit der Mittelwert liegt (Janssen und Laatz 2007). Die Standardabweichung (SD) entspricht der Quadratwurzel der Varianz und ist ein Maß für die Streuung der Einzelwerte um ihren Mittelwert, wodurch die Streuung veranschaulicht wird (Koschack 2008).

Alle Ergebnisse der statistischen Auswertung wurden symmetrisch auf die 1. bzw. 2. Dezimalstelle gerundet.

Eine Überarbeitung der Graphiken erfolgte mit dem Programm Adobe ${ }^{\circledR}$ Photoshop ${ }^{\circledR} 7.0$ (Adobe Systems, San Jose, USA). Da es sich in der vorliegenden Arbeit um eine deskriptive Untersuchung handelt, waren die Funktionen der angegebenen EDV-Programme zur statistischen Berechnung ausreichend. 


\section{Ergebnisse}

\subsection{Winkelabweichung und Achsdeviation bei Auswertung der} Funktionsdiagramme in Abhängigkeit der femoralen Rotationsausrichtung

Die präoperative ROM-Evaluation der mechanischen Beinachse in Streckstellung zeigt bei $\mathrm{n}=93$ eine mittlere Achsabweichung von $3,9^{\circ}$ Varus. Die maximale Abweichung in Varusrichtung beträgt $11,2^{\circ}$. Eine maximale Deviation in Richtung Valgus ist bis $3,1^{\circ}$ zu eruieren. Bei $n=16$ liegt ein Streckdefizit von $10^{\circ}$ vor. In einem Fall $(n=1)$ besteht ein präoperatives Streckdefizit von $20^{\circ}$. Vor Studienbeginn ist bei 81 Patienten eine Varusfehlstellung und bei 12 Patienten eine Valgusfehlstellung diagnostiziert worden (siehe Abb. 27). Insgesamt bedeutet dies, dass in $87 \%$ der Fälle eine Varusgonarthrose die Indikation zur endoprothetischen Versorgung darstellt.

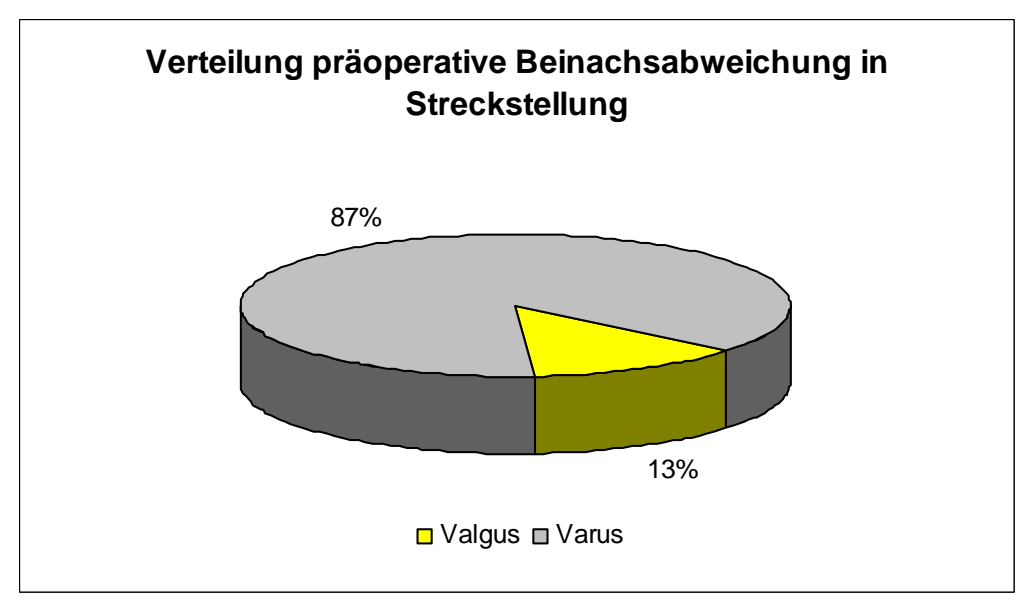

Abbildung 27: Diagramm präoperative Beinachsabweichung bei $0^{\circ}$

Bei der deskriptiven Analyse der Funktionsdiagramme hinsichtlich der Kurvenfläche zeigt sich ein deutlich geringerer Mittelwert bei der Rotationsausrichtung der femoralen Implantatkomponente mit bandspannungsbasierter Navigation (kurz: bandspannungsbasierte Femurausrichtung) im Vergleich zu den knöchernen Landmarken sowie präoperativ (siehe Tab. 2). Mit Blick auf die Maßzahlen der Streuung des Mittelwertes (min., max. und SD) wird auch eine geringere Varianz bei der bandspannungsbasierten Femurausrichtung deutlich. 
Mit einem 95\%-Konfidenzintervall des Mittelwerts von $\left[111,8\left({ }^{\circ}\right)^{2} ; 141,2\left({ }^{\circ}\right)^{2}\right]$ hat die bandspannungsbasierte Femurausrichtung vor der PKA das geringste Ausmaß. Die Schwankungsbreite ist bei der Whiteside-Linie am größten, so dass die zufällige Abweichung vom Mittelwert hierbei am höchsten ist. Die posteriore Kondylenachse hat zwar den zweitkleinsten Mittelwert, weist jedoch eine höhere Standardabweichung im Vergleich zur TEA auf. Die maximal berechnete Kurvenfläche der Whiteside-Linie ist dem maximalen präoperativen Wert von $1272\left({ }^{\circ}\right)^{2}$ sehr nahe, wobei der minimale Wert fast identisch ist mit der bandspannungsbasierten Femurausrichtung.

\begin{tabular}{|c|c|c|c|c|c|}
\hline & \multirow{2}{*}{$\begin{array}{c}\text { präoperative } \\
\text { Kurvenfläche } \\
\qquad\left({ }^{\circ}\right)^{2}\end{array}$} & \multicolumn{4}{|c|}{ postoperative Kurvenfläche $\left({ }^{\circ}\right)^{2}$} \\
\hline & & $\begin{array}{l}\text { Whiteside- } \\
\text { Linie }\end{array}$ & PKA & TEA & $\begin{array}{c}\text { bandspannungsbasierte } \\
\text { Femurausrichtung }\end{array}$ \\
\hline Mittelwert & 506,6 & 367,1 & 247,5 & 273,4 & 126,5 \\
\hline $\min$. & 98 & 20 & 28 & 47 & 19 \\
\hline $\max$. & 1272 & 1091 & 905 & 785 & 339 \\
\hline SD & 248,23 & 246,59 & 166,86 & 155,59 & 70,22 \\
\hline $\mathrm{Cl}$ & {$[454,6 ; 558,6]$} & $\begin{array}{l}{[315,4 ;} \\
418,7]\end{array}$ & $\begin{array}{l}{[212,6 ;} \\
282,5]\end{array}$ & $\begin{array}{l}{[223,6 ;} \\
323,2]\end{array}$ & {$[111,8 ; 141,2]$} \\
\hline
\end{tabular}

Tabelle 2: Vergleich der Kurvenflächen

Der Boxplot präsentiert, dass bei allen berechneten Kurvenflächen Ausreißer auftreten. Die größte Streuung unter den 4 Verfahren zur Rotationsausrichtung der Femurkomponente tritt bei der Whiteside-Linie auf (siehe Abb. 28). Es zeigt sich, dass die bandspannungsbasierte Femurausrichtung stabiler gegen höhergradige Ausreißer ist, da die Ausreißer nahe dem oberen Whisker liegen. Zudem ist der Interquartilsabstand zur Darstellung der Streubreite bei der bandspannungsbasierten Femurausrichtung sehr eng. Die Einheit der Werte auf der y-Achse, sprich der Kurvenfläche, ist „Grad zum Quadrat“. 


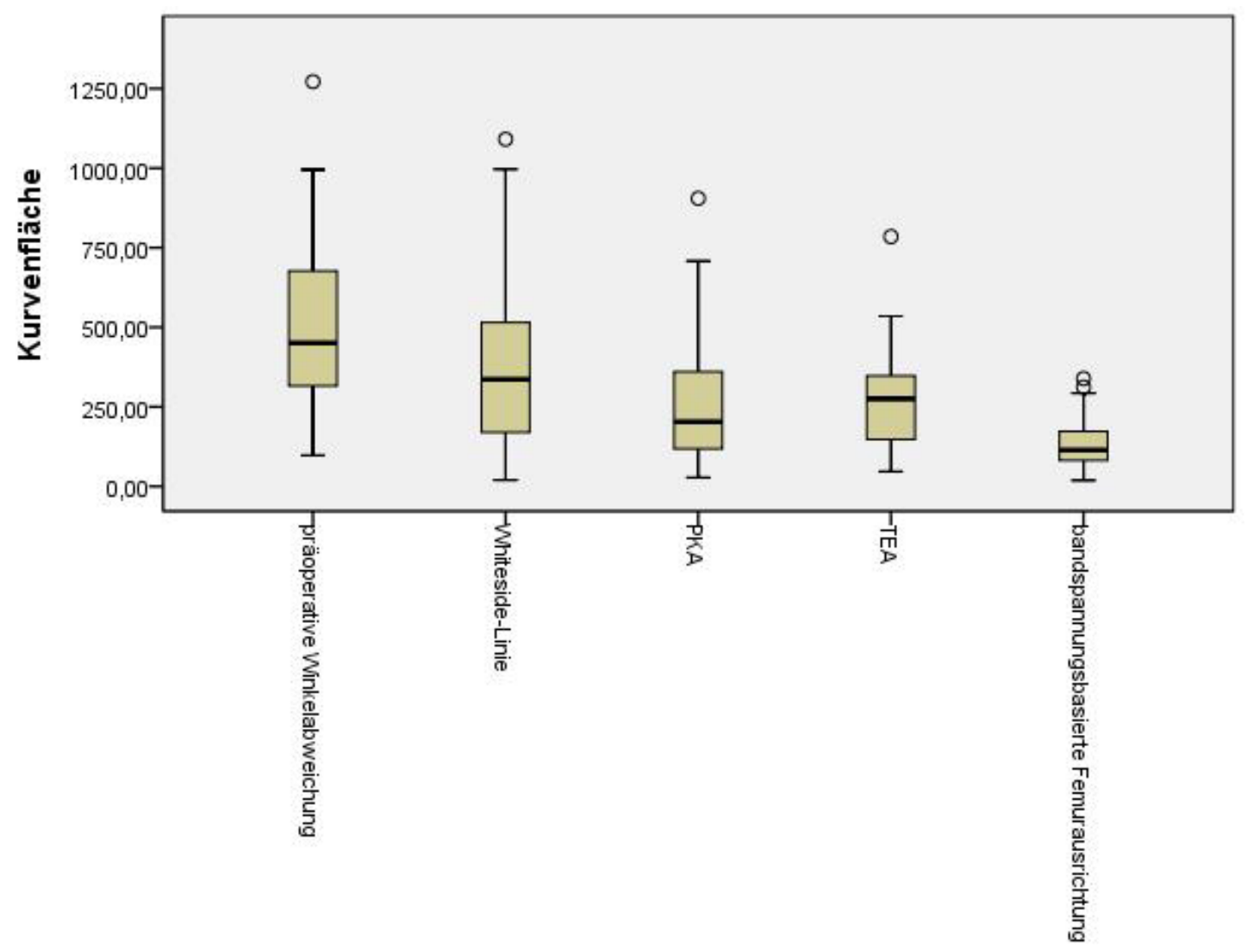

Abbildung 28: Boxplot mit Darstellung der Kurvenfläche (Einheit $\left.\left({ }^{\circ}\right)^{2}\right)$

Die Auswertung der Funktionsdiagramme mit der univariaten Varianzanalyse ergibt mit $p<0.001$ eine signifikante Reduzierung der mittleren Fläche zwischen der Kurve und der Nullinie bei Anwendung der 3 knöchernen Landmarken und der bandspannungsbasierten Femurausrichtung im Vergleich zu der präoperativen Flächenberechnung. Die bandspannungsbasierte Femurausrichtung zeigt hier mit einer mittleren Flächenermittlung von $126,5\left(^{\circ}\right)^{2}$ die größte Differenz im Vergleich zum mittleren präoperativen Wert von $506,6\left({ }^{\circ}\right)^{2}$. Zudem besteht mit $p<0.01$ eine statistisch signifikante Verkleinerung der mittleren Kurvenfläche mit der bandspannungsbasierten Femurausrichtung im Vergleich zu den knöchernen Landmarken.

Eine Korrelation zwischen der Kurvenfläche und der Winkelabweichung als Maßzahl der Gelenkfunktion ist durch den Korrelationskoeffizienten nach Pearson untersucht worden. Bereits das Punktdiagramm (siehe Abb. 29) zeigt eine deutliche Korrelation zwischen der Winkelfläche und der Flächenberechnung zwischen Nullinie und Kurve. Mit einem Korrelationskoeffizienten von 0.997 zeigt sich ein nahezu perfekter Zusammenhang, wobei die Korrelation auf dem Niveau von $p<0.01$ signifikant ist. 


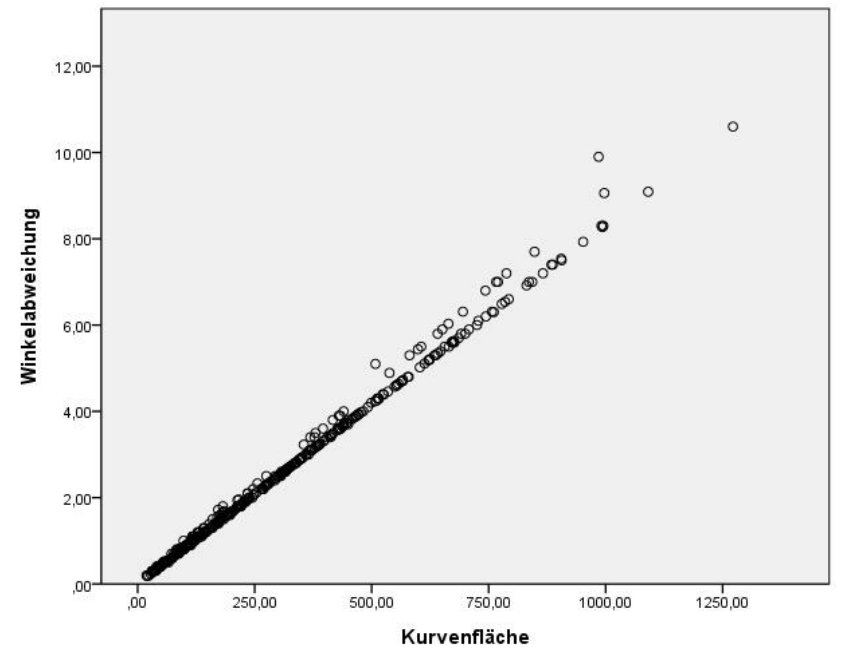

Abbildung 29: Korrelation zwischen Kurvenfläche und Winkelabweichung

Durch die Berechnung der Winkelabweichungen werden die Ergebnisse besser veranschaulicht. Hier zeigt sich mit einem Mittelwert von $1,1^{\circ}$ bei der bandspannungsbasierten Femurausrichtung die höchste Differenz bezogen auf die präoperative mittlere Winkelabweichung von $4,3^{\circ}$ (siehe Tab. 3). Außerdem werden nun die maximalen Winkelabweichungen übersichtlicher. Auch hierbei stellt sich die Methode mit der bandspannungsbasierten Studiennavigation zur femoralen Rotationsausrichtung als diejenige heraus, die sich am ehesten dem Nullwert nähert.

\begin{tabular}{|c|c|c|c|c|c|}
\hline & \multirow{2}{*}{$\begin{array}{c}\text { präoperative } \\
\text { Winkelab- } \\
\text { weichung }\end{array}$} & \multicolumn{4}{|c|}{ postoperative Winkelabweichung } \\
\hline & & $\begin{array}{l}\text { Whiteside- } \\
\text { Linie }\end{array}$ & PKA & TEA & $\begin{array}{c}\text { bandspannungsbasierte } \\
\text { Femurausrichtung }\end{array}$ \\
\hline Mittelwert & $4,3^{\circ}$ & $3,1^{\circ}$ & $2,1^{\circ}$ & $2,3^{\circ}$ & $1,1^{\circ}$ \\
\hline $\min$. & $0,8^{\circ}$ & $0,2^{\circ}$ & $0,2^{\circ}$ & $0,4^{\circ}$ & $0,2^{\circ}$ \\
\hline $\max$. & $10,6^{\circ}$ & $9,1^{\circ}$ & $7,5^{\circ}$ & $6,5^{\circ}$ & $2,8^{\circ}$ \\
\hline SD & $2,15^{\circ}$ & $2,09^{\circ}$ & $1,4^{\circ}$ & $1,29^{\circ}$ & $0,58^{\circ}$ \\
\hline $\mathrm{Cl}$ & {$\left[3,9^{\circ} ; 4,8^{\circ}\right]$} & {$\left[2,7^{\circ} ; 3,5^{\circ}\right]$} & {$\left[1,8^{\circ} ; 2,4^{\circ}\right]$} & {$\left[1,9^{\circ} ; 2,7^{\circ}\right]$} & {$\left[0,9^{\circ} ; 1,2^{\circ}\right]$} \\
\hline
\end{tabular}

Tabelle 3: Vergleich der Funktionsdiagramme durch Bestimmung der Winkelabweichung

Bei der Analyse der Winkelabweichung hat sich mit $1,1^{\circ}\left(\mathrm{SD}=0,58^{\circ}\right)$ die geringste mittlere Winkelabweichung bei der bandspannungsbasierten Femurausrichtung (min. $\left.0,2^{\circ} / \max .2,8^{\circ}\right)$ gezeigt. 
Bezogen auf die Reduktion der Winkelabweichung besteht mit $\mathrm{p}<0.01$ eine statistische Signifikanz gegenüber der Whiteside-Linie, der PKA und der TEA. Die posteriore Kondylenachse $\left(\min .0,2^{\circ} / \max .7,5^{\circ}\right)$ weist mit $2,1^{\circ} \quad\left(S D=1,4^{\circ}\right)$ die zweitkleinste mittlere Winkelabweichung auf. Durch die Whiteside-Linie (min. 0,2\% max. $\left.9,1^{\circ}\right)$ wird die mittlere Winkelabweichung mit $3,1^{\circ}\left(S D=2,09^{\circ}\right)$ am wenigsten verringert und die größte postoperative Streuung ausgelöst (siehe Abb. 30). Bei allen 4 Verfahren (Whiteside-Linie, PKA, TEA und bandspannungsbasierte Femurausrichtung) wird mit $p<0.001$ eine statistisch signifikante Abnahme der Winkelabweichung im Vergleich zum präoperativen Wert erreicht. Im Boxplot haben die Werte der Winkelabweichung die Einheit „Grad“.

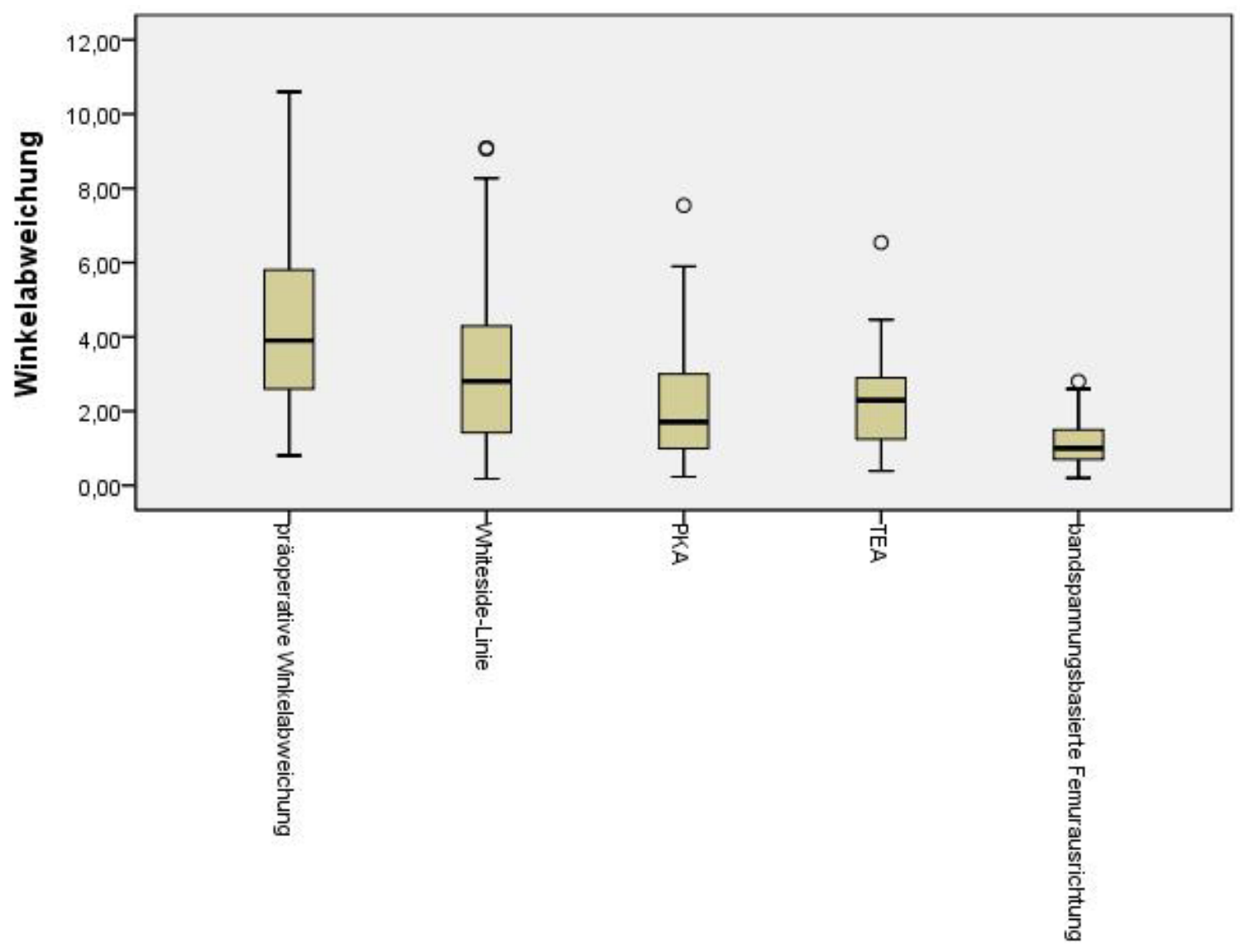

Abbildung 30: Boxplot mit Darstellung der Winkelabweichung (Einheit ${ }^{\circ}$ )

Im Säulendiagramm werden die errechneten Mittelwerte der Winkelabweichungen in Abhängigkeit der Funktionsdiagramme übersichtlich zusammengefasst und der Unterschied der jeweiligen Methode zur Rotationsausrichtung verdeutlicht (siehe Abb. 31). 


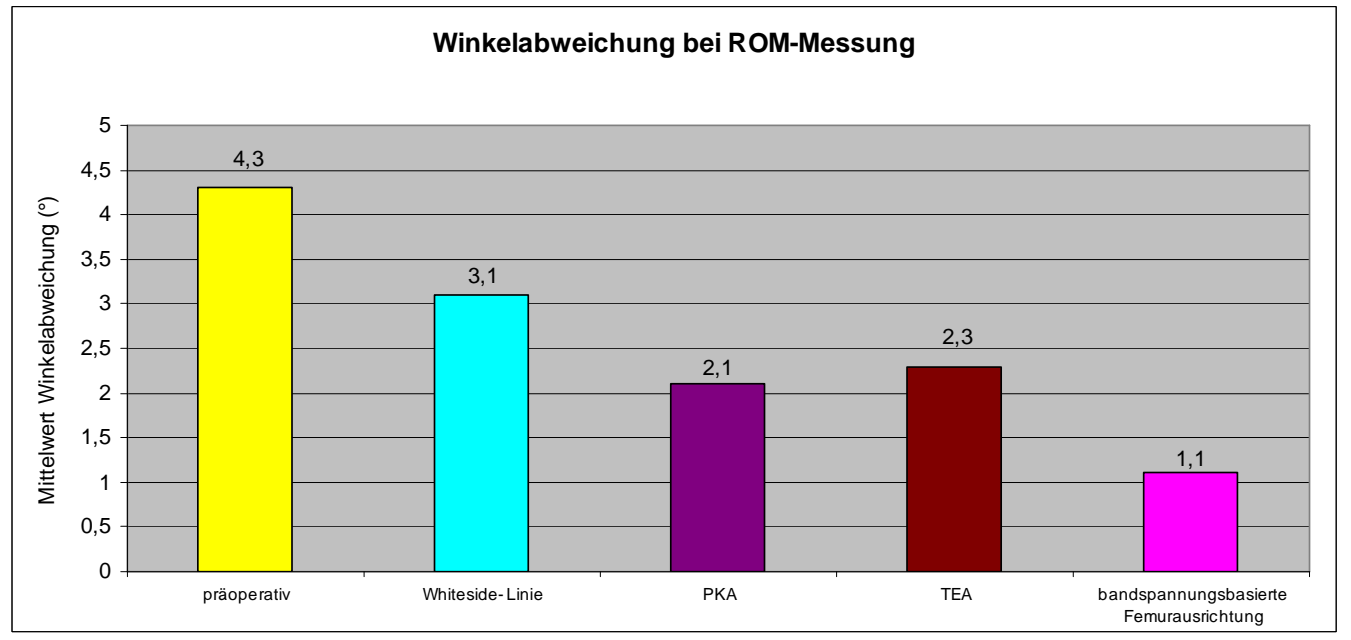

Abbildung 31: Vergleich der mittleren Winkelabweichungen aus den Funktionsdiagrammen

Zur weiteren Evaluation werden die dynamischen Beinachsverhältnisse bei $90^{\circ}$ Flexion betrachtet. Hinsichtlich der Mittelwerte der absoluten Winkelabweichung in Varus-/Valgusrichtung bei $90^{\circ}$-Flexion wird mit $4,0^{\circ}\left(\mathrm{SD}=3,01^{\circ}\right)$ der höchste Wert bei der Whiteside-Linie sichtbar (siehe Tab. 4). Hier ist im Vergleich zur maximalen präoperativen Varusabweichung von 10,4 eine Zunahme der Achsabweichung in Richtung Varus auf $12,8^{\circ}$ zu verzeichnen. Dies entspricht einer Differenz von 2,4 Bei der PKA (maxVar 9,1\%/maxVal $\left.2,7^{\circ}\right)$, der TEA (maxVar $\left.7,7^{\circ} / \operatorname{maxVal} 1,2^{\circ}\right)$ und der bandspannungsbasierten Femurausrichtung (maxVar $\left.3,1^{\circ} / \operatorname{maxVal} 3,3^{\circ}\right) \quad$ wird insgesamt eine Verringerung der Achsdeviation erzielt. Mit einem Mittelwert der absoluten Abweichung von $1,2^{\circ}(S D=0,86)$ und $p<0.001$ liegt bei der bandspannungsbasierten Femurausrichtung eine signifikante Reduzierung der Achsabweichung im Vergleich zu den präoperativen Messwerten und den 3 knöchernen Landmarken vor. Eine Abnahme der Achsabweichung wird mit einem Mittelwert der absoluten Achsabweichung von 2,6 $\left(\mathrm{SD}=1,91^{\circ}\right)$ bei der posterioren Kondylenachse aufgezeigt und ist mit $\mathrm{p}<0.05$ statistisch signifikant verglichen mit der präoperativen Beinachsdeviation. Durch die Whiteside-Linie und die transepikondyläre Achse wird mit $p>0.05$ keine statistisch signifikante Reduktion der Beinachsabweichung erreicht. Insgesamt ist die geringste Standardabweichung und der geringste Korridor der Varus-/Valgusabweichung bei der bandspannungsbasierten Femurausrichtung zu finden (siehe Tab. 4). Hier zeigt zudem das 95\%Konfidenzintervall das geringste Ausmaß. 


\begin{tabular}{|c|c|c|c|c|c|}
\hline & \multirow{2}{*}{$\begin{array}{c}\text { präoperative } \\
\text { Beinachse } \\
90^{\circ}\end{array}$} & \multicolumn{4}{|c|}{ postoperative Beinachse $90^{\circ}$} \\
\hline & & $\begin{array}{l}\text { Whiteside- } \\
\text { Linie }\end{array}$ & PKA & TEA & $\begin{array}{c}\text { bandspannungsbasierte } \\
\text { Femurausrichtung }\end{array}$ \\
\hline $\begin{array}{l}\text { Mittelwert } \\
\text { absolute } \\
\text { Abweichung }\end{array}$ & $3,5^{\circ}$ & $4,0^{\circ}$ & $2,6^{\circ}$ & $2,9^{\circ}$ & $1,2^{\circ}$ \\
\hline maxVar & $10,4^{\circ}$ & $12,8^{\circ}$ & $9,1^{\circ}$ & $7,7^{\circ}$ & $3,1^{\circ}$ \\
\hline maxVal & $7,5^{\circ}$ & $3,2^{\circ}$ & $2,7^{\circ}$ & $1,2^{\circ}$ & $3,3^{\circ}$ \\
\hline SD & $2,28^{\circ}$ & $3,01^{\circ}$ & $1,91^{\circ}$ & $1,31^{\circ}$ & $0,86^{\circ}$ \\
\hline $\mathrm{Cl}$ & {$\left[3,1^{\circ} ; 4,0^{\circ}\right]$} & {$\left[3,4^{\circ} ; 4,6^{\circ}\right]$} & {$\left[2,3^{\circ} ; 3,1^{\circ}\right]$} & {$\left[2,6^{\circ} ; 3,1^{\circ}\right]$} & {$\left[1,0^{\circ} ; 1,4^{\circ}\right]$} \\
\hline
\end{tabular}

Tabelle 4: Dynamische Beinachsabweichungen bei $90^{\circ}$-Flexion

Die Einheit der dargestellten Werte im folgenden Boxplot ist „Grad“. Positive Werte im Boxplot kennzeichnen eine Abweichung in Richtung Varus und negative Werte dementsprechend eine Valgusabweichung. Der Boxplot zeigt, dass bei Ermittlung der Beinachsabweichung bei $90^{\circ}$-Flexion nur die bandspannungsbasierte Femurausrichtung und die TEA keine Ausreißer hervorrufen. Extreme Ausreißer treten bei dieser Analyse nicht auf. Die größte Streuung bei den Verfahren zur Rotationsausrichtung der Femurkomponente ist bei der Whiteside-Linie zu sehen. Des Weiteren wird bei der Whiteside-Linie anhand des oberen Whiskers eine Varuszunahme der Beinachsdeviation im Vergleich zur präoperativen oberen Whiskerlänge offensichtlich. Der kleinste Interquartilsabstand ist bei der bandspannungsbasierten Femurausrichtung zu finden.

Zusammenfassend lässt sich aus dem Boxplot ableiten, dass dieses Verfahren zur femoralen Rotationsausrichtung die stabilste Methode mit der geringsten Streuung ist. 


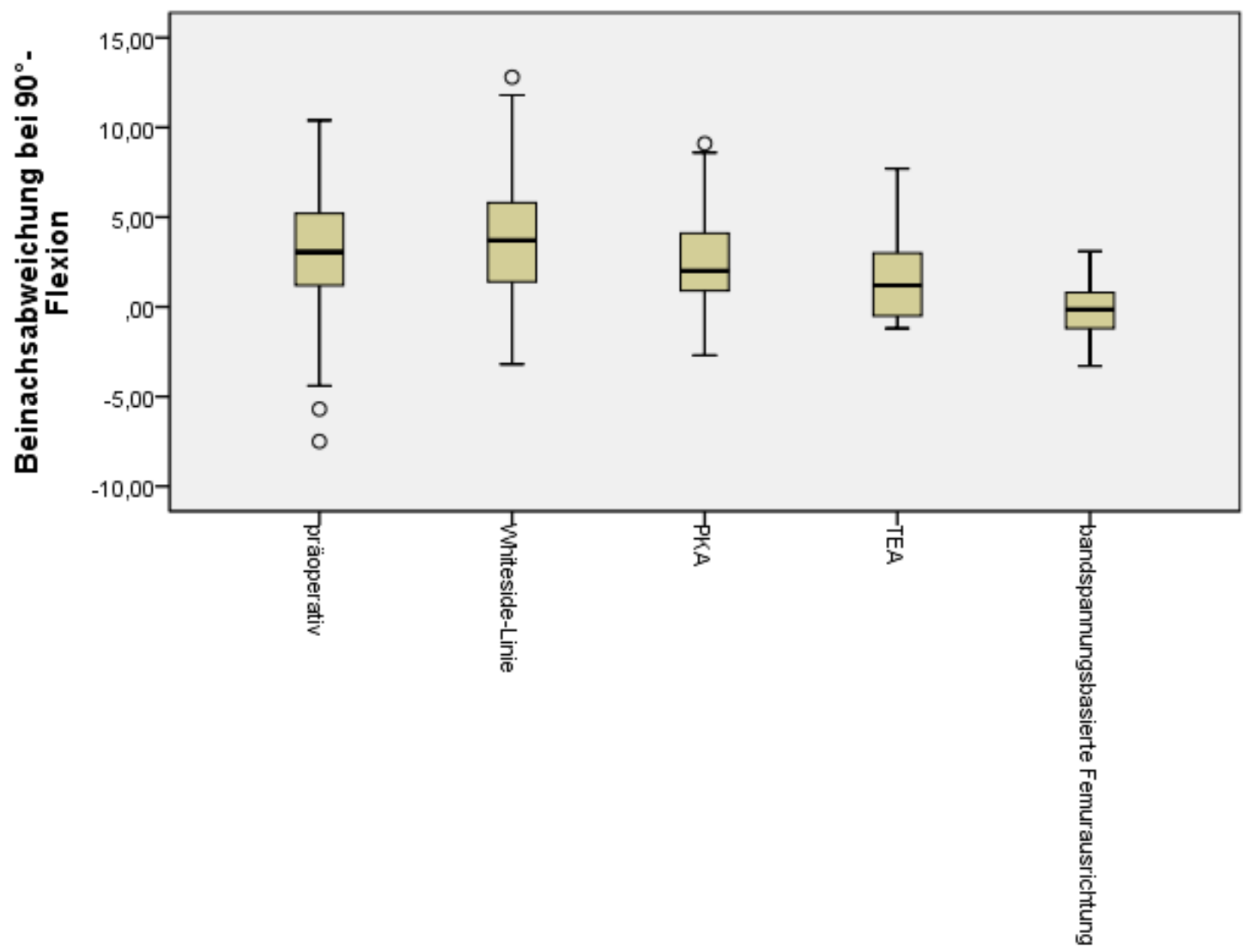

Abbildung 32: Boxplot zur Darstellung der Beinachsabweichung bei $90^{\circ}$-Flexion (Einheit ${ }^{\circ}$ )

Die absoluten Werte im Säulendiagramm verdeutlichen die größte Achsabweichung bei der Whiteside-Linie und zeigen bei der bandspannungsbasierten Femurausrichtung den größten Unterschied - sowohl innerhalb der Rotationsausrichtungen als auch im Vergleich zu präoperativ (siehe Abb. 33).

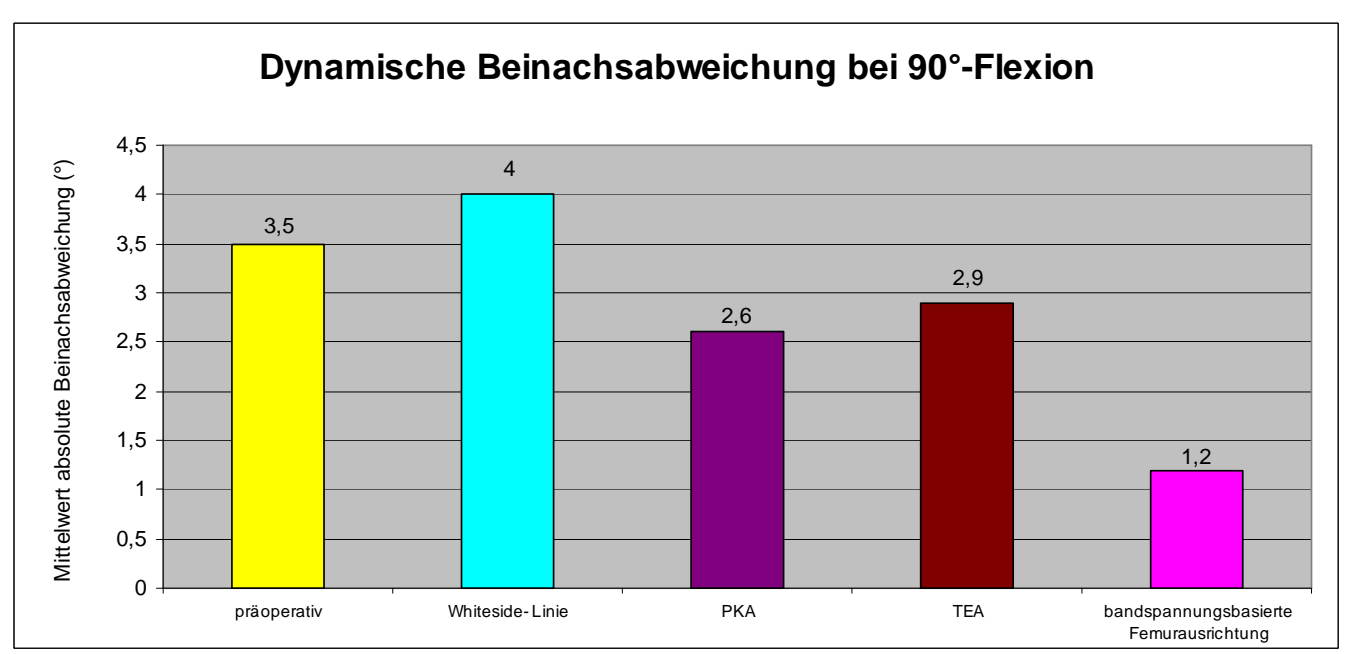

Abbildung 33: Vergleich absolute Abweichung dynamische Beinachse bei $90^{\circ}$-Flexion 
Bei der Gegenüberstellung der Mittelwerte in Streckstellung, d. h. bei einer ROM von $0^{\circ}$ sind die Mittelwerte nahezu identisch. Eine Verringerung der maximalen Varusabweichung von präoperativen $11,2^{\circ}$ auf maximal $2,7^{\circ}$ ist bei der TEA zu finden. Die anderen Rotationsausrichtungen weisen eine Abweichung von 3,3 $\mathrm{maxVar} / \mathrm{maxVal}$ auf. Bei einer minimalen Beinachsabweichung von $0,2^{\circ} \mathrm{minVar} / \mathrm{minVal}$ beträgt der Korridor 2,9 $9^{\circ}$. Im Boxplot sind nur bei der TEA keine Ausreißer vorhanden. Positive Werte kennzeichnen im Boxplot (Einheit "Grad") eine Varusabweichung, negative dementsprechend eine Valgusabweichung.

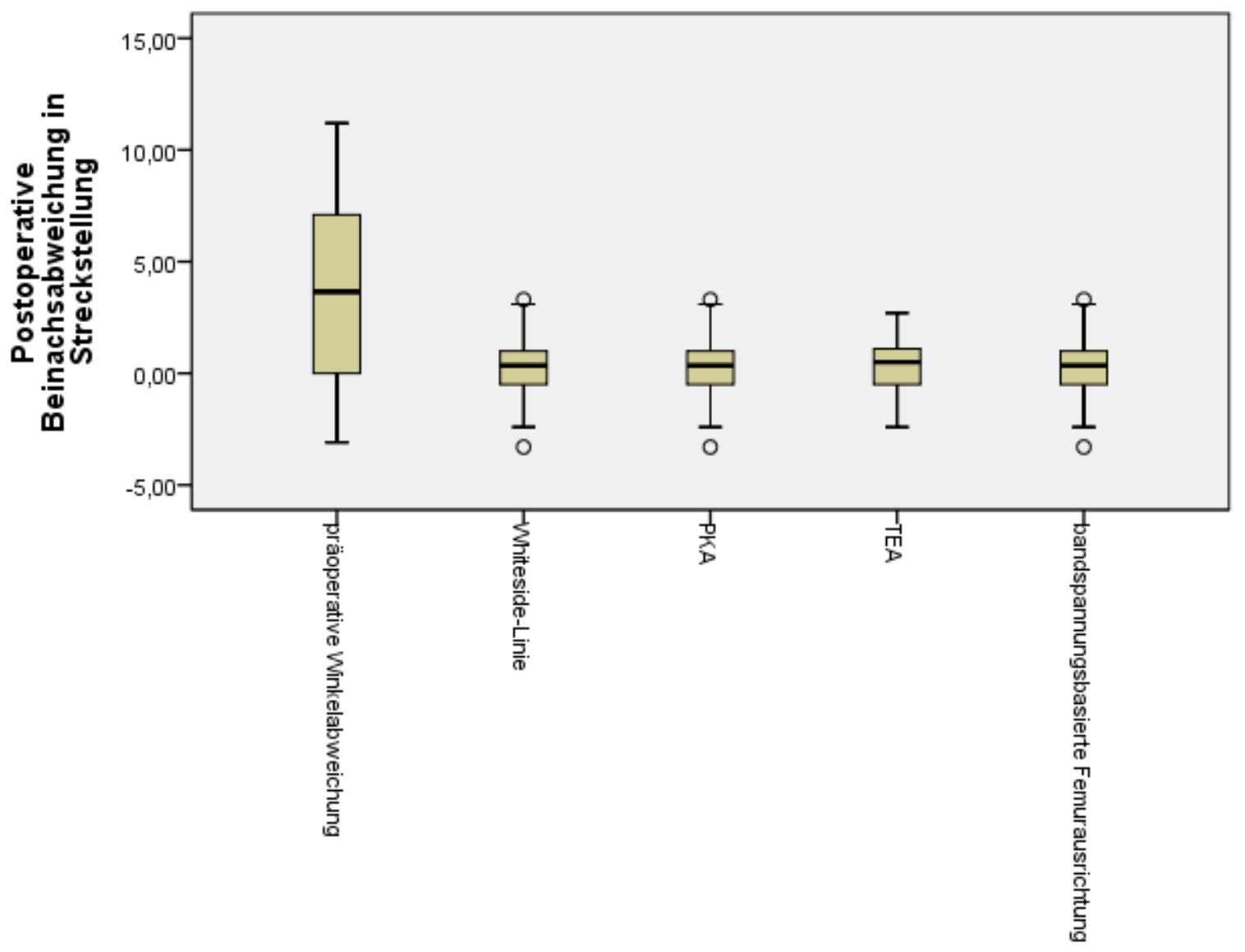

Abbildung 34: Boxplot mit Abweichung der Beinachse in Streckstellung (Einheit ${ }^{\circ}$ )

Die postoperative ROM-Auswertung in Streckstellung zeigt bei der Gegenüberstellung der Mittelwerte keine signifikanten Unterschiede zwischen den einzelnen Verfahren zur Bestimmung der femoralen Rotationsausrichtung. Aber es existiert mit $p<0.001$ ein signifikanter Unterschied von allen 4 Methoden im Vergleich zu präoperativ. 
Insgesamt ist bei der postoperativen ROM-Evaluation der mechanischen Beinachse bezogen auf die bandspannungsbasierte Femurausrichtung festzustellen, dass in $10 \%$ der Fälle $(n=9)$ eine Neutralstellung der Beinachse vorliegt (siehe Abb. 35). Bei $\mathrm{n}=58$ besteht postoperativ eine Varusabweichung bis maximal $3,3^{\circ}$. In Anbetracht der präoperativen Achsdeviation ist hier eine Zunahme der Valgusabweichung zu verzeichnen. Im Gegensatz zum präoperativen Streckdefizit bei $n=17$ tritt bei der dynamischen Auswertung keine Streckhemmung auf. Da es wie oben beschrieben keine signifikanten Unterschiede zwischen der Whiteside-Linie, TEA, PKA und der bandspannungsbasierten Femurausrichtung bei der postoperativen ROM-Evaluation in Streckstellung gibt, ist die prozentuale Verteilung repräsentativ.

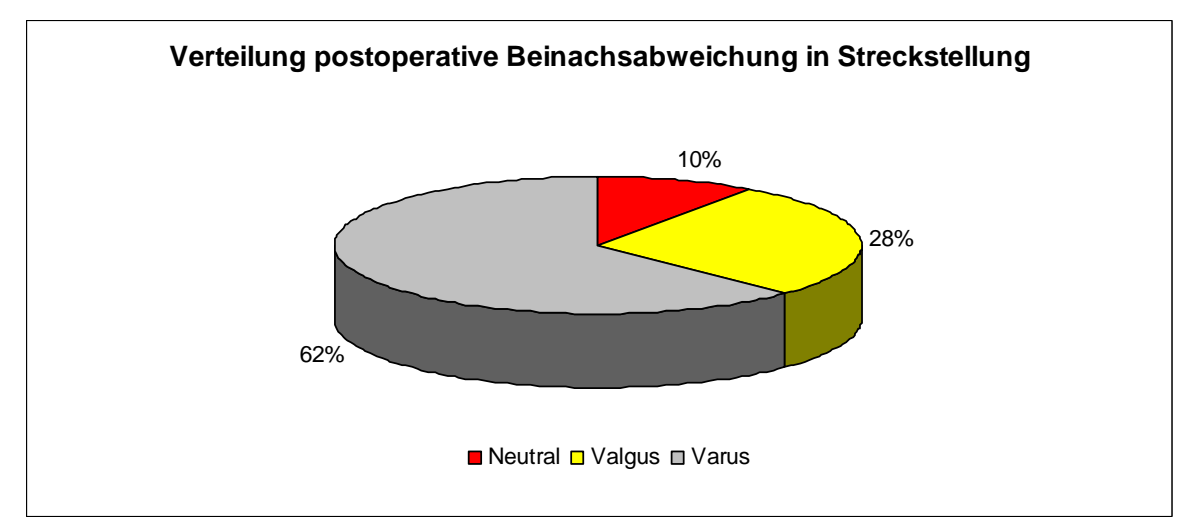

Abbildung 35: Diagramm postoperative Beinachsabweichung bei $0^{\circ}$ 


\subsection{Femorale Rotationsausrichtung bei Analyse des Beugespaltes} in Abhängigkeit der knöchernen Landmarken und mittels bandspannungsbasierter Navigation

Mit der Auswertung der 4 Verfahren zur Rotationsausrichtung der femoralen Implantatkomponente bezogen auf den Beugespalt erfolgt eine Überprüfung der Symmetrie. Die deskriptive Analyse der 3 knöchernen Landmarken untereinander ergibt die größten maximalen Rotationsabweichungen im Blick auf die WhitesideLinie $\left(\max I R O 12,2^{\circ} / \operatorname{maxARO} 15,5^{\circ}\right)$. Hier finden sich auch die größte Schwankungsbreite sowie der höchste Mittelwert der absoluten Winkelabweichung mit $3,9^{\circ}$ bei einer Standardabweichung von $3,13^{\circ}$. Die höchste zufällige Abweichung vom Mittelwert liegt demzufolge bei der Whiteside-Linie vor. Die Mittelwerte der absoluten Winkelabweichung von der TEA und der PKA sind annähernd gleich (siehe Tab. 5). Mit $p<0.01$ bei der PKA und $p<0.05$ bei der TEA besteht ein signifikanter Unterschied zur Whiteside-Linie.Die geringste Streuung ist bei der bandspannungsbasierten Femurausrichtung mit einer maximalen Innenrotation von $3,0^{\circ}$ und einer maximalen Außenrotation von $2,4^{\circ} \mathrm{zu}$ beobachten, gefolgt von der transepikondylären Achse (maxIRO 7,2\% maxARO 7,1 ${ }^{\circ}$. Mit dem engsten 95\%Konfidenzintervall von $\left[0,5^{\circ} ; 0,8^{\circ}\right]$ liefert die bandspannungsbasierte Femurausrichtung mit hoher Wahrscheinlichkeit im Mittel eine absolute Winkelabweichung von unter $1^{\circ}$ und hat zudem die geringste Streuung.

\begin{tabular}{|l|c|c|c|c|}
\hline & $\begin{array}{c}\text { Whiteside- } \\
\text { Linie }\end{array}$ & PKA & TEA & $\begin{array}{c}\text { bandspannungsbasierte } \\
\text { Femurausrichtung }\end{array}$ \\
\hline $\begin{array}{l}\text { Mittelwert absoluter } \\
\text { Winkelabweichung }\end{array}$ & $3,9^{\circ}$ & $2,8^{\circ}$ & $2,9^{\circ}$ & $0,6^{\circ}$ \\
\hline maxIRO & $12,2^{\circ}$ & $8,6^{\circ}$ & $7,2^{\circ}$ & $3,0^{\circ}$ \\
\hline maxARO & $15,5^{\circ}$ & $6,5^{\circ}$ & $7,1^{\circ}$ & $2,4^{\circ}$ \\
\hline SD & $3,13^{\circ}$ & $2,37^{\circ}$ & $2,23^{\circ}$ & $0,79^{\circ}$ \\
\hline CI & {$\left[3,3^{\circ} ; 4,6^{\circ}\right]$} & {$\left[2,3^{\circ} ; 3,3^{\circ}\right]$} & {$\left[2,2^{\circ} ; 3,6^{\circ}\right]$} & {$\left[0,5^{\circ} ; 0,8^{\circ}\right]$} \\
\hline
\end{tabular}

Tabelle 5: Femorale Rotationsabweichung zur tibialen Resektionsfläche bei $90^{\circ}$-Flexion 
Mit einem Mittelwert der absoluten Winkelabweichung von $0,6^{\circ}\left(\mathrm{SD}=0,79^{\circ}\right)$ (siehe Tab. 5) wird bei der bandspannungsbasierten Femurausrichtung mit $p<0.01$ eine signifikante Reduzierung der Winkelabweichung im Vergleich zu der Whiteside-Linie, der posterioren Kondylenachse und der transepikondylären Achse erreicht.

Die im Boxplot auf der y-Achse angegebenen Werte besitzen die Einheit "Grad“, wobei positive Werte eine femorale Innenrotation darstellen. Der Negativbereich zeigt die femorale Außenrotation an. Die größte Schwankungsbreite und der größte Interquartilsabstand unterliegen der Whiteside-Linie. Die bandspannungsbasierte Femurausrichtung präsentiert sich im Boxplot als die solideste Methode zur Rotationsausrichtung der Femurkomponente, da sie den kleinsten Abstand der Quartilen erzeugt (siehe Abb. 36). Ausreißer sind nur bei der Whiteside-Linie und der bandspannungsbasierten Femurausrichtung zu finden. Im letztgenannten Fall ist jedoch die geringste Spannweite festzustellen.

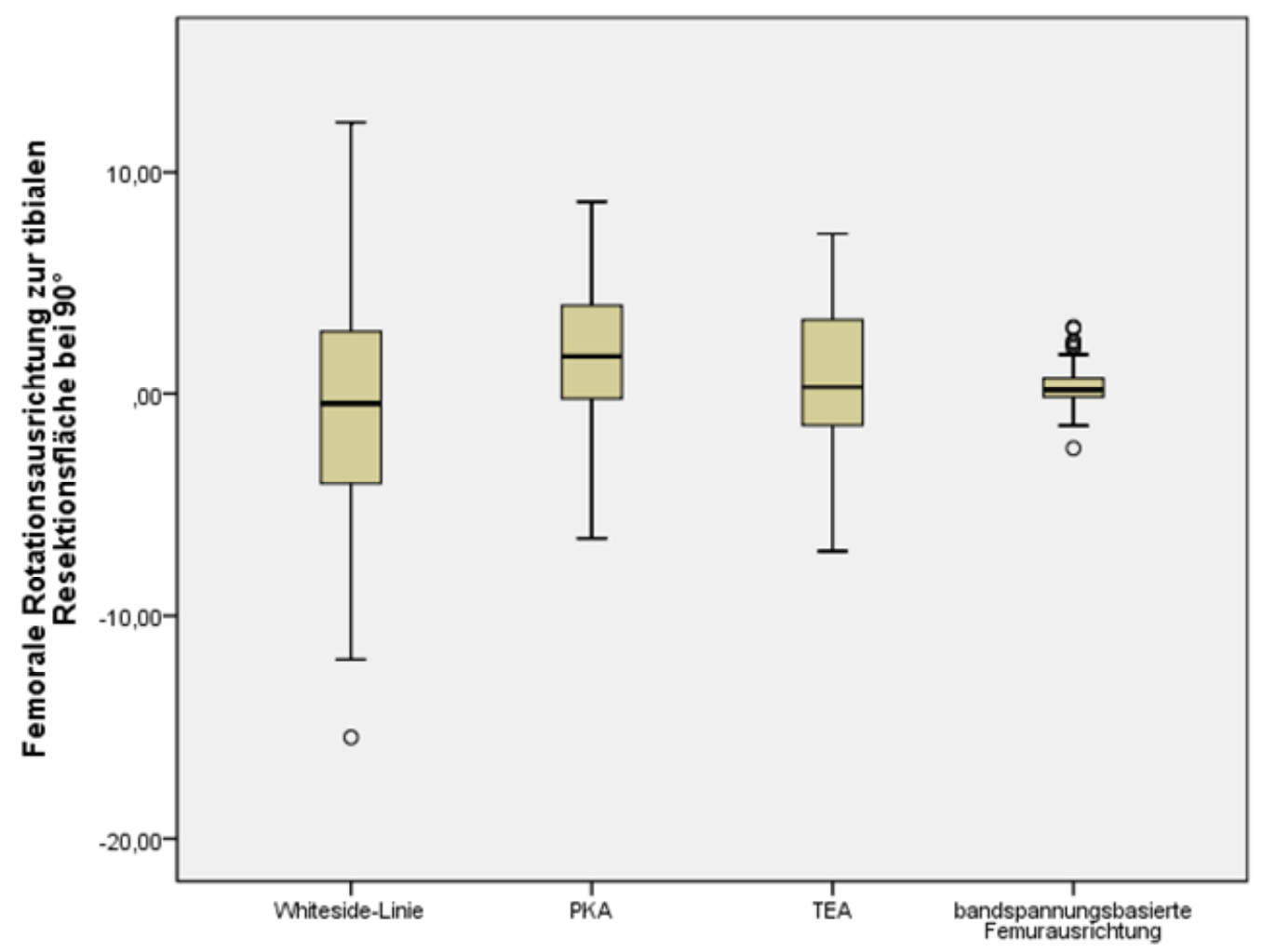

Abbildung 36: Boxplot zur Darstellung der Rotationsabweichung beim Beugespalt (Einheit ${ }^{\circ}$ ) 
Für eine Gegenüberstellung der 4 Methoden zur Rotationsausrichtung sind die Mittelwerte der absoluten Winkelabweichungen im folgenden Diagramm zusammengefasst. Hier wird der signifikante Unterschied der bandspannungsbasierten Femurausrichtung zu den 3 knöchernen Landmarken optisch verdeutlicht. Der höchste Mittelwert, d. h. die größte absolute Rotationsabweichung, besteht bei der Rotationsausrichtung mit der Whiteside-Linie.

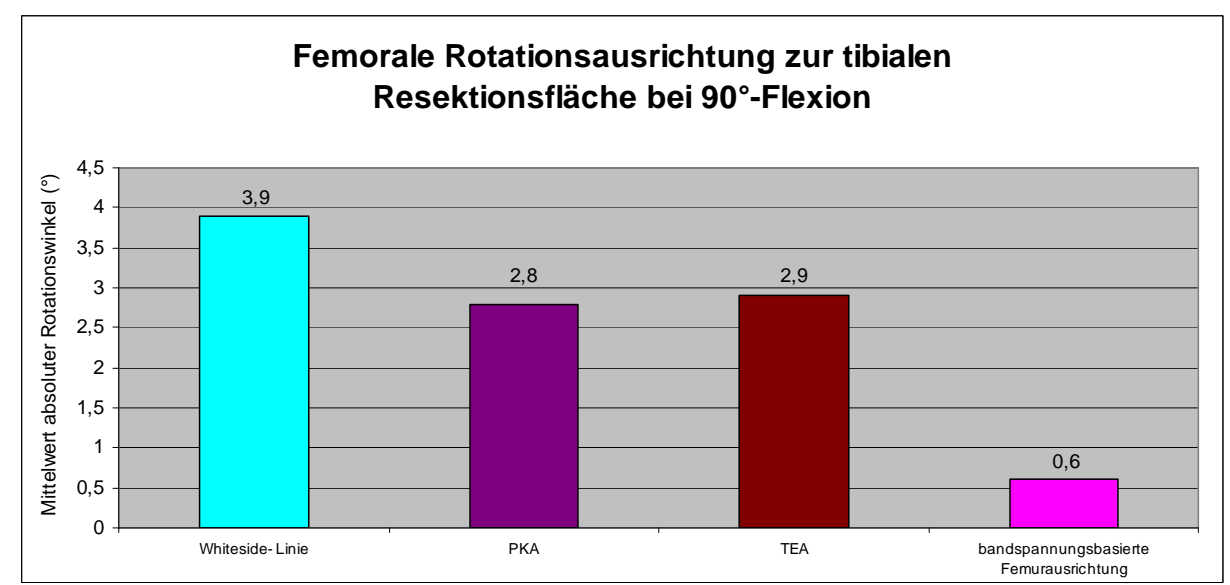

Abbildung 37: Vergleich Mittelwerte der absoluten Winkel der femoralen Rotationsausrichtungen zur tibialen Resektionsfläche beim Beugespalt 


\section{Diskussion}

Die Therapie von degenerativen Gelenkveränderungen nimmt bei einer stetig wachsenden Bevölkerungszahl und zunehmenden Lebenserwartung einen besonderen Stellenwert in der orthopädischen Behandlung ein.

Bei der radiologischen und klinischen Gonarthrose stellt zurzeit die Implantation einer Kniegelenkstotalendoprothese den "Goldstandard" dar. Die exakte Prothesenimplantation ist in der Kniegelenksendoprothetik hochrelevant, da die Implantate kaum Fehler verzeihen. Ergo ist die Genauigkeit der Implantationstechnik mit dem Ziel der Wiederherstellung der physiologischen Beinachsverhältnisse von entscheidender Bedeutung, um eine optimale Funktionalität und Stabilität sicher zu stellen (Hube et al. 2011). Die mechanisch korrekte Ausrichtung der Prothesenkomponenten, das so genannte alignement, ist Gegenstand zahlreicher Untersuchungen, da sich im Vergleich zur ständig wachsenden Anzahl von Erstimplantationen die Anzahl der Revisions-Operationen überproportional erhöht (Schnabel und Borelli 2011). Trotz des tribologischen Fortschritts der Prothesenkomponenten und hochentwickelter Operationsmethoden wird dadurch demonstriert, dass das Problem der limitierten Prothesenstandzeit bis dato nicht behoben ist (Schnabel und Borelli 2011). In verschiedenen Studien von zum Beispiel Bäthis et al. haben sich intraoperative Navigationssysteme als reliable Instrumente zur Verbesserung eines mechanischen alignements erwiesen (Bäthis et al. 2004; Chauhan et al. 2004; Bäthis et al. 2006; Bejek et al. 2007).

Die Fehlausrichtung der Implantatkomponenten und die Bandinstabilität sind u. a. verantwortlich für ein frühzeitiges Versagen einer Kniegelenkstotalendoprothese (Peterson et al. 1979; Townley 1985; Callaghan et al. 2004; Vessely et al. 2006; Claus und Scharf 2007; Graichen et al. 2007). Zudem führt eine fehlerhafte Rotationsausrichtung der Femurkomponente zu einer klinischen Problematik wie dem patellar maltracking und zu einer unbefriedigenden Beweglichkeit (Berger et al. 1998; Miller et al. 2001; Stöckl et al. 2004). Ein asymmetrischer Beugespalt mit konsekutiver unilateraler Instabilität sowie vermehrtem PE-Abrieb ist ebenfalls das Resultat eines femoralen Rotationsfehlers (Wasielewski et al. 1994; Kohn und Rupp 2000; Insall et al. 2002; Romero et al. 2007). 
Nach Sharkey et al. und Callaghan et al. besteht in Bezug auf die Anzahl der Revisionseingriffe eine Korrelation zur inkorrekten Rotationsausrichtung der Femurkomponente (Sharkey et al. 2002; Callaghan et al. 2004).

Grundsätzlich existieren 2 unterschiedliche OP-Techniken zur Rotationsausrichtung bzw. Bestimmung des alignements. Auf der einen Seite kann die femorale Implantakomponente knochenreferenziert anhand der 3 konventionellen knöchernen Landmarken in der femur first-Methode ausgerichtet werden, andererseits weichteilreferenziert oder, wie in der vorliegenden Studie angewendet, mit bandspannungsbasierter Navigation in tibia first-Technik. Bei der so genannten gap-Technik basiert die Ausrichtung der Femurkomponente auf dem Beugespalt in $90^{\circ}$-Flexion.

Zu den 3 knöchernen Landmarken zählen die Whiteside-Linie, die posteriore Kondylenachse sowie die transepikondyläre Achse. Jedoch ist keine dieser so genannten Hilfslinien zur einwandfreien Rotationsausrichtung unproblematisch, da aufgrund anatomischer Verhältnisse Messungenauigkeiten unvermeidbar sind (Kohn und Rupp 2000; Siston et al. 2005). In verschiedenen Kadaverstudien wurde untersucht wie sich die knöchernen Landmarken zueinander verhalten. Berger et al. kamen in ihrer Studie an 75 Kadaverbeinen zu dem Ergebnis, dass die transepikondyläre Achse als Hilfslinie zur Rotationsausrichtung der Femurkomponente aufgrund der hohen Variabilität nicht brauchbar sei (Berger et al. 1998). Im selben Jahr wurde eine Studie an 84 Patienten von Nagamine et al. zur Überprüfung bzw. Anwendbarkeit der Whiteside-Linie veröffentlicht. Darin zeigten die Autoren, dass die Whiteside-Linie weniger verlässlich ist als die transepikondyläre Achse (Nagamine et al. 1998). Davor wurden in der Kadaverstudie von Whiteside und Arima die TEA, PKA und die von innen beschriebene Whiteside-Linie untersucht. Sie publizierten einen Vorteil der Whiteside-Linie gegenüber der transepikondylären Achse beim X-Bein (Whiteside und Arima 1995). Die posteriore Kondylenachse spielt zufolge Whiteside und Arima bei der Valgusgonarthrose aufgrund der Entrundung der lateralen Femurkondyle eine untergeordnete Rolle, da sie einen Innenrotationsfehler provoziere. Poilvache et al. veröffentlichten 1996 ihre Studie zum Vergleich der Whiteside-Linie, der PKA und der TEA. 
Dabei fanden sie heraus, dass die Whiteside-Linie annähernd rechtwinklig zur transepikondylären Achse steht. Dies wiederum würde bedeuten, dass die Whiteside-Linie ebenso wie die TEA zur Rotationsausrichtung anwendbar ist, jedoch aufgrund anatomischer Verhältnisse besser zu bestimmen ist. Von Matsuda et al. wurde bei der Varusgonarthrose die posteriore Kondylenachse als die vertrauenswürdigste Hilfslinie beschrieben (Matsuda et al.1998). In der von Olcott und Scott veröffentlichten Studie aus dem Jahre 1999 zur Untersuchung der knöchernen Landmarken hinsichtlich eines symmetrischen Beugespaltes stellte sich die TEA in $90 \%$ als erfolgreich dar. Die Whiteside-Linie (83\%) und die PKA $(70 \%)$ zeigten sich diesbezüglich als eher ungeeignet.

In Anbetracht der genannten Studien herrscht folglich Uneinigkeit, welche der 3 knöchernen Landmarken bei der knochenreferenzierten femur first-Technik die verlässlichste Hilfslinie zur Rotationsausrichtung der femoralen Implantatkomponente darstellt.

Auch der Einsatz von Computernavigation konnte bisher keine verlässliche, reproduzierbare femorale Rotationsausrichtung bewirken (Siston et al. 2005). In der von Siston et al. durchgeführten Studie an Kadaverknien wurde gezeigt, dass im Vergleich zu einer navigiert bestimmten TEA die manuell erfassten 3 knöchernen Landmarken Abweichungen von bis zu $13^{\circ}$ Innenrotation und $16^{\circ}$ Außenrotation unter Referenzierung auf die digital registrierte TEA aufwiesen (Siston et al. 2005). Es bestand kein signifikanter Unterschied zwischen der Bestimmung der knöchernen Landmarken. Auch die computernavigierte Bestimmung der transepikondylären Achse erbrachte keine besseren Ergebnisse.

Bei Überprüfung der Hypothese, dass aus einer navigierten, bandspannungsbasierten Rotationsausrichtung der Femurkomponente ein funktionell besseres Ergebnis hinsichtlich der Beinachsabweichung und konsekutiv der Flexionsstabilität resultiert, wurde ein simultaner (virtueller) Vergleich unter kinematischen Bedingungen und bei $90^{\circ}$-Flexion mit den 3 knöchernen Landmarken in der vorliegenden Studie angestellt. 
Die gleichzeitig, d.h. an demselben Patienten, durchgeführte, dynamische Gegenüberstellung von der Whiteside-Linie, der PKA sowie der TEA und der angewendeten bandspannungsbasierten Femurausrichtung unter Navigation stellt die Innovation dieser Studie dar. Ein solcher Vergleich anhand der intraoperativen Messungen wurde bisher in der Literatur noch nicht beschrieben. Mit den ROMMessungen wurde ein synchroner Vergleich der Kniegelenksfunktion bzgl. der Extension und Flexion bei gleichzeitiger Darstellung der Varus- bzw. Valgusabweichung in Abhängigkeit der 4 beschriebenen Methoden zur femoralen Rotationsausrichtung an einem Patienten möglich. Im Rahmen der vorliegenden Studie steht durch Berechnung der jeweiligen Winkelabweichungen aus den Funktionsdiagrammen eine Maßzahl zur Verfügung, mit der sich das Ausmaß der Varus- und Valgusabweichung im gesamten Bewegungsausmaß quantifizieren und somit vergleichbar machen lässt. Die Analyse der Funktionsdiagramme konnte mit $\mathrm{p}<0.001$ eine statistisch signifikante Reduktion der Winkelabweichungen unter Anwendung der 3 knöchernen Landmarken und der bandspannungsbasierten Femurausrichtung im Vergleich zu präoperativ aufzeigen. Somit konnte mit jedem Verfahren eine Verbesserung der präoperativen Gelenkfunktion bezüglich einer verminderten Varus-/Valgusabweichung erzielt werden. Des Weiteren zeigte sich bei der Auswertung der Funktionsdiagramme mit einem Mittelwert von $3,1^{\circ}$ die größte postoperative Winkelabweichung von der Ideallinie $\left(0^{\circ}\right.$-Achse) bei der WhitesideLinie. Ebenso wies die Whiteside-Linie hier die größte Streuung auf $\left(S D=2,09^{\circ}\right)$. Die Mittelwerte der TEA ( $\min .0,4^{\circ} / \max .6,5^{\circ}$ ) und der PKA (min. 0,2\% max. 7,5 $)$ lagen nahe beieinander, so dass bei funktioneller Betrachtung kein wesentlicher Unterschied zwischen diesen beiden Methoden zu erwarten ist. Der kleinste Mittelwert mit $1,1^{\circ}\left(\min .0,2^{\circ} / \max .2,8^{\circ}\right)$ und die solideste Streuung war bei der bandspannungsbasierten Femurausrichtung zu finden $\left(S D=0,58^{\circ}\right)$. Mit dieser Methode konnte ein signifikanter Unterschied $(p<0.01)$ zu den 3 konventionellen knöchernen Landmarken gezeigt werden. Verglichen mit der Studie von Matziolis et al. hat sich die Orientierung an der transepikondylären Achse als zuverlässiger als die gap-Technik herausgestellt, da sie annähernd parallel zu der ursprünglichen Flexionsachse des Kniegelenks ist (Matziolis et al. 2011). Aufgrund der in der Literatur beschriebenen, fehlenden klinischen Relevanz im Vergleich zur femur firstTechnik ist es kritisch zu betrachten, demzufolge bei der gap-Technik von einer Achsabweichung zu sprechen (Matziolis 2011). 
Singh et al. hingegen sprechen von besseren klinischen Ergebnissen unter Anwendung der gap-Technik (Singh et al. 2012). Eine große Streuung der Messdaten in Bezug auf die Whiteside-Linie wurde bereits in verschiedenen Studien belegt (Akagi et al. 2001; Middleton et al. 2007).

Da ein femoraler Rotationsfehler eine verstärkte einseitige Scherbelastung am PEInlay sowie eine unilaterale Instabilität hervorrufen kann, war die Analyse bei $90^{\circ}$ Flexion ein wesentlicher Bestandteil dieser Studie.

Die ROM-Evaluation bei $90^{\circ}$-Flexion demaskierte bei der Whiteside-Linie sogar eine Zunahme der maximalen Varusabweichung auf $12,8^{\circ}$ im Vergleich zu präoperativ (maxVar 10,4 ${ }^{\circ}$. Auch war der Mittelwert der absoluten Abweichung mit 4, $0^{\circ}$ um $0,5^{\circ}$ zum präoperativen Wert erhöht. Die größte Standardabweichung und das breiteste 95\%-Konfidenzintervall entstanden bei der Rotationsausrichtung entsprechend der Whiteside-Linie. Diese zeigte ebenso wie die TEA mit $p>0.05$ keine signifikante postoperative Verbesserung der Achsdeviation bei $90^{\circ}$-Flexion. Hier wäre folglich mit einer möglichen einseitigen tibio-femoralen Überbelastung und einer Bandinstabilität mit Auswirkungen auf die Funktion der Kniegelenksprothese zu rechnen. Mit der

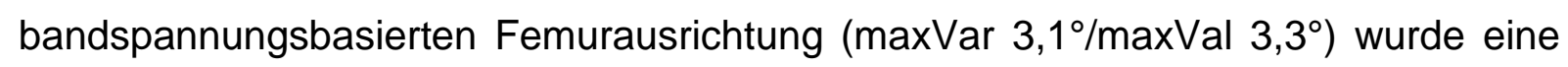
absolute Abweichung von $1,2^{\circ}$ erreicht. Dies deckt sich annähernd mit der von Bäthis et al. im Jahr 2006 veröffentlichten Studie, nach der ein postoperativer Korridor von $3^{\circ}$ Varus/Valgus durch die computerassistierte Kniegelenksprothesenimplantation im Vergleich zur konventionellen bzw. manuellen OP-Technik hoch signifikant ist (Bäthis et al. 2006). Mit $p<0.001$ wurde eine signifikante Verbesserung der Varus-/ Valgusabweichung durch die bandspannungsbasierte Femurausrichtung erzielt. Zugleich konnte die PKA $(p<0.05)$ eine signifikante Korrektur der Achsabweichung erwirken.

Die Auswertung der Rotationsabweichungen der 4 Verfahren (Whiteside-Linie, PKA, TEA und bandspannungsbasierte Femurausrichtung) von der tibialen Resektionsfläche bei $90^{\circ}$-Flexion ergab eine große Streuung innerhalb der maximalen Abweichungen. Die Whiteside-Linie stellte sich auch hierbei als die fehlerhafteste Hilfslinie (maxIRO $12,2^{\circ} / \operatorname{maxARO} 15,5^{\circ}$ ) heraus. Sie wies mit einem Mittelwert von $3,9^{\circ}$ bei der absoluten Winkelabweichung den größten Rotationsfehler auf. 
Danach folgte die TEA $\left(\operatorname{maxIRO} 7,2^{\circ} / \operatorname{maxARO} 7,1^{\circ}\right.$ ) mit einem Mittelwert von 2,9 . Die PKA zeigte zwar mit 2,8 einen kleineren Mittelwert im Vergleich zu der TEA, präsentierte aber auch den zweithöchsten maximalen Innenrotationsfehler mit 8,6 $6^{\circ}$. Mit der bandspannungsbasierten Femurausrichtung konnte bei einem Mittelwert von $0,6^{\circ}$ (maxIRO $3,0^{\circ} / \operatorname{maxARO} 2,4^{\circ}$ ) eine signifikant verbesserte Rotationsausrichtung der femoralen Implantatkomponente im Vergleich zu den 3 knöchernen Landmarken hergestellt werden $(p<0.01)$.

Hofmann et al. kamen in ihrer 2003 veröffentlichten Studie zu dem Ergebnis, dass Rotationsabweichungen von $>3^{\circ}$ bereits Auswirkungen mit mittlerer Schwere auf das klinische Resultat haben (Hofmann et al. 2003). Dabei wird allgemein angenommen, dass ein Innenrotationsfehler eher klinische Probleme auslöst als ein Außenrotationsfehler der Femurkomponente. Mit dem Verfahren der bandspannungsbasierten Femurausrichtung lagen alle maximalen Rotationswerte innerhalb eines Korridors von $3^{\circ}$. Somit stellt das veränderte operative Vorgehen eine verlässliche Methode zur Rotationsausrichtung der Femurkomponente für den klinischen Alltag dar. Analog zu der von Classen et al. 2011 publizierten Studie, in der die tibia first-Technik ein probates Verfahren zur Erzielung eines symmetrischen Beugespalts darstellt, konnte mit der bandspannungsbasierten Femurausrichtung ein weitestgehend paralleler Beugespalt mit Abweichungen in einem akzeptablen Korridor erreicht werden. Daher kann mit Blick auf die Standardweichung $\left(S D=0,79^{\circ}\right)$ und das $95 \%$-Konfidenzintervall von $\left[0,5^{\circ} ; 0,8^{\circ}\right]$ postuliert werden, dass unter Anwendung der bandspannungsbasierten femoralen Rotationsausrichtung eine optimierte funktionelle Rotation erreicht wird.

Die Analyse der ROM-Messungen in Streckstellungen hat bei $87 \%$ der Patienten eine präoperative Varusfehlstellung (maxVar $11,2^{\circ}$ ) sowie folglich bei $13 \%$ eine Valgusachsdeviation ( $\operatorname{maxVal} 3,1^{\circ}$ ) ergeben. Das Streckdefizit bei $n=17$ wurde in allen Fällen ausgeglichen. Postoperativ wurde bei $10 \%$ der Fälle mit der Neutralstellung eine optimale Beinachse erzielt. Alle Werte lagen innerhalb eines Korridors von $3,3^{\circ}$ Varus-/Valgusabweichung. Eine Zunahme der postoperativen Valgusabweichung in diesen Grenzen war postoperativ mit 28\% zu verzeichnen. 
In Zusammenschau aller Ergebnisse bzw. Mittelwerte der Auswertungen hat sich die Rotationsausrichtung der femoralen Implantatkomponente mit bandspannungsbasierter Navigation als reliabel und korrekt herausgestellt. Verglichen mit einer im Jahr 2008 veröffentlichten Studie von Yau et al. verursachte hier die bandspannungsbasierte navigierte Femurausrichtung analog zur gap-Technik die geringsten Malrotationen (Yau et al. 2008). In einer Publikation von Bäthis et al. wurde 2006 anhand einer Metaanalyse untersucht, ob die Computernavigation bei der Kniegelenksprothesenimplantation einen Vorteil gegenüber der konventionellen bzw. manuellen OP-Technik besitzt. Hier wurde kein Vorteil von Navigationssystemen gesehen. Lüring et al. hingegen berichten über einen Vorteil der Computernavigation beim ligament balancing (Lüring et al. 2006). Kritisch wird u. a. von Katz et al. die intraoperative Bestimmung der knöchernen Landmarken gesehen, da die manuelle Erfassung abhängig ist vom Operateur und zu enormen Abweichungen führt (Katz et al. 2001).

Die Schwäche der vorliegenden Studie liegt in den nicht unter Varus- bzw. Valgusstress oder unter axialer Last durchgeführten Funktionsdiagrammen. Des Weiteren wurden nur Fälle aufgenommen, bei denen die präoperative mechanische Beinachse eine maximale Varus-/Valgusabweichung von $15^{\circ}$ in der a.p.-Ganzbeinstandaufnahme aufwies. Des Weiteren muss die allgemeine bisherige Unkenntnis hinsichtlich der präoperativen femoralen Rotation bzw. der physiologischen Rotation als ein generelles Problem in Studien dieser Art angesehen werden. Eine mögliche Fehlrotation in Bezug zur präexistenten, unbekannten pyhsiologischen Femurrotation wird bei der Studiennavigation in Kauf genommen, wenn dafür ein balancierter Beugespalt erreicht wird. Aufgrund der Prothesendesigns sind stabile, ausgeglichene Bandverhältnisse zu bevorzugen, da sie für die Funktionalität einer Kniegelenkstotalendoprothese von Relevanz sind. Intraoperativ wurden die Bandverhältnisse nach Abtragung der Osteophyten daraufhin überprüft, ob die Beinachse aufgrund von Bandkontrakturen nicht korrigierbar ist. Eine Weichteilbalancierung fand nur durch das zugangsbedingte erstgradige mediale release statt. Letztlich wurde bei der veränderten OP-Technik ein paralleler, balancierter Beugespalt durch eine knöcherne Resektion geschaffen. Eine weitere Schwäche der Studie stellt die fehlende Kontrolle des Beugespaltes nach durchgeführter dorsaler Femurresektion dar. 
Die bandspannungsbasierte OP-Technik unterliegt zudem verschiedenen Limitierungen. Präoperativ ausgeprägte Beinachsdeviationen mit konsekutiven Kontrakturen können vermutlich mit der bandspannungsbasierten Femurausrichtung nicht korrigiert werden, weswegen als Einschlusskriterium die maximale Varus-/ Valgusabweichung von $15^{\circ}$ definiert wurde und intraoperativ die Bandverhältnisse auf ihre Intaktheit überprüft wurden. Die angewendete navigierbare Femurgrößenlehre lässt eine Korrektur des Beugespaltes hinsichtlich der Rotation bis maximal $+/-6^{\circ} \mathrm{zu}$. Eine knöcherne Korrektur von mehr als $+/-6^{\circ}$ zur Erzielung eines parallelen balancierten Beugespaltes ist hiermit nicht möglich.

Insgesamt lässt sich anhand der Studienergebnisse schlussfolgern, dass die bandspannungsbasierte gap-Technik in Kombination mit einem Navigationssystem im Gegensatz zu den 3 knöchernen Landmarken ein vertrauenswürdiges Verfahren zur Rotationsausrichtung der Femurkomponente bei der Kniegelenksprothesenimplantation darstellt, da die gemessenen Achsdeviationen der bandspannungsbasierten, navigierten Femursausrichtung im Funktionsdiagramm am ehesten auf die Ungenauigkeit der tatsächlichen knöchernen Resektion zurückzuführen sind. Einseitige Überbelastungen und unilaterale Instabilitäten können mit der veränderten OP-Technik vermieden und der PE-Abrieb kann infolge gleichmäßiger mechanischer Belastung minimiert werden.

Somit kann mit der bandspannungsbasierten Femurausrichtung entsprechend der aufgestellten Hypothese 1 eine funktionell korrekte Positionierung erreicht werden. Die Hypothese 2 konnte durch Anwendung der Studiennavigation und konsekutiver Datenverarbeitung mit dem „Gena Light Interpreter" bewiesen werden.

Die Evaluation der klinischen Ergebnisse unter Anwendung der bandspannungsbasierten, navigierten Rotationsausrichtung der Femurkomponente ist Gegenstand einer weiteren, derzeit laufenden Untersuchung. Eine Korrelation der klinischen Ergebnisse in Abhängigkeit mit den unterschiedlichen Techniken zur femoralen Rotationsausrichtung ist mit diesem Studiendesign nicht untersuchbar. Hier wäre die Durchführung einer matched-pairs-Studie notwendig. 


\section{Zusammenfassung}

Derzeit stellt die Primärimplantation einer Kniegelenkstotalendoprothese im klinischen Alltag ein Standardverfahren in der operativen Behandlung der Gonarthrose dar und ist eine der häufigsten endoprothetischen Operationen in Deutschland. Für den operativen Behandlungserfolg ist die intraoperativ korrekte Ausrichtung der femoralen Implantatkomponente von Bedeutung. Trotz 3 etablierter knöcherner Landmarken zur Bestimmung der femoralen Rotation hat sich keine der genannten Hilfslinien als unproblematisch herausgestellt. Daher wurde ein neues Operationsverfahren - die „bandspannungsbasierte, navigierte Rotationsausrichtung der femoralen Prothesenkomponente" - mit dem Ziel der optimierten, funktionellen femoralen Rotation zur Verbesserung der Kniegelenksstabilität und Vermeidung eines vermehrten PE-Verschleißes zur Anwendung gebracht.

Im Rahmen der vorliegenden Studie wurde 93 konsekutiven Patienten mit primärer Gonarthrose und entsprechendem Leidensdruck in der beschriebenen navigierten Operationstechnik eine Kniegelenkstotalendoprothese Typ EFK ${ }^{\circledR}$ der Firma Smith \& Nephew primär implantiert. Anhand der intraoperativ registrierten konventionellen knöchernen Landmarken sowie der bandspannungsbasierten Rotation der Femurkomponente durch das Navigationssystem PiGalileo TKR Light ${ }^{\circledR}$, Version 1.3 der Smith \& Nephew GmbH konnte mit Hilfe des Auswertungsprogramms „Gena Light Interpreter" (AXIOS 3D ${ }^{\circledR}$ Services $\mathrm{GmbH}$ ) ein simultaner Vergleich der jeweiligen Ausrichtungen bzgl. der Femurrotation untereinander an dem zu operierenden Kniegelenk graphisch und mathematisch dargestellt werden.

Aus den Ergebnissen dieser Studie lässt sich schlussfolgern, dass sich die knöchernen Landmarken zur femoralen Rotationsausrichtung als nicht zuverlässig und ungenau herausgestellt haben. In der vorliegenden Arbeit konnte in allen Fällen ein balancierter, weitestgehend paralleler Beugespalt unter Anwendung der Femurausrichtung mit bandspannungsbasierter Navigation erreicht werden. Hier zeigte sich bei den durchschnittlichen absoluten Werten ein Rotationsfehler von $0,6^{\circ}$ (maxIRO 3,0\%/maxARO 2,4\%). 
Mit 2,8 $8^{\circ}$ bei der posterioren Kondylenachse (maxIRO 8,6\% maxARO $6,5^{\circ}$ ) und 2,9 bei der transepikondylären Achse $\left(\max I R O \quad 7,2^{\circ} / \operatorname{maxARO} 7,1^{\circ}\right.$ ) lagen die Mittelwerte der absoluten Winkelabweichung dieser 2 knöchernen Landmarken zwar eng beieinander, doch der Abstand der Durchschnittswerte zur bandspannungsbasierten Femurausrichtung betrug mehr als das 3,5-fache. Die Whiteside-Linie, welche im klinischen Alltag oft zur Anwendung kommt, hat sich mit einem durchschnittlichen absoluten Wert von $3,9^{\circ}$ bei einem maximalen Innenrotationsfehler von $12,2^{\circ}$ sowie einem maximalen Außenrotationsfehler von $15,5^{\circ}$ als die ungenaueste Hilfslinie zur Rotationsausrichtung der Femurkomponente herausgestellt. Insgesamt wurde mit der bandspannungsbasierten Femurausrichtung eine statistisch signifikante Verbesserung der femoralen Rotationausrichtung erzielt.

Die klinischen Resultate der Rotationsausrichtung der femoralen Implantatkomponente mit bandspannungsbasierter Navigation stehen, auch im Vergleich zu den knöchernen Landmarken, noch zur Diskussion.

Zum ersten Mal wurden die digitalisiert erfassten 3 konventionellen knöchernen Landmarken als messbare Kenngrößen virtuell mit der in der Arbeit favorisierten bandspannungsbasierten, navigierten Rotationsausrichtung der Femurkomponente an einem Patienten bzw. Kniegelenk synchron untersucht und deren Einfluss auf die Kniegelenksfunktion in einem Funktionsdiagramm berechnet.

Eine optimierte funktionelle femorale Rotationausrichtung konnte mit der navigierten Studien-Operationstechnik aufgezeigt werden. Eine unilaterale Instabilität sowie eine einseitige Überlastung des PE-Inlays kann so bei guter Funktionalität möglicherweise vermieden werden, wodurch die Prothesenstandzeit entsprechend wahrscheinlich verlängert werden kann. Dies ist Gegenstand noch weiterer Untersuchungen. 


\section{Literaturverzeichnis}

Akagi M, Yamashita E, Nakagawa T, Asano T und Nakamura T (2001): Relationship between frontal knee alignment and reference axes in the distal femur. Clin Orthop Relat Res $\underline{388}, 147-56$

Andersen HN und Dyhre-Poulsen P (1997): The anterior cruciate ligament does play a role in controlling axial rotation in the knee. Knee Surg Sports Traumatol Arthrosc $\underline{5}, 145-9$

Bäthis H, Perlick L, Tingart M, Lüring C, Zurakowski D und Grifka J (2004): Alignment in total knee arthroplasty. A comparison of computer-assisted surgery with the conventional technique. J Bone Joint Surg $\mathrm{Br} \underline{86}, 682-7$

Bäthis H, Perlick L, Tingart M, Perlick C, Lüring C und Grifka J (2005):

Intraoperative cutting errors in total knee arthroplasty. Arch Orthop Trauma Surg 125, 16-20

Bäthis H, Shafizadeh S, Paffrath T, Simanski C, Grifka J und Lüring C (2006): Sind navigierte Knieendoprothesen tatsächlich präziser implantiert? Eine Metaanalyse vergleichender Studien. Orthopäde 35, 1056-65

Bejek Z, Solyom L und Szendroi M (2007): Experiences with computer navigated total knee arthroplasty. Int Orthop $\underline{31}, 617-22$

Benninghoff A: Anatomie. Makroskopische Anatomie, Embryologie und Histologie des Menschen, Band 1. 15. Auflage; Urban \& Schwarzenberg, München 1994 Berger RA, Rubash HE, Seel MJ, Thompson WH und Crossett LS (1993): Determining the rotational alignment of the femoral component in total knee arthroplasty using the epicondylar axis. Clin Orthop Relat Res 286, 40-7 Berger RA, Crossett LS, Jacobs JJ und Rubash HE (1998): Malrotation causing patellofemoral complications after total knee arthroplasty. Clin Orthop Relat Res $\underline{356}$, 144-53

Bernau A: Orthopädische Röntgendiagnostik. 3. Auflage; Urban \& Schwarzenberg, München 1995

Bertsch C, Holz U, Konrad G, Vakili A und Oberst M (2007): Klinische und radiologische Ergebnisse nach navigierter Knieendoprothetik: Analyse einer kontrollierten und prospektiven Studie im Vergleich zur konventionellen Implantationstechnik. Orthopäde $\underline{36}, 739-45$ 
BQS (2007): BQS-Bundesauswertung 2006. Knie-Totalendoprothesen-

Erstimplantation. Online verfügbar unter:

http://www.bqs-outcome.de/2006/ergebnisse/leistungsbereiche/knie_tep_erst/ datengrundlage [Recherchedatum: 17.08.2007]

Briard JL, Witoolkollachit P, Lin G (2007): Weichteilmanagement in der Knieendoprothetik. Untersuchung der Ligamentbalancierung. Orthopäde $\underline{36}$, 635-642

Büttner-Janz K und Ekkernkamp A (2002): Einflussfaktoren auf die Patellaführung in der Knieendoprothetik und operatives Weichteilmanagement. Z Orthop Ihre Grenzgeb $\underline{140}$, 27-31

Callaghan JJ, O'Rourke MR und Saleh KJ (2004): Why knees fail: lessons learned. J Arthroplasty $\underline{19}, 31-4$

Chauhan SK, Scott RG, Breidahl W und Beaver RJ (2004): Computer-assisted knee arthroplasty versus a conventional jig-based technique. A randomised, prospective trial. J Bone Joint Surg Br $\underline{\text { 86, 372-7 }}$

Cicuttini F, Wluka A, Hankin J, Wang Y (2004): Longitudinal study of the relationship between knee angle and tibiofemoral cartilage volume in subject with knee osteoarthritis. Rheumatology $\underline{43}, 321-4$

Classen T, Wegner A, Müller RD und von Knoch M (2011): Accuracy of rotational positioning of the femoral component using the tibial-cut-first technique. Z Orthop Unfall 149(6), 626-9

Claus A und Scharf HP (2007): "Ligament balancing" bei Varusgonarthrose. Orthopäde 36, 643-4, 646-9

Debrunner AM: Orthopädie/Orthopädische Chirurgie. 4. Auflage; Verlag Hans Huber, Bern 2005

DGOOC: Leitlinien Orthopädie. 2. Auflage; Deutscher Ärzte-Verlag, Köln 2002 DIMDI (2012): Kapitel XIII. Krankheiten des Muskel-Skelett-Systems und des Bindegewebes (M00-M99). Online verfügbar unter:

http://www.dimdi.de/static/de/klassi/diagnosen/icd10/htmlamt/fr-icd.htm?gm15.htm+ [Recherchedatum: 10.05.2012]

Fehring TK (2000): Rotational malalignment of the femoral component in total knee arthroplasty. Clin Orthop Relat Res $\underline{380}, 72-9$

Fehring TK und Valadie AL (1994): Knee instability after total knee arthroplasty. Clin Orthop Relat Res 299, 157-62 
Graichen H, Strauch M, Katzhammer T, Zichner L und von Eisenhart-Rothe R (2007): Ligamentäre Instabilität bei Knie-TEP - Ursachenanalyse. Orthopäde $\underline{36}$, 650, 652-6

Heck DA, Robinson RL, Partridge CM, Lubitz RM und Freund DA (1998): Patient outcomes after knee replacement. Clin Orthop Relat Res $\underline{356}$, 93-110 Hofmann S, Romero J, Roth-Schiffl E und Albrecht T (2003):

Rotationsfehlstellungen der Komponenten als Ursache chronischer Schmerzen und vorzeitigem Prothesenversagen bei Knieendoprothesen. Orthopäde 32, 469-76 Hollis JM, Takai S, Adams DJ, Horibe S und Woo SL (1991): The effects of knee motion and external loading on the length of the anterior cruciate ligament (ACL): a kinematic study. J Biomech Eng $\underline{113}$, 208-14

Hube R, Mayr HO, Kalteis T und Matziolis G (2011): Extension first technique for TKA implantation. Oper Orthop Traumatol $\underline{23}, 241-8$

Insall JN, Scuderi GR, Komistek RD, Math K, Dennis DA und Anderson DT (2002): Correlation between condylar lift-off and femoral component alignment. Clin Orthop Relat Res $\underline{403}$, 143-52

Janssen J und Laatz W: Statistische Datenanalyse mit SPSS für Windows.

6. Auflage; Springer Verlag, Berlin 2007

Jerosch J und Floren M (2000): Lebensqualitätsgewinn (SF-36) nach Implantation einer Knieendoprothese. Unfallchirurg 103, 371-4

Jerosch J, Heisel J und Imhoff AB: Fortbildung Orthopädie und Traumatologie 12. Knie. Steinkopff Verlag, Darmstadt 2007

Kalairajah Y, Cossey AJ, Verrall GM, Ludbrook G und Spriggins AJ (2006): Are systemic emboli reduced in computer-assisted knee surgery? A prospective, randomised, clinical trial. J Bone Joint Surg Br $\underline{88}, 198-202$

Katz MA, Beck TD, Silber JS, Seldes RM und Lotke PA (2001): Determining femoral rotational alignment in total knee arthroplasty: reliability of techniques. J Arthroplasty $\underline{16}$ (3), 301-5

Kellgren JH und Lawrence JS (1957): Radiological assessment of osteo-arthrosis. Ann Rheum Dis 16, 494-501

Kohn D und Rupp S (1999): Alloarthroplastik des Kniegelenkes. Orthopäde $\underline{28}$, 97595

Kohn D und Rupp S (2000): Knieendoprothetik - Operationstechnische Aspekte.

Orthopäde 29, 697-707 
König A, Walther M, Kirschner S und Gohlke F (2000): Balance sheets of knee and functional scores 5 years after total knee arthroplasty for osteoarthritis: a source for patient information. J Arthroplasty 15, 289-94

Koschack J (2008): Standardabweichung und Standardfehler: der kleine, aber feine Unterschied. Z Allg Med 2008 84, 258-60

Kowalski M und Gorecki A (2004): Total knee arthroplasty using the OrthoPilot computer-assisted surgical navigation system. Ortop Traumatol Rehabil $\underline{6}$, 456-60 Kubein-Meesenburg D, Nägerl H und Fanghänel J (1991): Elements of a general theory of joints. 5. Basic mechanics of the knee. Anat Anz 173, 131-42

Kubein-Meesenburg D, Nägerl H, Cotta H und Fanghänel J (1993):

Biomechanische Prinzipien in Diarthrosen und Synarthrosen. Teil I: Grundbegriffe bei Diarthrosen. Z Orthop Ihre Grenzgeb 131, 97-104

Kwiatkowski K (2004): Gonarthrosis-epidemiology and risk factors. Pol Merkur Lekarski 17, 410-4

Luo CF (2004): Reference axes for reconstruction of the knee. Knee 11, 251-7

Lüring C, Perlick L, Tingart M, Bäthis H und Grifka J (2006): Fortschritte im Weichteilmanagement in der Knieendoprothetik. Einsatz von bildfreien Navigationssystemen. Orthopäde $\underline{35}$ (10), 1066-72

Lüring C, Perlick L, Bäthis H, Tingart M und Grifka J (2007): The effect of femoral component rotation on patellar tracking in total knee arthroplasty. Orthopedics $\underline{30}$, 965-7

Matsuda S, Matsuda H, Tomoyuki M, Sasaki K, Iwamoto Y, Miura H (1998):

Femoral Condyle Geometry in Normal and Varus Knee. Clin Orthop Relat Res $\underline{349}$, 183-8

Matziolis G, Boenicke H, Pfiel S, Wassilew G und Perka C (2011): The gap technique does not rotate the femur parallel to the epicondylar axis. Arch Orthop Trauma Surg 131(2), 163-6

Middleton FR und Palmer SH (2007): How accurate is Whiteside's line as a reference axis in total knee arthroplasty? Knee 14, 204-7

Miller MC, Berger RA, Petrella AJ, Karmas A und Rubash HE (2001): Optimizing femoral component rotation in total knee arthroplasty. Clin Orthop Relat Res 392, 3845

Müller W: Das Knie. Form, Funktion und ligamentäre Wiederherstellungschirurgie. Springer Verlag, Berlin 1982 
Nabeyama R, Matsuda S, Miura H, Mawatari T, Kawano T und Iwamoto Y (2004): The accuracy of image-guided knee replacement based on computed tomography. J Bone Joint Surg Br $\underline{86}, 366-71$

Nagamine R, Miura H, Inoue Y, Urabe K, Okamoto YNM und Iwamoto Y (1998): Reliability of the anteroposterior axis and the posterior condylar axis for determing rotational alignment of the femoral component in total knee arthroplasty. Journal of orthopaedic Science $\underline{3}, 194-98$

Nägerl H, Kubein-Meesenburg D, Cotta H und Fanghänel J (1993):

Biomechanische Prinzipien in Diarthrosen und Synarthrosen. Teil III: Mechanik des Tibiofemoralgelenkes und Rolle der Kreuzbänder. Z Orthop Ihre Grenzgeb 131, 38596

Niethard FU und Pfeil J: Orthopädie. Hippokrates Verlag, Stuttgart 1989 Noyes FR, Schipplein OD, Andriacchi TP, Saddemi SR und Weise M (1992): The anterior cruciate ligament-deficient knee with varus alignment. An analysis of gait adaptations and dynamic joint loadings. Am J Sports Med $\underline{20}$ (6), 707-16 Oberst M, Bertsch C, Würstlin S und Holz U (2003): CT-Analyse der Beinachse nach konventioneller vs. navigierter Knieendoprothese. Erste Ergebnisse einer kontrollierten, prospektiven und randomisierten Studie. Unfallchirurg 106, 941-8 Oberst M, Bertsch C, Lahm A, Würstlin S und Holz U (2006): Regression and correlation analysis of preoperative versus intraoperative assessment of axes during navigated total knee arthroplasty. Comput Aided Surg 11, 87-91

Olcott CW und Scott RD (1999): The Ranawat Award. Femoral component rotation during total knee arthroplasty. Clin Orthop Relat Res $\underline{367}$, 39-42

Paley D und Pfeil J (2000): Prinzipien der kniegelenknahen Deformitätenkorrektur. Orthopäde $\underline{29}$, 18-38

Pavone V, Boettner F, Fickert S und Sculco TP (2001): Total condylar knee arthroplasty: a long-term followup. Clin Orthop Relat Res $\underline{388}$, 18-25

Perlick L, Bäthis H, Perlick C, Lüring C, Tingart M und Grifka J (2005): Revision total knee arthroplasty: a comparison of postoperative leg alignment after computerassisted implantation versus the conventional technique. Knee Surg Sports Traumatol Arthrosc $\underline{13}, 167-73$

Peterson LF, Fitzgerald RH, Jr. und Johnson EW, Jr. (1979): Total joint arthroplasty. The knee. Mayo Clin Proc $\underline{54}, 564-9$ 
Pinskerova V, Johal P, Nakagawa S, Sosna A, Williams A, Gedroyc W und Freeman MA (2004): Does the femur roll-back with flexion? J Bone Joint Surg Br $\underline{66}$, 925-31

Pitto RP, Graydon AJ, Bradley L, Malak SF, Walker CG und Anderson IA (2006): Accuracy of a computer-assisted navigation system for total knee replacement. J Bone Joint Surg Br $\underline{88}, 601-5$

Poilvache PL, Insall JN, Scuderi GR und Font-Rodriguez DE (1996): Rotational landmarks and sizing of the distal femur in total knee arthroplasty. Clin Orthop Relat Res $\underline{31}$, 35-46

Restrepo C, Hozack WJ, Orozco F und Parvizi J (2008): Accuracy of femoral rotational alignment in total knee arthroplasty using computer assisted navigation. Comput Aided Surg 13, 167-72

Robertsson O, Ranstam J und Lidgren L (2006): Variation in outcome and ranking of hospitals: an analysis from the Swedish knee arthroplasty register. Acta Orthop $\underline{77}$, 487-93

Rodriguez JA, Bhende H und Ranawat CS (2001): Total condylar knee replacement: a 20-year followup study. Clin Orthop Relat Res $\underline{388}$, 10-7 Romero J, Stähelin T, Binkert C, Pfirrmann C, Hodler J und Kessler O (2007): The clinical consequences of flexion gap asymmetry in total knee arthroplasty. J Arthroplasty 22(2), 235-40

Schnabel P und Borelli S (2011): Endoprothesenregister: Höchsten Anforderungen genügen. Dtsch Arztebl 108(48), 2598-602

Schünke M, Schulte E, Schumacher U, Voll M und Wesker K: Prometheus Lernatlas der Anatomie. Allgemeine Anatomie und Bewegungssystem. 2. Auflage; Georg Thieme Verlag, Stuttgart 2007 Sharkey PF, Hozack WJ, Rothman RH, Shastri S und Jacoby SM (2002): Insall Award paper. Why are total knee arthroplasties failing today? Clin Orthop Relat Res $\underline{404}, 7-13$

Sharma L, Song J, Felson DT, Cahue S, Shamiyeh E, Dunlop DD (2001): The role of knee alignment in disease progression and functional decline in knee osteoarthritis. JAMA $\underline{286}, 188-95$

Sikorski JM (2004): Computer-assisted revision total knee replacement. J Bone Joint Surg Br $\underline{86}, 510-4$ 
Singh VK, Varkey R, Trehan R, Kamat Y, Raghavan R und Adhikari A (2012): Functional outcome after computer-assisted total knee arthroplasty using measured resection versus gap balancing techniques: a randomised controlled study. J Orthop Surg 20 (3), 344-7

Siston RA, Patel JJ, Goodman SB, Delp SL und Giori NJ (2005): The variability of femoral rotational alignment in total knee arthroplasty. J Bone Joint Surg Am $\underline{87}$, 2276-80

Smith \& Nephew GmbH Operationsanleitung (2006): PiGalileo TKR Light ${ }^{\circledR}$, Version 1.3, Operationstechnik. Smith \& Nephew GmbH, Marl 2006

Smith \& Nephew GmbH Operationsanleitung (2008): EFK®-Kniesystem.

Trikompartimentale Knieendoprothese, Operationstechnik. Smith \& Nephew GmbH, Marl 2008

Sparmann M, Wolke B, Czupalla H, Banzer D und Zink A (2003): Positioning of total knee arthroplasty with and without navigation support. A prospective, randomised study. J Bone Joint Surg Br $\underline{85}$, 830-5

Stevens JE, Mizner RL und Snyder-Mackler L (2003): Quadriceps strength and volitional activation before and after total knee arthroplasty for osteoarthritis. J Orthop Res $\underline{21}, 775-9$

Stiehl JB (2007): Computer navigation in primary total knee arthroplasty. J Knee Surg 20, 158-64

Stöckl B, Nogler M, Rosiek R, Fischer M, Krismer M und Kessler O (2004):

Navigation improves accuracy of rotational alignment in total knee arthroplasty. Clin Orthop Relat Res $\underline{426}$, 180-6

Stulberg SD, Yaffe MA und Koo SS (2006): Computer-assisted surgery versus manual total knee arthroplasty: a case-controlled study. J Bone Joint Surg Am $\underline{88}$, 47-54

Stürmer KM: Leitlinien Unfallchirurgie. 3. Auflage; Georg Thieme Verlag, Stuttgart 2001

Sun Y, Stürmer T, Günther KP und Brenner H (1997): Inzidenz und Prävalenz der Cox- und Gonarthrose in der Allgemeinbevölkerung. Z Orthop Ihre Grenzgeb $\underline{135}$, 184-92

Townley CO (1985): The anatomic total knee resurfacing arthroplasty. Clin Orthop Relat Res 192, 82-96 
Vessely MB, Whaley AL, Harmsen WS, Schleck CD und Berry DJ (2006): The Chitranjan Ranawat Award: Long-term survivorship and failure modes of 1000 cemented condylar total knee arthroplasties. Clin Orthop Relat Res $\underline{452}$, 28-34 Wagner M und Schabus R: Funktionelle Anatomie des Kniegelenks. Springer Verlag, Berlin 1982

Walde TA, Burgdorf D und Walde HJ (2005): Process optimization in navigated total knee arthroplasty. Orthopedics $\underline{28}, 1255-8$

Walde TA, Bussert J, Sehmisch S, Balcarek P, Stürmer KM, Walde HJ und Frosch KH (2010): Optimized functional femoral rotation in navigated total knee arthroplasty considering ligament tension. Knee 17(6), 381-6 Wasielewski RC, Galante JO, Leighty RM, Natarajan RN und Rosenberg AG (1994): Wear patterns on retrieved polyethylene tibial inserts and their relationship to technical considerations during total knee arthroplasty. Clin Orthop Relat Res $\underline{299}$, 31-43

Weseloh G und Swoboda B: Arthrose; in: Rheumaorthopädie; hrsg. v. Assoziation für Orthopädische Rheumatologie; Steinkopff Verlag, Darmstadt 2005

Whiteside LA: Ligament Balancing - Weichteilmanagement in der Knieendoprothetik. 1. Auflage; Springer-Verlag, Berlin 2004

Whiteside LA und Arima J (1995): The anteroposterior axis for femoral rotational alignment in valgus total knee arthroplasty. Clin Orthop Relat Res $\underline{321}$, 168-72 Wirth CJ, Zichner L und Kohn D: Orthopädie und Orthopädische Chirurgie - Knie. 1. Auflage; Georg Thieme Verlag, Stuttgart 2005

Yau WP, Chiu KY, Zuo JL, Tang WM und Ng TP (2008): Computer navigation did not improve alignment in a lower-volume total knee practice. Clin Orthop Relat Res $\underline{466}(4), 935-45$ 


\section{Anhang}

\subsection{Abbildungsverzeichnis}

Abbildung 1: Anatomie des gesunden menschlichen Kniegelenks .................................................. 5

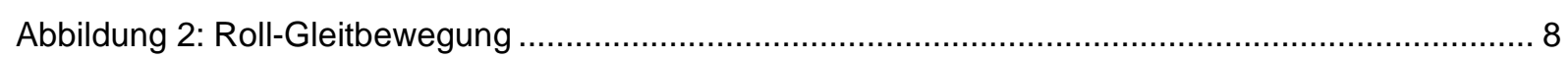

Abbildung 3: Schematische Darstellung der wichtigsten Beinachsen und Winkel in Extension ........... 10

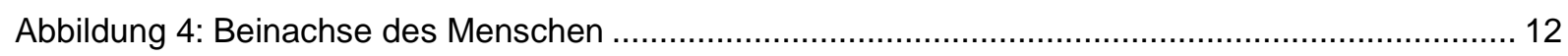

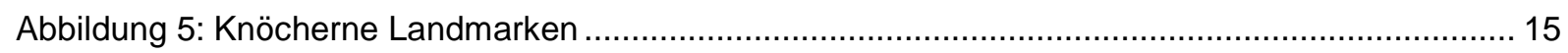

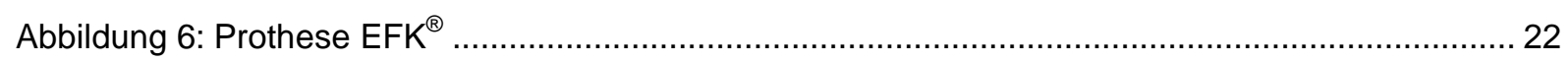

Abbildung 7:Schematische Übersicht der Achs- und Winkelverhältnisse ....................................... 23

Abbildung 8: Navigationssystem PiGalileo TKR Light ${ }^{\circledR}$, Version 1.3............................................... 25

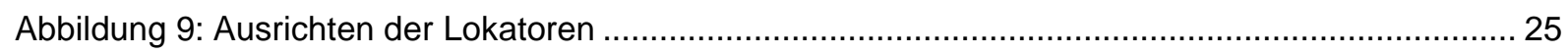

Abbildung 10: In-vivo-Aufnahme bei flektiertem Kniegelenk zur Bestimmung der mechanischen

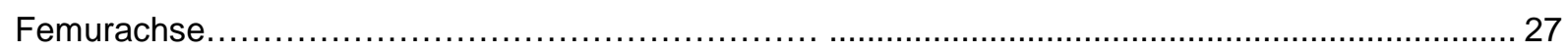

Abbildung 11: Monitorbild - Durchstoßpunkt der mechanischen Achse am distalen Femur................ 27

Abbildung 12: In-vivo-Aufnahme bei Bestimmung der Whiteside-Linie ............................................. 28

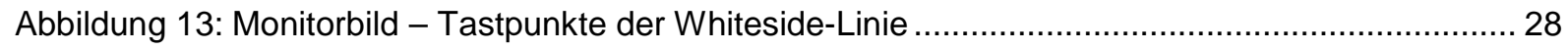

Abbildung 14: Monitorbild - proximale mechanische Achse der Tibia............................................ 29

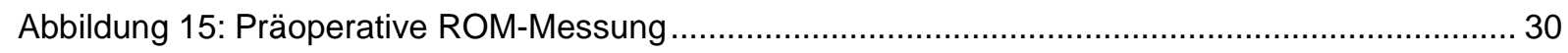

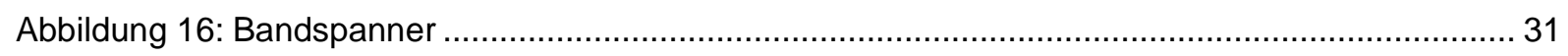

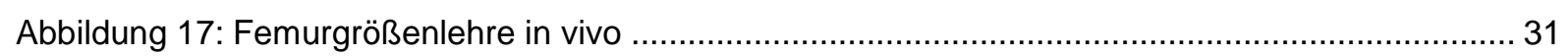

Abbildung 18: Navigationsunterstützte bandspannungsbasierte Festlegung der distalen femoralen

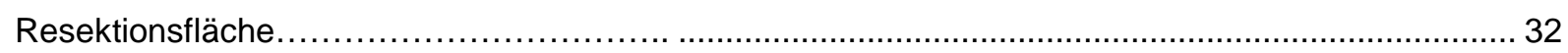

Abbildung 19: Positionierung des 4-in-1-Sägeblocks (speed block) in vivo ...................................... 32

Abbildung 20: Monitorbild - Wahl des PE-Inlays und Messung des Resektionsspalts ........................ 33

Abbildung 21: Rotations- und Größenbestimmung der Femurkomponente unter Berücksichtigung der

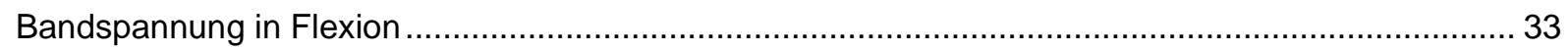

Abbildung 22: Postoperative ROM-Messung (Beispielabbildung eines Studienpatienten)................... 34

Abbildung 23: Bespielmodell einer VRML-Darstellung................................................................ 36

Abbildung 24: Beispiel der kinematischen Achsabweichung der Beinachse .................................... 37

Abbildung 25: ROM-Messung im Vergleich der jeweiligen femoralen Rotationsausrichtung ............... 38

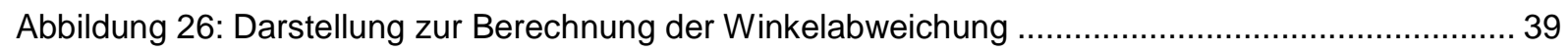

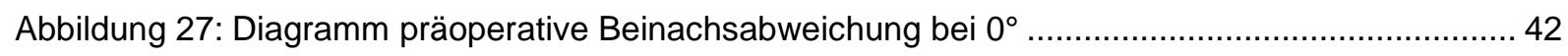

Abbildung 28: Boxplot mit Darstellung der Kurvenfläche ............................................................. 44

Abbildung 29: Korrelation zwischen Kurvenfläche und Winkelabweichung ….................................. 45

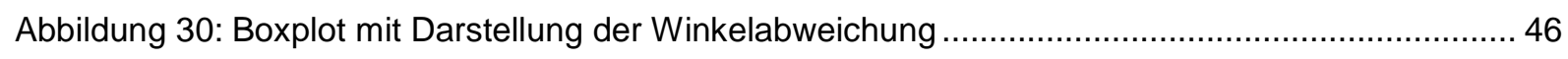

Abbildung 31: Vergleich der mittleren Winkelabweichungen aus den Funktionsdiagrammen ............. 47

Abbildung 32: Boxplot zur Darstellung der Beinachsabweichung bei $90^{\circ}$-Flexion.............................. 49

Abbildung 33: Vergleich absolute Abweichung dynamische Beinachse bei $90^{\circ}$-Flexion ..................... 49

Abbildung 34: Boxplot mit Abweichung der Beinachse in Streckstellung ……................................. 50 
Abbildung 35: Diagramm postoperative Beinachsabweichung bei $0^{\circ}$

Abbildung 36: Boxplot zur Darstellung der Rotationsabweichung beim Beugespalt 53

Abbildung 37: Vergleich Mittelwerte der absoluten Winkel der femoralen Rotationsausrichtungen zur tibialen Resektionsfläche beim Beugespalt.

\section{Bemerkung:}

Die intraoperativen Fotographien wurden mit einer Nikon ${ }^{\circledR}$ Coolpix $L 11$ angefertigt. Die Anfertigung der eigenen Abbildungen erfolgte mit Adobe ${ }^{\circledR}$ Photoshop $^{\circledR} 7.0$. 


\subsection{Tabellenverzeichnis}

Tabelle 1: Radiologische Einteilung der Arthrose nach Kellgren und Lawrence ................................ 13

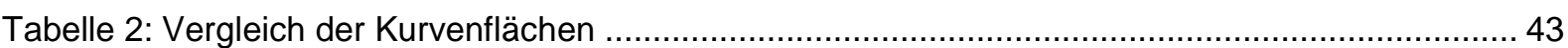

Tabelle 3: Vergleich der Funktionsdiagramme durch Bestimmung der Winkelabweichung .................. 45

Tabelle 4: Dynamische Beinachsabweichungen bei $90^{\circ}$-Flexion...................................................... 48

Tabelle 5: Femorale Rotationsabweichung zur tibialen Resektionsfläche bei $90^{\circ}$-Flexion................... 52 


\section{Danksagung}

Meinen besonderen Dank möchte ich Herrn Dr. med. Hans-Joachim Walde für die Promotionsmöglichkeit in seiner Abteilung sowie für seine überaus freundliche, kompetente und hilfsbereite Unterstützung bei der Durchführung meiner Studie aussprechen.

Zusätzlich möchte ich seinem Sohn Herrn PD Dr. med. Tim Alexander Walde für die Betreuung meiner Dissertation und die effiziente Zusammenarbeit sowie die konstruktiven Verbesserungsvorschläge ganz herzlich danken.

Für die Annahme und Betreuung der Dissertation möchte ich mich bei Herrn Prof. Dr. med. Karl-Heinz Frosch sehr bedanken.

Ich möchte mich auch bei Herrn Christian Tepe von AXIOS 3D ${ }^{\circledR}$ Services $\mathrm{GmbH}$ für die EDV-basierte Realisierung meiner Konzeption bedanken.

Letztendlich gehört ein besonderer Dank auch meinen Eltern und meiner Frau für ihre moralische Unterstützung. 